University of Louisville

ThinkIR: The University of Louisville's Institutional Repository

Electronic Theses and Dissertations

7-2011

\title{
Feasibility of subcutaneous ECG leads for synchronized timing of a counterpulsation device.
}

Sean P. Warren

University of Louisville

Follow this and additional works at: https://ir.library.louisville.edu/etd

\section{Recommended Citation}

Warren, Sean P., "Feasibility of subcutaneous ECG leads for synchronized timing of a counterpulsation device." (2011). Electronic Theses and Dissertations. Paper 1533.

https://doi.org/10.18297/etd/1533

This Master's Thesis is brought to you for free and open access by ThinkIR: The University of Louisville's Institutional Repository. It has been accepted for inclusion in Electronic Theses and Dissertations by an authorized administrator of ThinkIR: The University of Louisville's Institutional Repository. This title appears here courtesy of the author, who has retained all other copyrights. For more information, please contact thinkir@louisville.edu. 


\title{
FEASIBILITY OF SUBCUTANEOUS ECG LEADS FOR SYNCHRONIZED TIMING OF A COUNTERPULSATION DEVICE
}

\author{
By \\ Sean P. Warren, B.S. \\ University of Louisville, 2010

\begin{abstract}
A Thesis
Submitted to the Faculty of the Speed School of Engineering of the University of Louisville As Partial Fulfillment of the Requirements

For the Professional Degree of
\end{abstract}

\section{MASTER OF ENGINEERING}

Department of Bioengineering

University of Louisville

Louisville, Kentucky

July 29, 2011 


\section{FEASIBILITY OF SUBCUTANEOUS ECG LEADS FOR SYNCHRONIZED TIMING OF A COUNTERPULSATION DEVICE}

By

Sean P. Warren, B.S.

University of Louisville, 2010

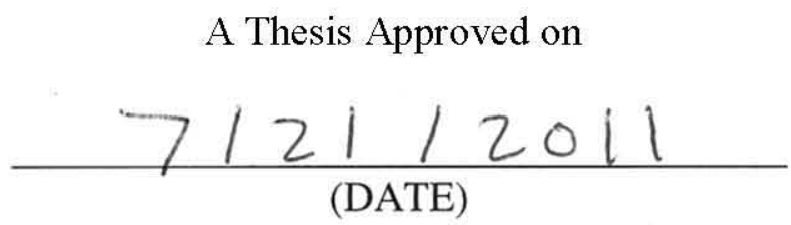

By the following Thesis Committee

Steven C. Koenig, PhD, Thesis Director

Guruprasad A. Giridharan,, PhD - Thesis Advisor

Robert S. Keynton, $\mathrm{PhD}$ - Thesis Committee

William D. Ehringer, PhD - Thesis Committee 


\section{DEDICATION}

This thesis is dedicated to my fiancée,

Rachael E. Rice. 


\section{ACKNOWLEDGMENTS}

This study was funded, in part, by NIH SBIR phase I grant (1R43HL102981-01). I would like to acknowledge and thank Dr. Paul Spence, Dr. Robert Dowling, and Daiga Koenig for allowing me the opportunity to work with them at SCR Inc. on this thesis topic. You all have opened so many doors for me, and I wish you the best of luck with your projects in the future. I would also like to thank Dr. Steven Koenig and Dr. Guruprasad Giridharan for being my mentors on this project, and for being a constant source of help and advice throughout my Bioengineering studies. I would like to thank Dr. Keynton for his guidance throughout my undergraduate career and for all of the opportunities that he has provided me. I want to thank Scott Berry for giving me a taste of graduate research and $\mathrm{PhD}$ Comics. I would like to thank the students and staff of the Cardiovascular Innovations Institute, among whom Cary Woolard, Mary Anne Hauck, and Laura and Karen Lott provided immense help. I would like to also thank Landon Tompkins for his help running the Matlab code - you will never get those countless hours back. I would like to thank my parents who have always been there for me; I hope I've made you proud! Lastly, I would like to thank my wonderful fiancée Rachael Rice. Without your love and support, I wouldn't have made it through Speed School! 


\begin{abstract}
Background: Emerging circulatory support devices that operate in counterpulsation to the native heart require synchronized timing of device ejection and filling on a beat-tobeat basis with the native heart using a patient's ECG. Surface leads are commonly used for short-term patient monitoring but not appropriate for long-term use, and epicardial and non-thoracotomy leads increase the complexity of the device implant/explant procedures. Subcutaneous leads have been shown to be less susceptible to artifacts than surface leads, require less invasive surgery, and have recently been used successfully with a long-term subcutaneous implantable cardioverter-defibrillator. The objective of this study was to develop subcutaneous ECG leads for synchronized timing (filling and ejection cycles) of the Symphony device and wearable pneumatic driver for chronic counterpulsation therapy. To demonstrate feasibility, we tested the hypothesis that subcutaneous ECG leads provide equivalent QRS detection, lead migration, and durability as clinical-standard epicardial leads.
\end{abstract}

Methods: Two epicardial and six subcutaneous leads were implanted in bovine (7 days, $\mathrm{n}=4$ and 14 days, $\mathrm{n}=2$ ). Epicardial and subcutaneous ECGs were recorded simultaneously in 30 second epochs every hour and in 20 minute epochs during daily treadmill exercise. Landmark features and R-wave triggering detection rates for each lead configuration were calculated and compared. Lead placement, migration, and durability were quantified using fluoroscopy and evaluated at necropsy. 
Results: There were 2,818 data epochs recorded at rest and 24 data epochs recorded during treadmill exercise. Using a simple adaptive-thresholding $\mathrm{R}$-wave detection algorithm with the epicardial signal as the control, the triggering rate for the subcutaneous ECG leads demonstrated $99.1 \pm 0.4 \%$ positive predictive value and $96.8 \pm 1.5 \%$ sensitivity during normal daily activity, and $98.0 \pm 10.2 \%$ positive predictive value and $93.3 \pm 5.6 \%$ sensitivity during treadmill exercise. The average QRS voltage was $818 \pm 99 \mu \mathrm{V}$ with a $\mathrm{T} / \mathrm{R}$ ratio of $0.44 \pm 0.05$. There was no significant waveform distortion or artifacts observed in ECG waveforms measured with the subcutaneous leads. Upon study endpoint, all subcutaneous leads (36/36) were within $1.4 \mathrm{~cm}$ of initial placement with an average migration distance of $0.52 \pm 0.10 \mathrm{~cm}$. There was no lead fracture or permanent signal loss for all leads (36/36).

Conclusion: In this study, the subcutaneous leads provided equivalent performance to epicardial leads in bovine during normal daily activity and treadmill exercise. The leads were easily implantable using standard surgical instruments, provided high reliability of QRS detection, and showed no significant migration from the initial placement site. These findings demonstrate the feasibility of using subcutaneous leads for synchronized timing of mechanical circulatory support while offering the advantage of less invasive surgery and associated risk factors. 


\section{TABLE OF CONTENTS}

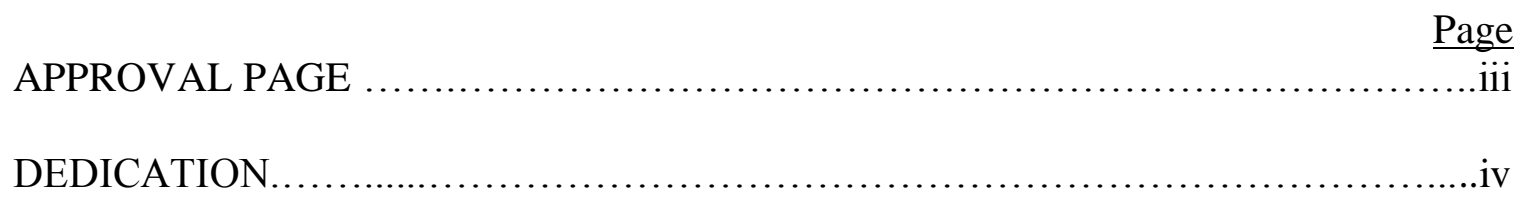

ACKNOWLEDGEMENTS..........................................................

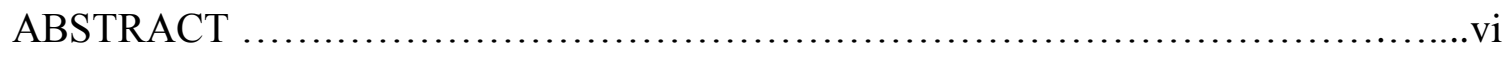

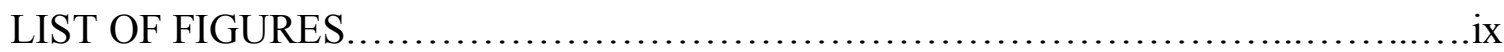

I. BACKGROUND.................................................

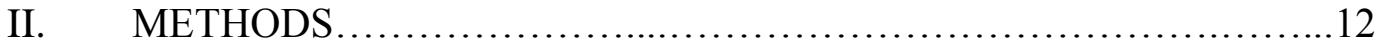

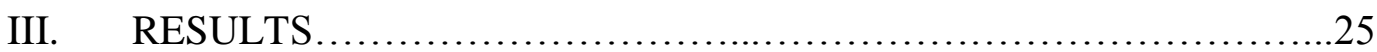

IV. DISCUSSION, LIMITATIONS AND CONCLUSION...................30

V. WORKS CITED .................................................

VI. CURRICULUM VITAE .......................................... 37

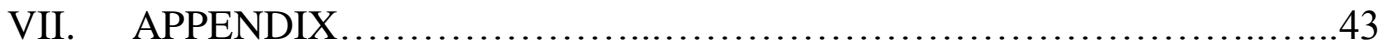




\section{LIST OF FIGURES}

Figure 1. The Symphony is implanted in the patient's right side via a pacemaker-like pocket, anastomosed to the right axillary artery, and the driveline exits through the skin (left). The pump (center) fills during systole and empties during diastole through a valveless, silicon-coated ePTFE cannula coated. The pump is actuated by a small,

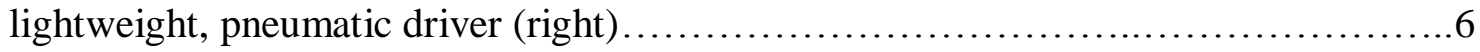

Figure 2. The 32-ml Symphony provided a greater reduction in left ventricular myocardial oxygen consumption $\left(\mathrm{LV} \mathrm{O}_{2}\right)$ and a greater increase in mean diastolic coronary artery flow (CAFd) per unit left ventricular external work (LVEW). These data demonstrate the 32-ml Symphony improves myocardial perfusion (supply) more than a

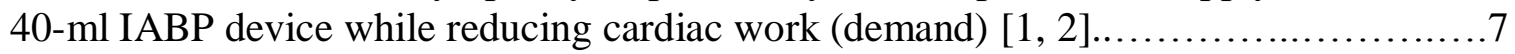

Figure 3. Measured surface voltages during ventricular depolarization show that the best change in voltage can be achieved with vertical lead pairs across the right chest (Left). An analog lead configuration was tested in a calf model (Right) .................... 10

Figure 4: Intracardiac, subcutaneous, and surface ECG leads during acute ECG mapping study conducted on 6-2-09 (1-left) and 6-25-09 (2-right)........................ 10

Figure 5: A comparison of intracardiac, subcutaneous, and surface ECG recordings during acute animal ECG mapping experiments conducted on 6-24-09 (1, left) and 6-2509 (2, right). The subcutaneous and surface ECG signals show comparable fidelity to the intracardiac ECG waveform................................................ 11

Figure 6. Illustration of experimental protocol for testing and comparison of subcutaneous and epicardial leads. ECG waveforms were recorded simultaneously in bovine during normal daily activity and treadmill exercise......................... 12

Figure 7. Subcutaneous ECG lead with extended sensing wire. Enlarged view shows the coiled sensing wire. ....................................................... 13

Figure 8. Ventral-dorsal lead pair configurations on the right side of the calf. All leads

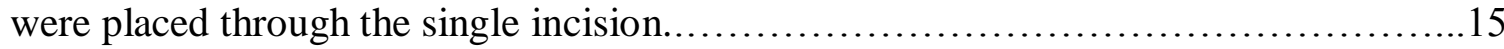

Figure 9. Summary of post-processing analysis of subcutaneous and epicardial ECG waveforms recorded in calves during normal activity and treadmill exercise. After the QRS identification, the R-R interval was calculated, allowing for time-based isolation of

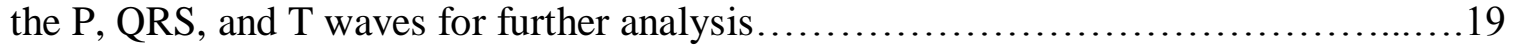

Figure 10. Average FFT of a subcutaneous lead pair over seven days..................20

Figure 11. Sample ECG waveforms measured with epicardial (top) and subcutaneous (bottom) leads plotted over the stem plot of the detection matrix. The vertical thick line indicates a detected QRS complex. 
Figure 12. Subcutaneous versus epicardial ECG signals for both normal calf activity (left)

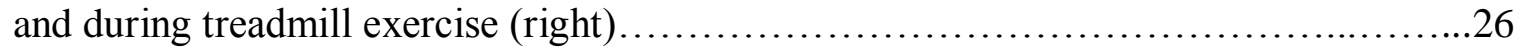

Table 1. Positive Predictive Value (PPV), Sensitivity, and ECG landmark voltages for all ECG lead pairs at rest and during treadmill exercise..............................27

Figure 13. Fluoroscopic digital image of implanted ECG subcutaneous leads at 14-days post-implant. Staples were placed on the skin at time of implant to mark placement of the subcutaneous leads (left). Image reveals no significant migration of the subcutaneous leads (right), which was quantified using ImageJ (NIH, Bethesda MD) analysis

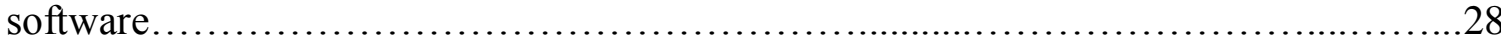




\section{CHAPTER I - BACKGROUND}

\section{Heart Failure}

Heart failure (HF) is one of the largest unsolved problems in cardiac care today. There are more than 5.8 million patients in the US alone with this diagnosis and the number of patients is expected to double over the next ten years. Among the three major forms of cardiovascular disease (HF, coronary artery disease and stroke), only HF has shown a significant increase in hospitalization rates. Between 1980 and 2006, the number of patients over age 65 that were hospitalized for HF increased 131\% [3]. In 2008, the direct and indirect costs of HF in the US were estimated to be 34.8 billion dollars [4]. Globally, the incidence of $\mathrm{HF}$ is also increasing with over one million new cases diagnosed annually. Current treatment options for HF include pharmacological therapy, cardiac resynchronization therapy (CRT), mechanical heart assist, and heart transplantation. Despite improvements in survival with medical therapy and CRT, the prognosis with heart failure remains poor. In a recent longitudinal study, mortality at one year was $15.0 \%$ and $28.0 \%$ respectively for NYHA Class III and IV [5]. Two-year mortality in the RALES trial was approximately 30\% [6]. In advanced stages of HF (NYHA Class IV), heart transplantation offers the best opportunity for long-term survival. However, heart transplantation is restricted to select patients based on multiple factors including age, prior operations, end-organ function, and even appropriate insurance coverage. Furthermore, the number of available donor organs $(\sim 2,300 / \mathrm{yr}$ in the US according to the Organ Procurement and Transplantation Network as of 2011) cannot meet the growing demand. The shortage of appropriate donor hearts is worse in other countries such as Canada (<200 heart donors/year) and Japan (11 heart transplants in 2009) [7, 8]. 
The large patient population with advanced HF and the limited number of donor organs has stimulated the development of mechanical assist devices both as a bridge to transplant and as permanent support devices (destination therapy). Despite years of research and hundreds of millions of dollars in expenditure, only approximately 4,000 patients receive mechanical circulatory support each year. Current mechanical circulatory support devices require a major surgical procedure (sternotomy or thoracotomy) and the use of cardiopulmonary bypass for implantation. Further, postoperative management of these patients can be challenging and costly. Due to the complexity of the devices and the major surgical procedure required for implantation, complications are not uncommon and include device failure, thromboembolism, stroke, infection, and bleeding. For these reasons, long term mechanical support is reserved for patients with advanced heart failure who are unlikely to recover ventricular function. Additionally, the high cost of these devices, over $\$ 67,000$ for the left ventricular assist devices (LVADs) alone [9], has limited adoption in large, global markets such as India and China and has contributed to devices not being approved for long-term use by government regulatory agencies (UK) [10]. The NHLBI has recognized the need for innovative approaches to the treatment of HF. In 2003, the NHLBI "Recovery from Heart Failure with Circulatory Assist" working group recommended the development of novel therapies for myocardial recovery, and elucidation of the mechanisms leading to reverse remodeling and myocardial recovery [11]. 


\section{Counterpulsation Therapy}

Introduced in the 1960 's, counterpulsation is considered a proven method of providing mechanical circulatory support. Basically, counterpulsation is the removal or displacement of arterial blood during ventricular systole to provide afterload reduction and "return" of the blood during diastole to augment coronary and systemic perfusion. Counterpulsation has many important clinical benefits for the heart, end organs and the peripheral circulation for patients with moderate myocardial dysfunction. Counterpulsation therapy increases the diastolic aortic pressure by $30-70 \%$ improving end-organ perfusion and coronary perfusion [12-14]. The ejection pressure of the native ventricle is diminished, reducing afterload and left ventricular external work [12, 15-18]. The peak systolic pressure is reduced by up to $15 \%$ [12, 16, 17], while the end diastolic pressure is decreased by up to $30 \%[12,16,17]$. Cardiac output and stroke volume have been found to increase by up to $20 \%[13,19]$. Counterpulsation therapy also decreases the native heart rate by $10 \%$ [15], and may reduce the left ventricular end diastolic volume by $10-15 \%$ [20]. The hemodynamic benefits of counterpulsation support also translate into improved metabolic function and often assist in recovery of end organ function [15]. Counterpulsation therapy improves the cardiac energy balance by increasing the oxygen supply to the myocardium through increased coronary perfusion $[21,22]$ and by reducing myocardial oxygen consumption through a decrease in afterload and left ventricular work[15]. Counterpulsation therapy has been shown to augment cerebral, renal, mesenteric and pulmonary blood flows [23-25]. Increases in urine output, decreases in lactic acidosis and enhancement of venous oxygen saturation have also been observed in patients [15], demonstrating improved end organ function. 
Counterpulsation is currently achieved with placement of an intra-aortic balloon pump (IABP), which is used clinically for limited time periods of several hours to several days. Each year more than 160,000 patients worldwide receive IABP therapy and, as a result, clinicians have developed a great deal of confidence in the therapeutic effectiveness of IABP counterpulsation $[12,15]$. Placement of an IABP simply requires percutaneous access to the femoral artery. While the percutaneous access used for IABPs is less invasive than with LVADs, complication rates increase with duration of IABP support, and patients are unable to ambulate. These issues along with the limited durability of the balloon itself preclude the use of IABP for chronic support.

Given the proven benefits of counterpulsation, it is not surprising that a number of investigators have tried to develop devices that can provide chronic counterpulsation (LVAD Technologies, and Sunshine Medical). Jeevanandam et al. reported on clinical outcomes of a counterpulsation device (CardioPlus, LVAD Technologies) that was sewn to the descending aorta [26]. After implantation, these patients had significantly increased cardiac output and significantly decreased right atrial and pulmonary capillary wedge pressures. Improved cardiac output and end organ perfusion were also evidenced by a significant improvement in serum creatinine. Additionally, there was a significant decrease (improvement) in NYHA functional class [26]. The CardioPlus did not achieve clinical acceptance due to the need to perform a full thoracotomy and the use of cardiopulmonary bypass to place the device. However, this trial clearly demonstrated the hemodynamic and clinical benefits, and the potential for improved native heart function with chronic counterpulsation in patients with advanced heart failure. The Sunshine Heart 
device is a counterpulsation device that is wrapped around the ascending aorta and therefore requires a thoracotomy or a sternotomy for placement. The presence of previous heart surgeries, and particularly the presence of coronary artery bypass grafts, will limit or preclude the use of this device. Also, placement of this device may significantly complicate further therapies such as LVAD placement or heart transplantation. The Sunshine Heart trial is currently enrolling patients in an IDE trial. Preliminary data suggests significant hemodynamic benefits [27].

\section{Symphony}

"Symphony" is the name given by Abiomed in late 2009 to a counterpulsation device developed in conjunction with SCR Inc (Louisville, KY) and the University of Louisville. The Symphony device (currently in pre-clinical trials) is a $30-\mathrm{mL}$ stroke volume polyurethane-lined pumping chamber, which is designed to fit comfortably in a pacemaker-like pocket on the right side of a patient $[2,28]$. The pumping chamber is connected to the systemic circulation by a short, silicon-coated ePTFE graft anastomosed to the right axillary artery using a simple surgical procedure. A percutaneous driveline runs from the pumping chamber, exits the skin near the costal margin, and connects to a small pneumatic external driver. During systole, the driver evacuates air from the pumping chamber, thus removing blood from the circulation and reducing cardiac work. During diastole, the Symphony ejects the blood into the circulation providing diastolic augmentation and improving coronary perfusion. 

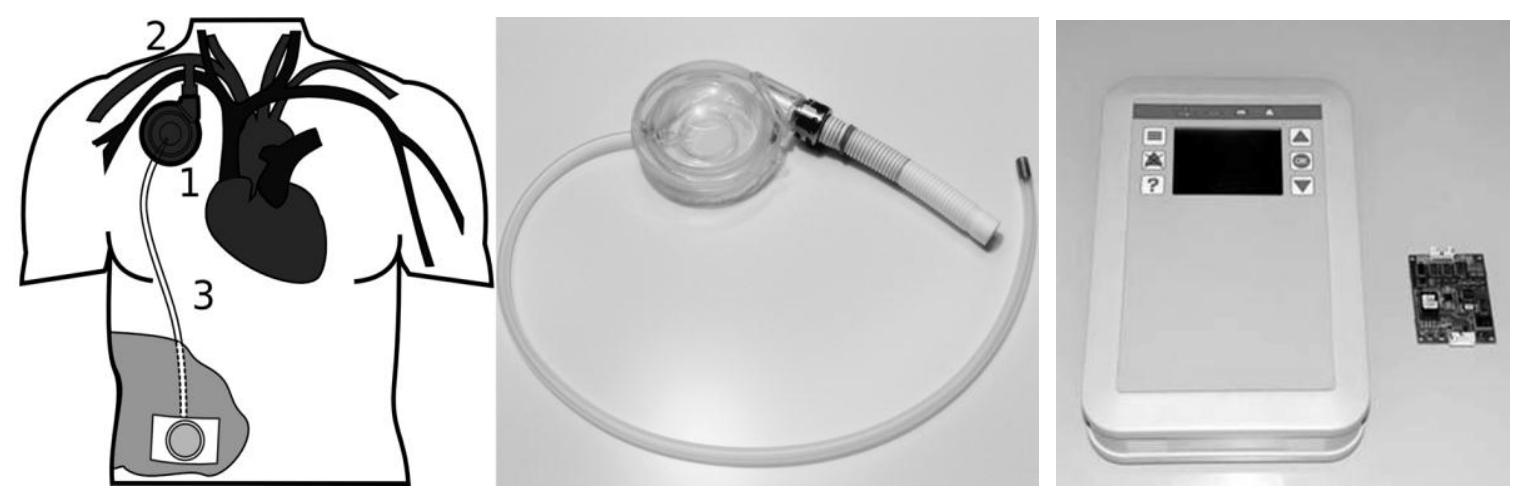

Figure 1. The Symphony is implanted in the patient's right side via a pacemaker-like pocket, anastomosed to the right axillary artery, and the driveline exits through the skin (left). The pump (center) fills during systole and empties during diastole through a valveless, silicon-coated ePTFE cannula coated. The pump is actuated by a small, lightweight, pneumatic driver (right).

Investigators have demonstrated, in computer simulations, in vitro and multiple in vivo experiments, that the Symphony device is as effective or better than an IABP (Figure 2)

$[1,2]$. Placement of the Symphony device is achieved through a simple infraclavicular incision similar to that used for placement of a pacemaker. The device itself is placed superficial to the pectoralis muscle (i.e. in the subcutaneous tissue). With this operative approach, Symphony gains the proven hemodynamic benefits of chronic counterpulsation while avoiding the surgical trauma associated with a thoracotomy or a sternotomy. Symphony may be uniquely suitable to deliver counterpulsation therapy to patients that have earlier stage HF in order to improve functional capacity, restore quality of life, allow for improved medical therapy, and promote myocardial recovery. 

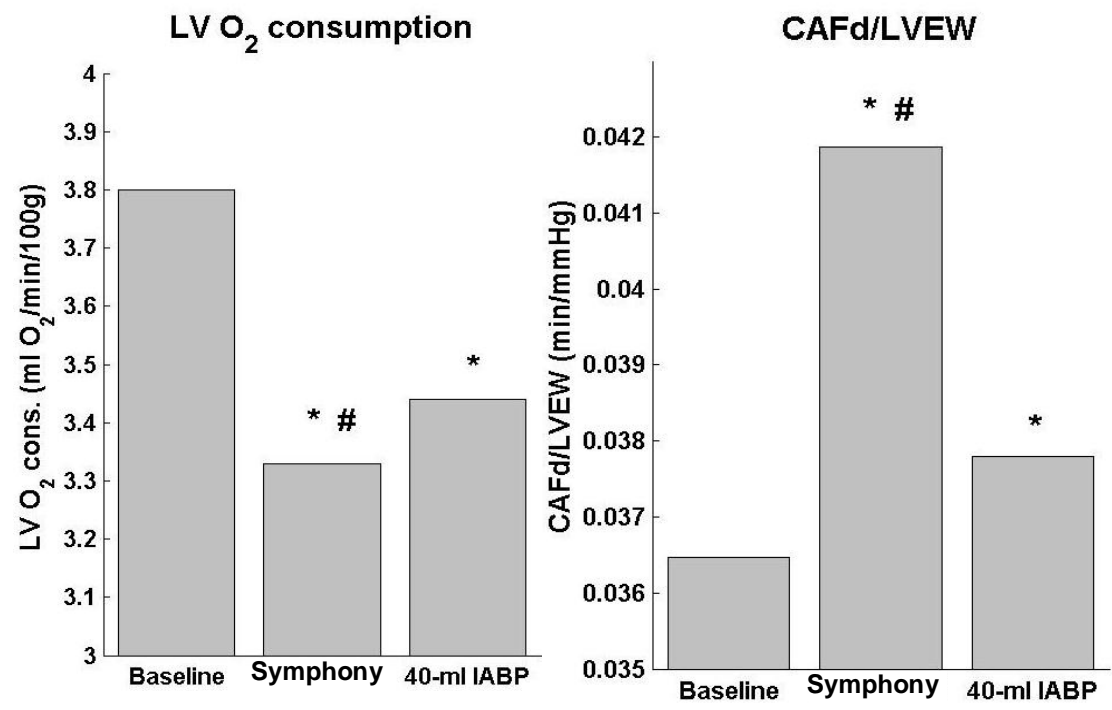

Figure 2. The 32-ml Symphony provided a greater reduction in left ventricular myocardial oxygen consumption $\left(\begin{array}{lll}\mathrm{LV} & \mathrm{0}_{2}\end{array}\right)$ and a greater increase in mean diastolic coronary artery flow (CAFd) per unit left ventricular external work (LVEW). These data demonstrate the 32-ml Symphony improves myocardial perfusion (supply) more than a 40-ml IABP device while reducing cardiac work (demand) [1, 2].

\section{ECG Acquisition and the Clinical Need for Subcutaneous Leads}

Counterpulsation devices require precise synchronized timing of device ejection and filling on a beat-to-beat basis with the native heart using a patient's ECG. Specifically, effectiveness of counterpulsation therapy is due to the synchronous timing of the ejection and filling of the circulatory support device with the filling and ejection of the native ventricles to reduce left ventricular external work and increase coronary perfusion. If the actions of the device are not appropriately timed dangerous hemodynamic conditions can result. For example, early device ejection causes the premature closure of the aortic valve, decreasing stroke volume and cardiac output.

Current counterpulsation therapy utilizes an IABP to provide acute circulatory support, and the inflation and deflation of this device is timed primarily by the patient's ECG 
(although aortic pressure can be used as an alternative [29]). The 12-lead surface electrocardiogram remains the current gold standard for short-term device timing, patient monitoring and diagnosis. While technologies are being developed for chronic monitoring [30], difficulties associated with the skin-electrode interface including poor contact, skin movement, and patient wire management currently preclude surface ECGs for long-term use. Non-thoracotomy lead systems, specifically transvenous right ventricular leads, are frequently used in cardiac pacing and defibrillation devices and provide quality ECG signals. Unfortunately, the implantation of these leads has a significant learning curve and increases the complexity of counterpulsation device implant/explant procedures by requiring the use of fluoroscopy [31]. Postoperative complications with these lead systems have also been reported and include lead dislodgment, fracture, vein subclavian vein thrombosis, sensing abnormalities, and system infection requiring full explantation [32, 33].

Recently, subcutaneous lead systems (a term broadly used to indicate lead positioning in the space external to the pericardium but below the dermis) have been developed for use in cardiac rhythm monitors [34-36]. Subcutaneous ECGs have long been theorized to be an adequate surrogate for surface signals [37] and QRS detection [38], and have recently been shown to be less susceptible to motion artifacts than surface leads [39]. In relation to the Symphony device, subcutaneous leads provide the benefit of minimally invasive surgery for implantation. Furthermore, when integrated with a device, they can remain fully implanted in the body - ideal for chronic counterpulsation therapy. Nevertheless, 
implantable loop recorders with subcutaneous lead systems have had difficulties with isolation and detection of the QRS complex [40].

\section{Early Investigation of ECG Lead Systems}

SCR initiated preliminary studies to examine the feasibility of subcutaneous ECGs for device triggering. Preliminary subcutaneous ECG studies were first performed as an adjunct to ongoing acute Symphony implantations in male Jersey calves. The Symphony device is implanted on the right subclavian artery, which requires the ECG leads to be placed over the right chest. Ideally, leads should not cross the sternum in order to avoid issues with any future cardiac procedures that require a sternotomy. Surface voltage maps of the thorax during ventricular depolarization (ECGSIM, Radboud University medical Center, Nijmegen, The Netherlands) show that vertical lead-pairs over the right chest offer the largest change in measured voltage (Figure 3, left). Several lead-pair configurations were placed across the right chest of a calf model in order to best mimic the human analog configuration (Figure 3, right). These experiments demonstrated that the subcutaneous ECG leads were comparable to intra-cardiac leads, and the lead configuration with the highest fidelity resulted when the distance between the leads was at a maximum in a parallel orientation to the mean electrical axis of the heart. Surface ECG and subcutaneous ECG mapping tests (Figure 4, 5) were conducted to determine the ECG lead configurations with the highest fidelity data (magnitude and morphology). Based upon these early experiments, development of subcutaneous ECG leads and chronic testing in bovine were initiated to demonstrate equivalency to epicardial leads for QRS detection and integration with Symphony device and driver for long-term support. 

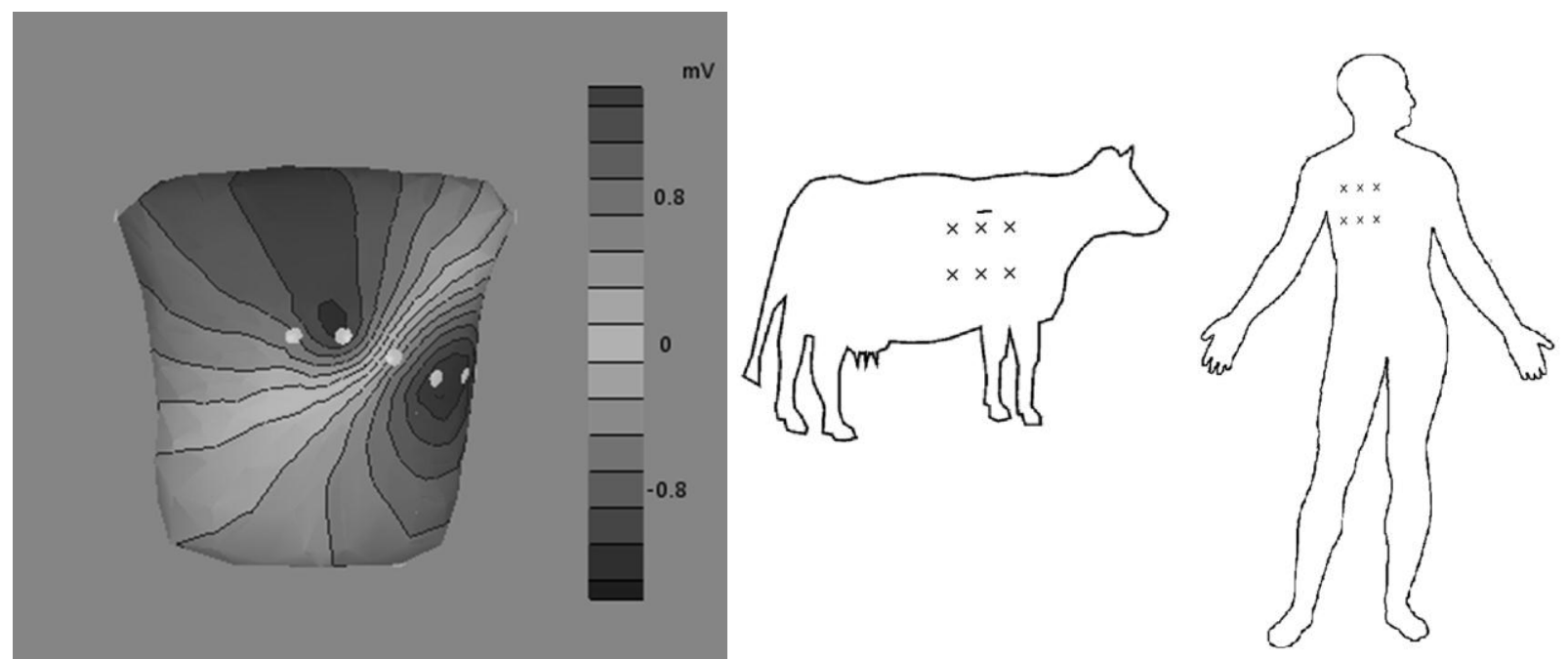

Figure 3. Measured surface voltages during ventricular depolarization show that the best change in voltage can be achieved with vertical lead pairs across the right chest (Left). An analog lead configuration was tested in a calf model (Right).
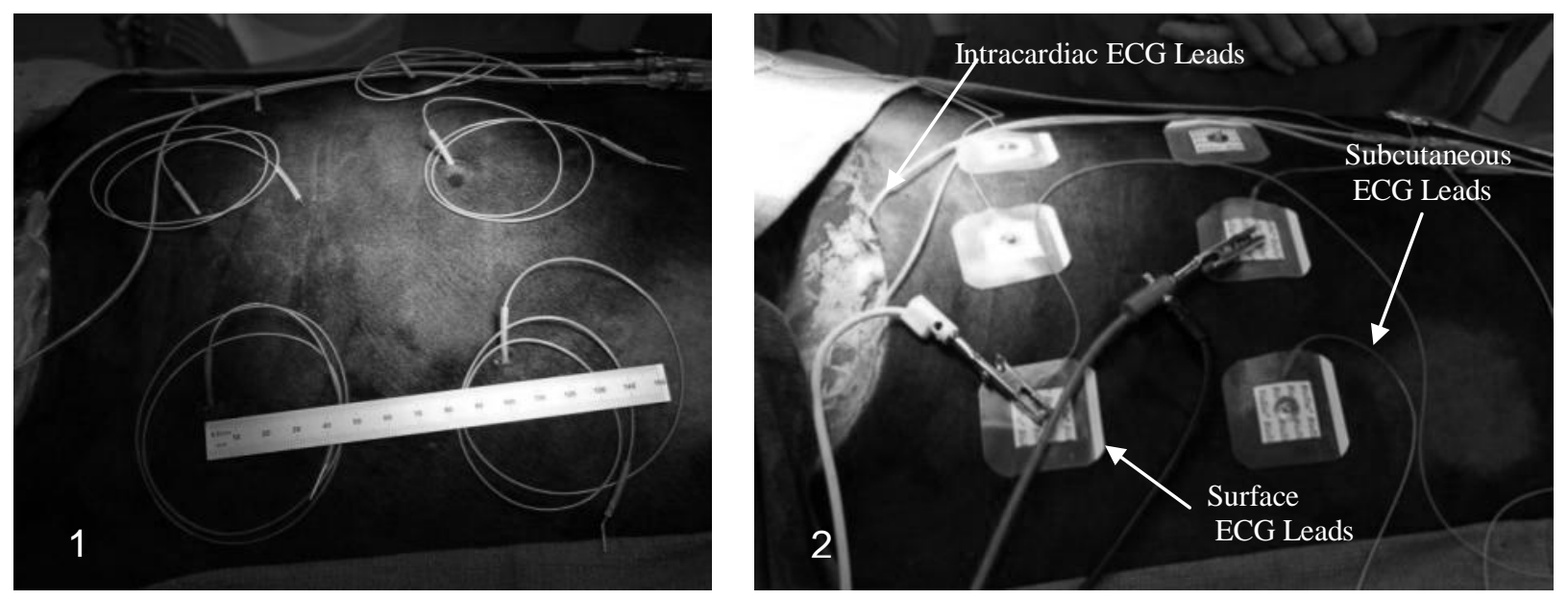

Figure 4: Intracardiac, subcutaneous, and surface ECG leads during acute ECG mapping study conducted on 6-2-09 (1-left) and 6-25-09 (2-right). 

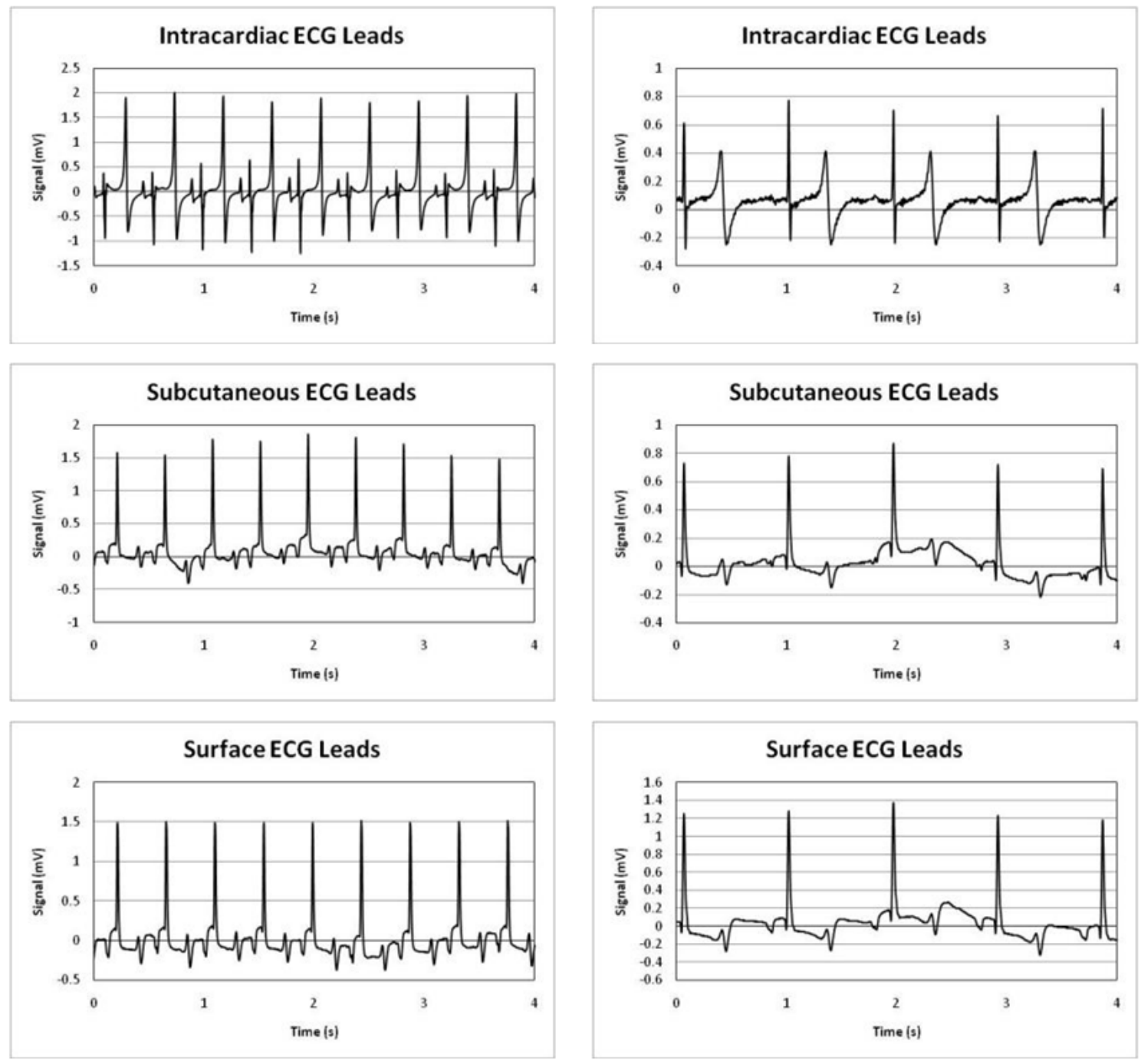

(1)

(2)

Figure 5: A comparison of intracardiac, subcutaneous, and surface ECG recordings during acute animal ECG mapping experiments conducted on 6-24-09 (1, left) and 6-25-09 (2, right). The subcutaneous and surface ECG signals show comparable fidelity to the intracardiac ECG waveform. 


\section{CHAPTER II - METHODS}

\section{Study Design and Experimental Protocol}

The objective of this study was to develop subcutaneous ECG leads for synchronized timing (filling and ejection cycles) of the Symphony device and wearable pneumatic driver for chronic counterpulsation therapy. To demonstrate feasibility, we tested the hypothesis that subcutaneous ECG leads provide equivalent QRS detection, lead migration, and durability as clinical-standard epicardial leads (Figure 6).

\section{Day Studies $(\mathrm{N}=4)$}

\begin{tabular}{|c|c|c|c|c|c|c|c|}
\hline \multicolumn{3}{|c|}{ Surgery } & Survival & \multicolumn{3}{c|}{ Endpoint } \\
\hline $\begin{array}{c}\text { SubQ } \\
\text { Leads } \\
(\mathrm{n}=6)\end{array}$ & Fluoroscopy & $\begin{array}{c}\text { Epicardial } \\
\text { Leads }\end{array}$ & $\begin{array}{c}\text { Symphony } \\
\text { System } \\
\text { Implant }\end{array}$ & $\begin{array}{c}7 \text { Days } \\
\text { ECGs recorded }\end{array}$ & $\begin{array}{c}\text { Animal } \\
\text { Euthanized }\end{array}$ & $\begin{array}{c}\text { Fluoroscopy } \\
\text { Lead Migration }\end{array}$ & $\begin{array}{c}\text { Necropsy } \\
\text { Lead Durability }\end{array}$ \\
\hline
\end{tabular}

\section{Day Studies ( $\mathrm{N}=2$ )}

\begin{tabular}{|c|c|c|c|c|c|c|c|c|}
\hline \multicolumn{3}{|c|}{ Surgery } & \multicolumn{2}{c|}{ Survival } & \multicolumn{3}{c|}{ Endpoint } \\
\hline $\begin{array}{c}\text { SubQ } \\
\text { Leads } \\
(\mathrm{n}=6)\end{array}$ & Fluoroscopy & $\begin{array}{c}\text { Epicardial } \\
\text { Leads }\end{array}$ & $\begin{array}{c}\text { Symphony } \\
\text { System } \\
\text { Implant }\end{array}$ & $\begin{array}{c}7 \text { Days with } \\
\text { ECGs recorded }\end{array}$ & $\begin{array}{c}\text { Animal } \\
\text { Treadmill } \\
\text { ECGs recorded }\end{array}$ & $\begin{array}{c}\text { Euthanized } \\
\text { Fluoroscopy } \\
\text { Lead Migration }\end{array}$ & $\begin{array}{c}\text { Necropsy } \\
\text { Lead Durability }\end{array}$ \\
\hline
\end{tabular}

Figure 6. Illustration of experimental protocol for testing and comparison of subcutaneous and epicardial leads. ECG waveforms were recorded simultaneously in bovine during normal daily activity and treadmill exercise.

In bovine, ECG was measured and recorded with subcutaneous (6 leads) and clinicalstandard epicardial (2 leads) for 7-days $(n=4)$ and 14-days $(n=2)$ during normal daily activity and treadmill exercise. Recorded ECG waveforms with subcutaneous and epicardial lead configurations were analyzed to quantify QRS detection (Matlab, The 
Mathworks, Natick MA). Fluoroscopy images (GE Innova 3100, Waukesha WI) of leads during placement (implant) were recorded, analyzed using ImageJ (NIH, Bethesda MD), and compared at necropsy (explant) to quantify lead migration. At necropsy, subcutaneous and epicardial leads were photographed and examined for any signs of fracture, damage, or infection.

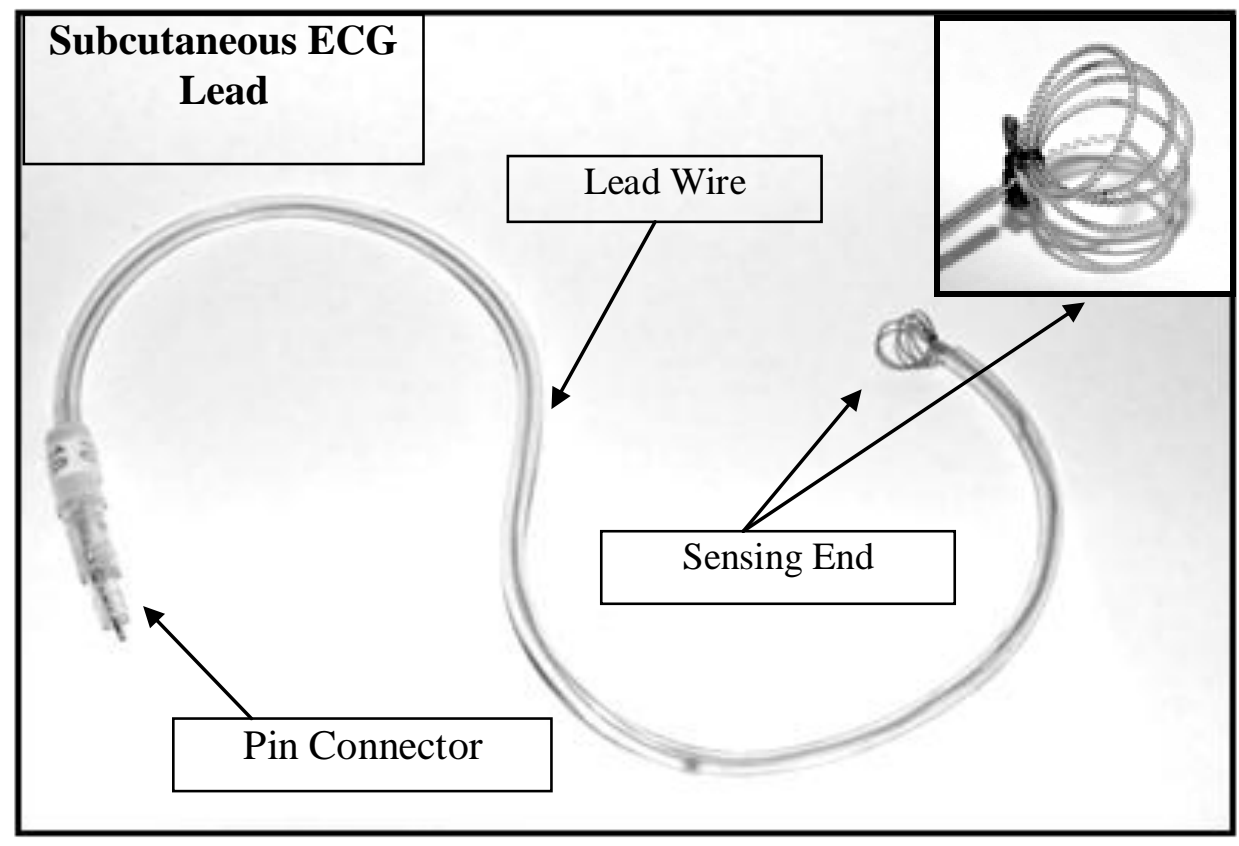

Figure 7. Subcutaneous ECG lead with extended sensing wire. Enlarged view shows the coiled sensing wire.

\section{Lead Fabrication}

The subcutaneous ECG leads were fabricated from commercially-available ECG lead coupler kits (Data Systems International, St. Paul, MN), Tygon tubing (Fischer Scientific, Pittsburg, PA), and electronic pin and socket connectors (Allied Electronics Inc., Ft. Worth, TX) (Figure 7). These leads are suitable for placement in large quadrupeds and were fabricated using a modified version of the technique described in the Data Systems 
International technical bulletin [41]. Seven centimeters of lead was exposed, looped around a clamp, and secured with a suture. Leads were sterilized using ethylene oxide. Signals were recorded using a custom data acquisition system that is in compliance with Good Laboratory Practices, as has been previously reported [42].

\section{Delivery and Placement of Subcutaneous Leads.}

Several operative approaches and devices to deliver the subcutaneous ECG leads were developed using human cadavers (Fresh Tissue Laboratory, University of Louisville), including tunneling tools, modified endoscopic clip appliers, and laparoscopic instruments. The selected method was to simply pull the lead to the desired position using a standard straight needle. A 2-0 nylon suture was passed through the loops of wire at the end of the subcutaneous leads and then through the eye of a four-inch needle. A standard needle holder was then used to control the needle. Working through the device implantation wound, the needle was advanced to the desired position on the chest and then the needle and attached suture were passed through the skin. The needle and suture were removed leaving the ECG lead in place beneath the skin. This method does not require any additional incision(s) or special training and can be accomplished with standard surgical instruments.

Preliminary studies in bovine demonstrated that a lead configuration of ventral and dorsal lead pairs produced high-quality signals (Figure 8). Specifically, robust signals were obtained when a pair of leads was at least $15 \mathrm{~cm}$ apart and within $25 \mathrm{~cm}$ of the heart. An adequate human analogue configuration is a pair of ECG leads placed $15 \mathrm{~cm}$ apart and 
parallel to the electrical axis of the heart. Similar subcutaneous configurations have been reported with implantable cardioverter-defibrillators using a parasternal electrode [34].

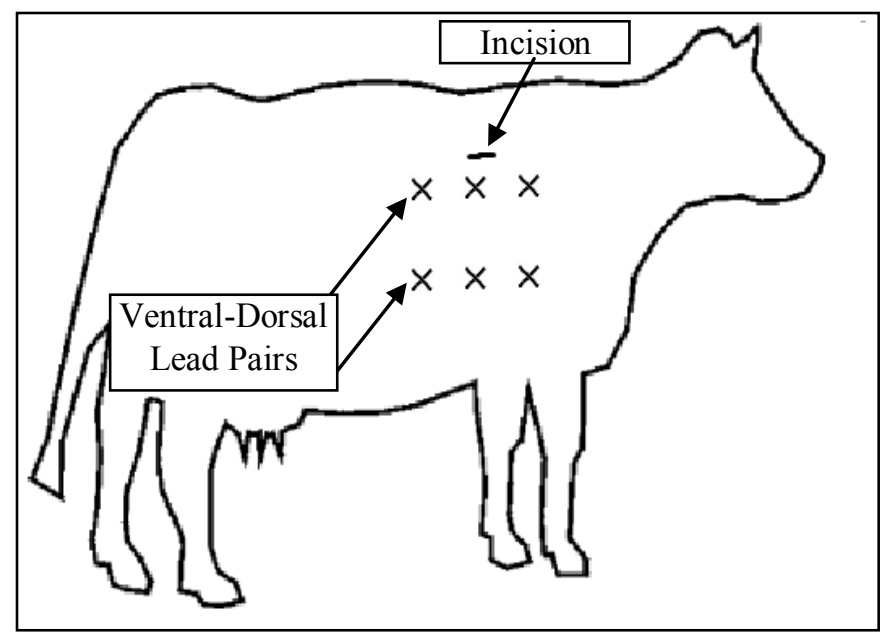

Figure 8. Ventral-dorsal lead pair configurations on the right side of the calf. All leads were placed through the single incision.

\section{Animal Study}

All animals received humane care in accordance with the Guide for Care and Use of Laboratory Animals (NIH publication 86--23, revised 1996) and the guidelines determined by the Institutional Animal Care and Use Committee (IACUC) of the University of Louisville (UofL). The study was completed in compliance with a UofL approved IACUC protocol.

The ECG study was performed concurrently with a study involving a circulatory support device implantation in a Holstein calf model $(80-100 \mathrm{~kg})$. Four animals were monitored with implanted devices for 7 days and two animals were monitored with implanted devices for 14 days. After initial physical examination and a 5-7 day quarantine period, animals were preanesthetized with Atropine $(30 \mathrm{mg})$ and then anesthetized with 
isoflurane (3\%-5\%) via nose cone. After intubation, anesthesia was maintained with isoflurane $(1 \%-3 \%)$ mixed with oxygen and room air. Animals were then prepared for surgery on the right side. Six leads were placed subcutaneously through a single incision over the right side of each animal ( $\mathrm{n}=6$ animals, 36 leads) in three ventral-dorsal pairs. The placement of all six leads was timed. Subcutaneous ECG signal was verified for each vertical lead-pair. Surgical staples were placed on the skin to mark the initial site of the subcutaneous lead. Fluoroscopy was done at the time of implant to verify that the skin staples were in immediate proximity to the subcutaneous leads.

The animal was placed on its right side and the left neck and chest were prepared and

draped. An incision was made over the fourth rib and carried down to the $4^{\text {th }}$ intercostal space. The chest was entered and a standard rib retractor was placed. The pericardium was identified and opened and two epicardial screw-in leads were placed and connected to the circulatory support device driver. Once the quality of the epicardial signal was verified the leads were tunneled transcutaneously and secured in position. The chest was closed and the circulatory support device was implanted onto the left carotid artery using previously described methods [8].

\section{Data Collection}

After the animals were recovered, the epicardial and 3 subcutaneous ECG signals were simultaneously measured and recorded with a GLP compliant data acquisition system, as previously reported [42]. Briefly, three pairs of subcutaneous leads were signal conditioned with a clinical-standard ECG amplifier (Gould Biotach 6600, Cleveland 
$\mathrm{OH}$ ). The epicardial ECG waveforms were signal conditioned by the Symphony pneumatic driver (iPulse, Abiomed, Danvers MA). All signal conditioned ECG waveforms were sampled at $400 \mathrm{~Hz}$ (National Instruments, AT-MIO-16E-10, Austin, TX), filtered (60 Hz notch, Frequency Devices, Ottawa IL), and displayed real-time on a computer monitor using custom software [43] developed with LabVIEW (National Instruments, Austin TX). Specifically, thirty-second data epochs were recorded every hour for the duration of the study. All recorded ECG waveforms were saved for postprocessing and analysis.

\section{Treadmill Testing}

For each of the 14-day animal experiments $(n=2)$, treadmill testing was initiated after the seventh postoperative day and repeated daily for the duration of the study (i.e. 7 exercise days). Calves were titrated to a walking speed of 30 meters/minute on the treadmill (Safe-T-Mill, Good Horsekeeping, Inc, Ash Grove, MO) and then maintained at that rate for up to 20 minutes or until the cow showed signs of fatigue (as evaluated and recommended by study veterinarian, Leslie Sherwood, DVM). .

\section{Lead Migration}

Subcutaneous ECG lead placement at time of Symphony device implant and explant were digitally photographed and recorded using fluoroscopy (GE Innova 3100, Waukesha WI). Surgical staples were placed at device implant as a landmark for lead placement. Commercial image processing software (ImageJ, NIH, Bethesda MD) was used to quantify lead migration by overlaying digital photographs and measuring migration 
distance. In addition, during all leads were surgical exposed, examined, and gross lead migration measured as a back-up technique.

\section{ECG Analysis (subcutaneous and epicardial)}

In analyzing the recorded subcutaneous and epicardial ECG waveforms, an algorithm was developed to meet the following design criteria: (1) use a standard QRS detection strategy, (2) robust to handle noise and artifacts, (3) handle large volumes of ECG data, and (4) feasible for integration into real-time driver software, as shown in Figure 9. Additionally, the analysis software has to allow for characterization of the subcutaneous signal by isolation of ECG landmarks.

The software code for data processing and analysis of all recorded ECG waveforms was developed in Matlab programming software (MathWorks, Natick MA). A preliminary program extracted the ECG data from the raw LabVIEW data files (*.dat), which were then passed to the SignalFiltv7 script. The code handled ECG signal alignment, signal filtering, QRS detection for the epicardial and subcutaneous signals, comparison and analysis of the two detection rates, and subcutaneous landmark isolation and characterization. It was written so that it could be run on an entire data set and save all important data into matrices that could be accessed by a later script. A description of each of the script's processes and algorithms is presented next. A complete line-by-line listing of the data processing and analysis software is presented in the Appendix. 


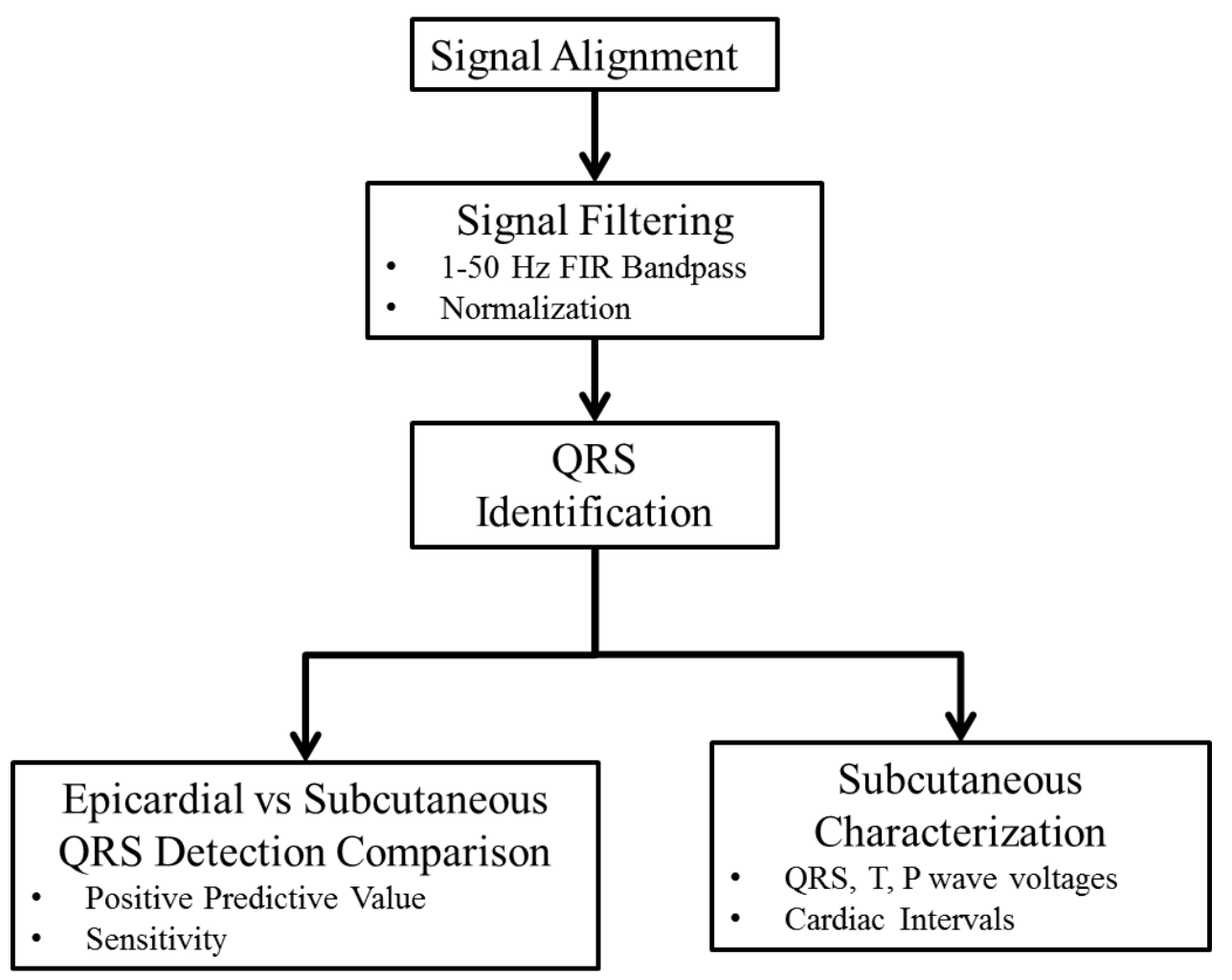

Figure 9. Summary of post-processing analysis of subcutaneous and epicardial ECG waveforms recorded in calves during normal activity and treadmill exercise. After the QRS identification, the $R-R$ interval was calculated, allowing for time-based isolation of the $P, Q R S$, and $T$ waves for further analysis.

\section{Signal Alignment}

First, the program aligns all three subcutaneous signals with the epicardial signal. This is essential because time delay between the driver output signal and the subcutaneous lead signals can cause a mismatch between the QRS peaks, hampering the detection algorithm.

The alignment subroutine first prompted the user to indicate the time frame of the first epicardial QRS wave. Because of the nature of the processed epicardial signal, the user is 
given several options to manipulate the signal for best QRS identification. These include inversion, second and third derivative functions. It then identifies the first QRS complex by performing a local maximum search for all three signals in the indicated time frame and aligns the signals based upon the matching of the peaks. The signals are then truncated to the same length in order to ease later processing and comparison. If, at any time, the program does not detect the epicardial signal or all three subcutaneous signals, it skips that data set and logs it as useless for QRS detection purposes.

\section{Signal Processing}

Initially, the author tried several approaches to filter the ECG signal for best QRS isolation. Combinations of a finite impulse response bandpass and Savitzky-Golay smoothing filter were attempted on raw data until the best combination with the smallest acceptable time delay was found in accordance with the average signal fast Fourier transform (FFT) (Figure 10).

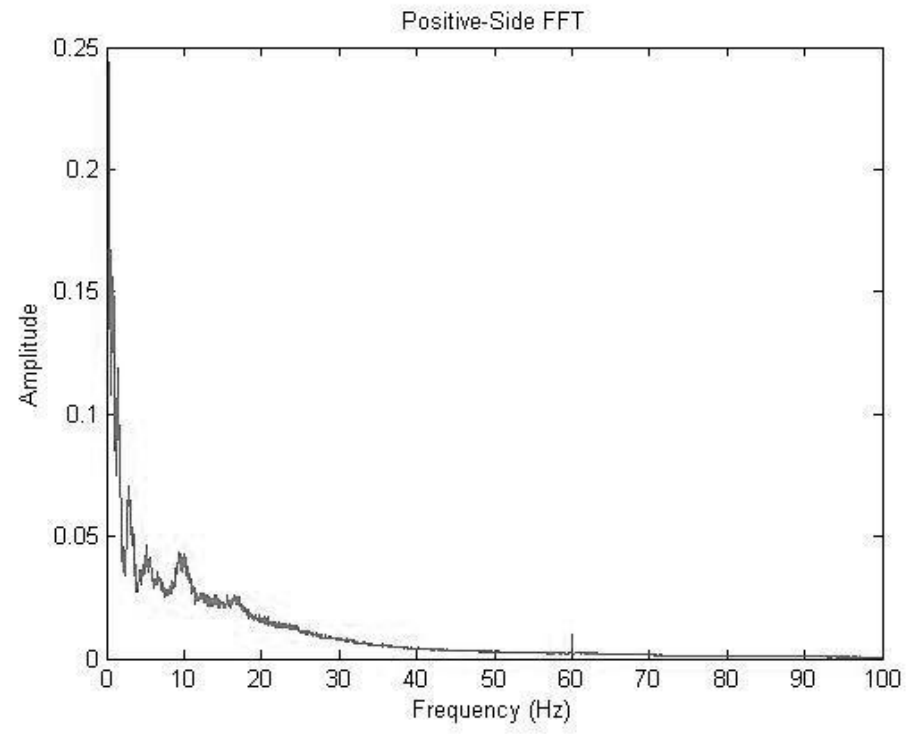

Figure 10. Average FFT of a subcutaneous lead pair over seven days. 
Ultimately, the Savitzky-Golay smoothing filter was unnecessary, and a simple 1-50 Hz (N=80) FIR bandpass filter was used to reject noise. Typical QRS detection filters often have bandpass filters with their upper limit in the range of $40-80 \mathrm{~Hz}$ for optimal detection $[44,45]$ with lower limits in the range of $0.5-1 \mathrm{~Hz}$ to reject low-frequency baseline drift [46]. The implemented filter only causes a time delay of $100 \mathrm{~ms}$, meaning that it could be theoretically realized in real-time driver software.

\section{QRS Detection}

The most intensive segment of the algorithm involved the coding of the QRS detection. The following is a summary of the implemented strategy which is a modified version of the SQRS method described by Pino et al [47]. First, the script asks for an initial threshold and blanking period as an input. It should be noted that the current Symphony driver requires a similar user input to initialize the QRS detection. The user inputs the best determined threshold for the first three QRS complexes along with an estimated blanking period in which, for the duration after successful QRS detection, the algorithm will ignore any subsequent incidents that reach the QRS threshold. After the program uses the bootstrap input to detect the first three complexes, it continues detecting new QRS complexes using $80 \%$ of the average of the past three thresholds and a blanking period of $80 \%$ of the average of the past three R-R intervals. If the next QRS amplitude or R-R interval varies from this average by more than $30 \%$, the algorithm ignores it in its average calculations. Detected QRS complexes are represented in an empty "detection matrix" with 1's. The script does this for all lead pairs and then compares their detection matrices against that of the epicardial signal to calculate true positives, false positives, 
and false negatives (Figure 11). Complexes that are detected within $170 \mathrm{~ms}$ of each other (less than twice the average length of a QRS complex) are considered "true positives"; all other detection combinations are categorized as either "false positives" (QRS detected in the subcutaneous signal but not in the epicardial signal) or "false negatives" (QRS detected in the epicardial signal but not in the subcutaneous signal). These were used to calculate the positive predictive value and sensitivity of each subcutaneous lead pair according to the following equations:

\section{Positive Predictive Value}

\section{Sensitivity}

\author{
True Positives \\ True Positives + False Positives
}

\section{True Positives}

True Positives + False Negatives

It is important to note that positive predictive value is an indication of the rate of a Type I error (false positive). While on counterpulsation therapy, a Type I error may result in an inappropriately timed device diastole, creating unstable and non-ideal hemodynamic conditions. Sensitivity indicates the rate of a Type II error (False Negative). A Type I error would simply result in a "skipped" device diastole, meaning that the patient has no counterpulsation support on that cardiac beat. Since the emerging devices are designed for partial circulatory support, the temporary absence of this support reduces the significance of this type of error when compared to the Type II error. 

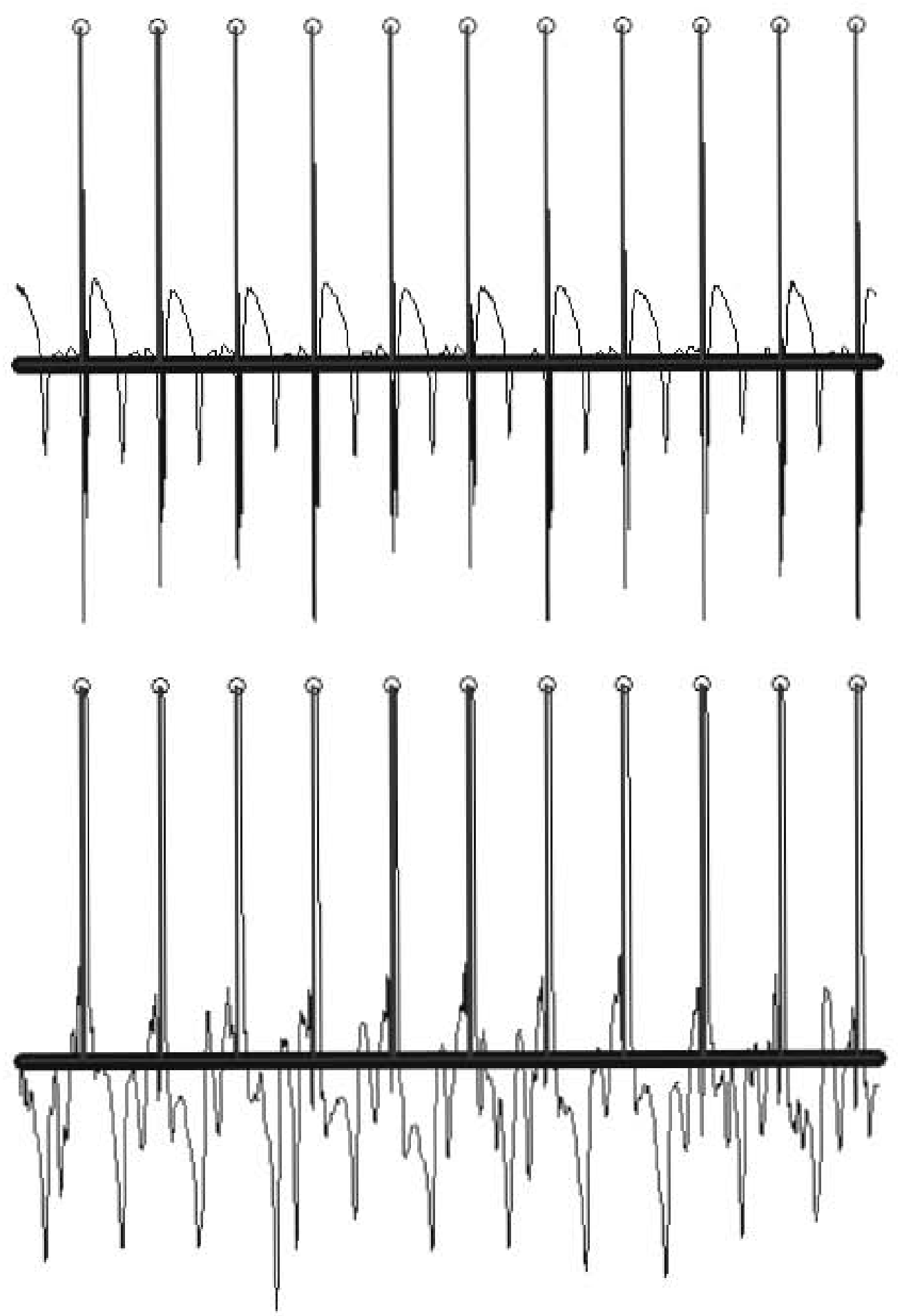

Figure 11. Sample ECG waveforms measured with epicardial (top) and subcutaneous (bottom) leads plotted over the stem plot of the detection matrix. The vertical thick line indicates a detected $Q R S$ complex. 


\section{Subcutaneous Characterization}

Once the program has detected a "normal" R-R interval, it uses the time capture method to isolate the P, QRS and T waves. Basically, it takes specified percentages of the R-R interval and labels them as each cardiac wave. Once it has done this, it calculates the voltage change for each cardiac wave and attempts to locate the peaks using a local maximum or minimum method. For the QRS complex, a "moving average" detection algorithm "rides" the peak up and down, marking the length of the peak in order to calculate QRS length. Once all of these values are accounted for, the major cardiac intervals are calculated using a peak-to-peak method. All statistics were performed in Minitab (Minitab Inc, State College, PA). 


\section{CHAPTER III - RESULTS}

Experimental Bovine Data

2,818 thirty-second data epochs were recorded at rest and 24 data epochs recorded during treadmill exercise (15-20 minutes each) for and aggregated total of 32 hours of recorded subcutaneous ECG data. Data sets were excluded when the ECG waveforms measured with the epicardial or any of the three subcutaneous leads were missing due to unintended disconnection from the data acquisition system. Sample ECG waveforms for epicardial and subcutaneous lead configurations during normal activity and treadmill exercise are shown in Figure 12.

\section{Epicardial and Subcutaneous QRS Triggering Comparison}

Using the described QRS detection algorithm with the epicardial signal as the control, the triggering rate for the subcutaneous ECG leads demonstrated $99.1 \pm 0.4 \%$ positive predictive value and $96.8 \pm 1.5 \%$ sensitivity during normal daily activity, and $98.0 \pm 10.2 \%$ positive predictive value and $93.3 \pm 5.6 \%$ sensitivity during treadmill exercise (Table 1 ). No set of lead pairs showed a statistically significant difference in positive predictive value or sensitivity compared to the other configurations $(\alpha=.05)$. Triggering rate was not significantly affected by QRS, T, or P voltage as determined by multivariate regression and best-subset analysis.

The mean QRS voltage was $818 \pm 99 \mu \mathrm{V}$, the mean T-wave voltage was $371 \pm 55 \mu \mathrm{V}$, and the mean-P wave voltage $256 \pm 48 \mu \mathrm{V}$. No abnormalities were observed in any of the standard cardiac intervals. 
At Rest

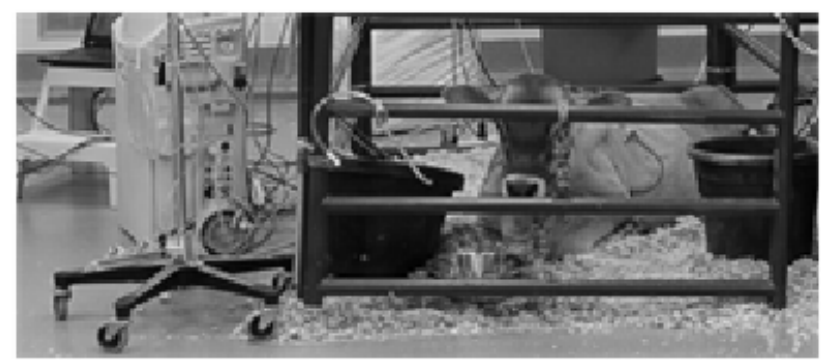

Processed Epicardial ECG

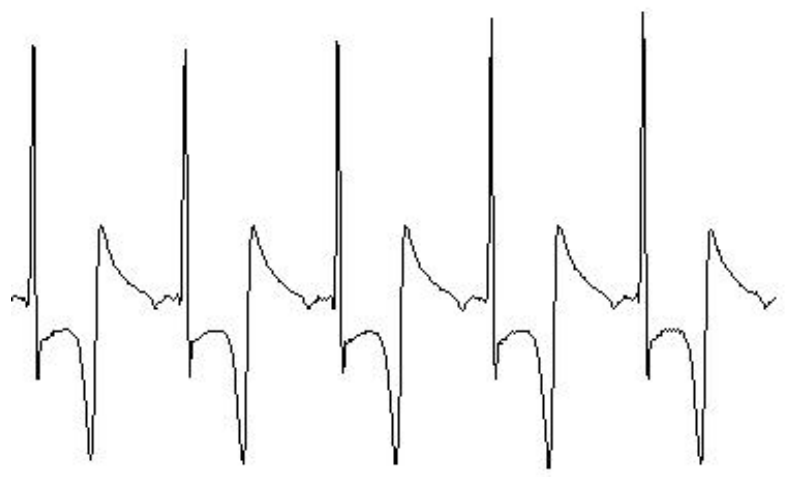

Subcutaneous ECG

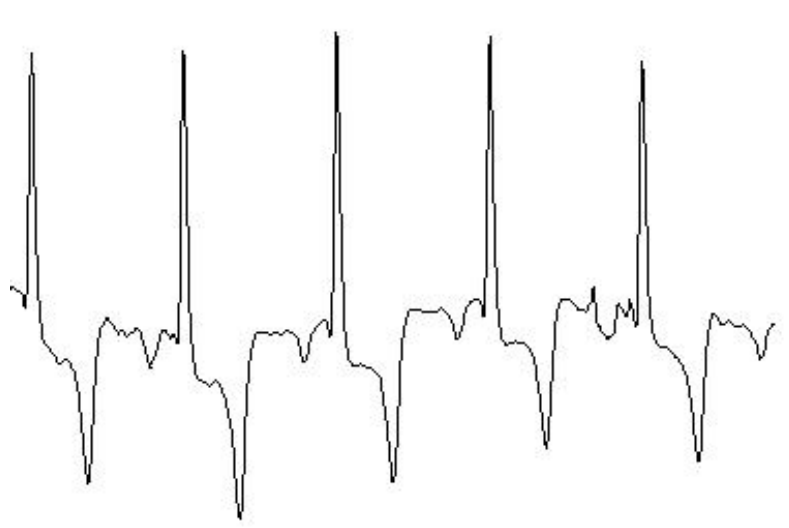

Treadmill Exercise

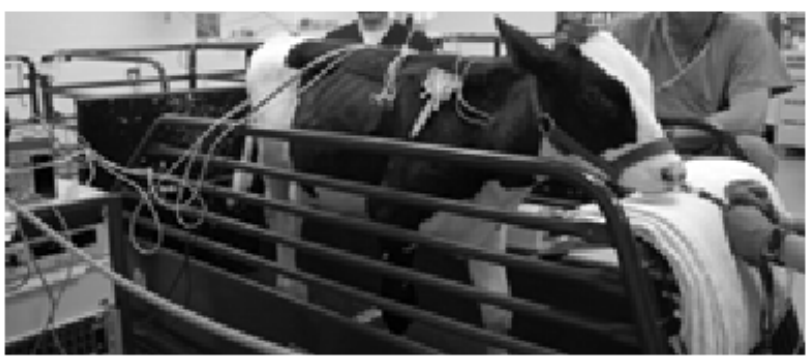

Processed Epicardial ECG

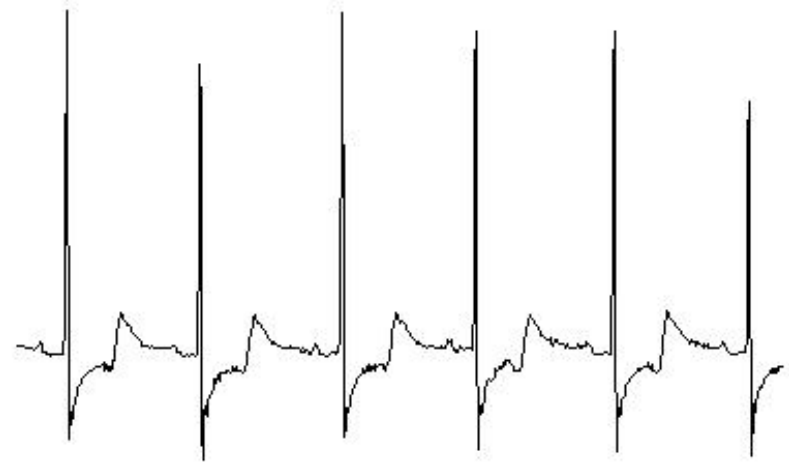

Subcutaneous ECG

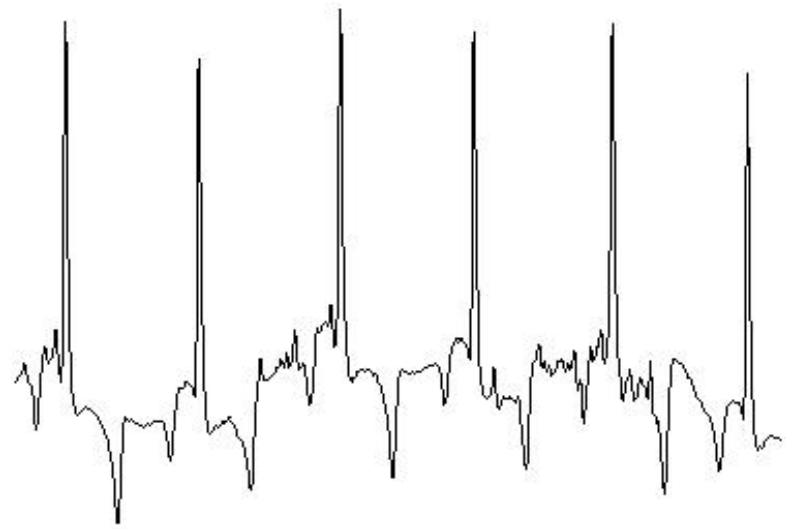

Figure 12. Subcutaneous versus epicardial ECG signals for both normal calf activity (left) and during treadmill exercise (right). 
At Rest Data (30 second clips, $400 \mathrm{~Hz}$ )

\begin{tabular}{|c|c|c|c|c|c|c|c|}
\hline $\mathbf{N}$ & SubQ Pair & Epochs & PPV & Sensitivity & R Wave (mV) & T Wave (mV) & P Wave (mV) \\
\hline \multirow{4}{*}{$\mathbf{1}$} & Pair 1 & 137 & $0.999 \pm .001$ & $0.996 \pm .002$ & 1.123 & 0.524 & 0.534 \\
\cline { 2 - 8 } & Pair 2 & 125 & $0.971 \pm .020$ & $0.925 \pm .021$ & 0.964 & 0.597 & 0.405 \\
\cline { 2 - 8 } & Pair 3 & 135 & $0.997 \pm .002$ & $0.972 \pm .006$ & 0.549 & 0.175 & 0.125 \\
\hline \multirow{4}{*}{2} & Pair 1 & 158 & $0.999 \pm .001$ & $0.998 \pm .001$ & 1.147 & 0.478 & 0.234 \\
\cline { 2 - 8 } & Pair 2 & 157 & $0.998 \pm .001$ & $0.994 \pm .003$ & 0.845 & 0.337 & 0.169 \\
\cline { 2 - 8 } & Pair 3 & 151 & $0.995 \pm .002$ & $0.987 \pm .006$ & 0.639 & 0.342 & 0.216 \\
\hline \multirow{4}{*}{3} & Pair 1 & 101 & $0.986 \pm .012$ & $0.962 \pm .013$ & 0.202 & 0.243 & 0.206 \\
\cline { 2 - 8 } & Pair 2 & 158 & $0.995 \pm .007$ & $0.984 \pm .008$ & 0.691 & 0.207 & 0.147 \\
\cline { 2 - 8 } & Pair 3 & 95 & $0.993 \pm .012$ & $0.992 \pm .012$ & 0.831 & 0.209 & 0.159 \\
\hline \multirow{4}{*}{4} & Pair 1 & 160 & $0.996 \pm .002$ & $0.992 \pm .003$ & 1.035 & 0.320 & 0.220 \\
\cline { 2 - 8 } & Pair 2 & 83 & $0.994 \pm .003$ & $0.932 \pm .009$ & 0.828 & 0.334 & 0.239 \\
\cline { 2 - 8 } & Pair 3 & 83 & $0.969 \pm .014$ & $0.890 \pm .033$ & 0.591 & 0.256 & 0.161 \\
\hline \multirow{4}{*}{5} & Pair 1 & 254 & $0.982 \pm .012$ & $0.969 \pm .011$ & 0.360 & 0.258 & 0.135 \\
\cline { 2 - 8 } & Pair 2 & 263 & $0.999 \pm .001$ & $0.997 \pm .001$ & 0.880 & 0.237 & 0.157 \\
\cline { 2 - 8 } & Pair 3 & 249 & $0.985 \pm .005$ & $0.968 \pm .013$ & 0.717 & 0.328 & 0.202 \\
\hline \multirow{4}{*}{$\mathbf{6}$} & Pair 1 & 201 & $0.996 \pm .004$ & $0.986 \pm .007$ & 0.944 & 0.475 & 0.374 \\
\cline { 2 - 8 } & Pair 2 & 153 & $0.991 \pm .004$ & $0.935 \pm .016$ & 0.953 & 0.458 & 0.341 \\
\cline { 2 - 8 } & Pair 3 & 155 & $0.990 \pm .005$ & $0.950 \pm .012$ & 0.866 & 0.418 & 0.284 \\
\hline
\end{tabular}

Treadmill Data (10-20 minute clips, $400 \mathrm{~Hz})$

\begin{tabular}{|c|c|c|c|c|c|c|c|}
\hline $\mathbf{N}$ & SubQ Pair & Epochs & PPV & Sensitivity & R Wave (mV) & T Wave (mV) & P Wave (mV) \\
\hline \multirow{4}{*}{5} & Pair 1 & 5 & $0.965 \pm .076$ & $0.972 \pm .035$ & 0.660 & 0.283 & 0.173 \\
\cline { 2 - 8 } & Pair 2 & 4 & $0.992 \pm .016$ & $0.985 \pm .021$ & 1.045 & 0.424 & 0.259 \\
\cline { 2 - 8 } & Pair 3 & 3 & $0.986 \pm .064$ & $0.922 \pm .110$ & 1.003 & 0.383 & 0.221 \\
\hline \multirow{4}{*}{$\mathbf{6}$} & Pair 1 & 6 & $0.974 \pm .012$ & $0.919 \pm .039$ & 0.923 & 0.664 & 0.486 \\
\cline { 2 - 8 } & Pair 2 & 3 & $0.969 \pm .067$ & $0.839 \pm .290$ & 0.778 & 0.452 & 0.278 \\
\cline { 2 - 8 } & Pair 3 & 3 & $0.992 \pm .023$ & $0.960 \pm .067$ & 1.060 & 0.492 & 0.418 \\
\hline
\end{tabular}

Note: When applicable, $95 \%$ confidence interval upper bound does not exceed 1 .

Table 1. Positive Predictive Value (PPV), Sensitivity, and ECG landmark voltages for all ECG lead pairs at rest and during treadmill exercise. 


\section{Lead Implantation and Migration}

The surgical technique for implanting the subcutaneous ECG leads was developed using human cadavers and during acute animal experiments, as previously described. Subcutaneous leads were accurately delivered at distances up to $15 \mathrm{~cm}$ from the incision using the straight-needle method. The average time for placement of a single lead was 35 \pm 6 seconds. For placement of a full set of six subcutaneous leads, the average total time was $9 \pm 2$ minutes. All leads (36/36) were within $1.4 \mathrm{~cm}$ of their initial placement with an average migration of $0.52 \pm 0.10 \mathrm{~cm}$ (Figure 13). Surgical exposure at necropsy confirmed that none of the subcutaneous leads had significant migration within the subcutaneous space. Electrode position did not significantly increase lead migration, and average lead migration did not vary between animals $(\alpha=0.05)$.
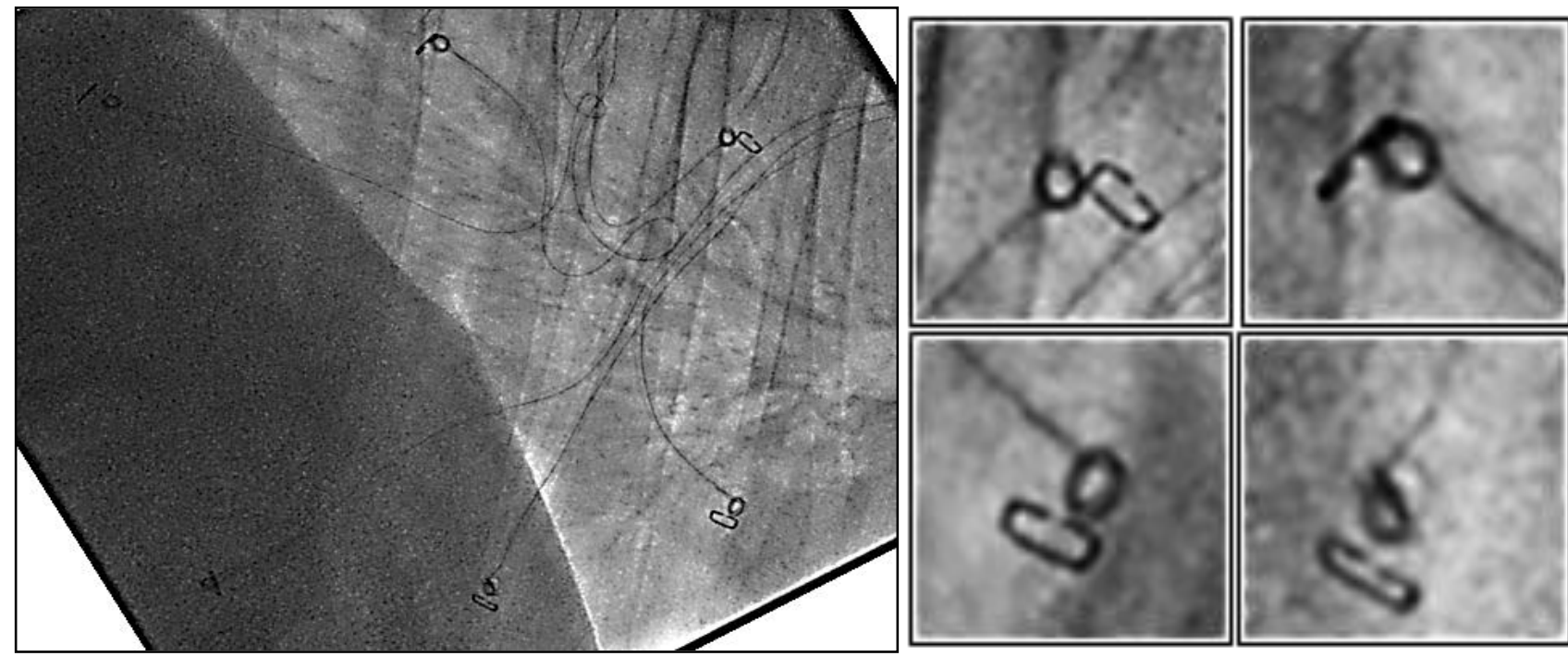

Figure 13. Fluoroscopic digital image of implanted ECG subcutaneous leads at 14-days post-implant. Staples were placed on the skin at time of implant to mark placement of the subcutaneous leads (left). Image reveals no significant migration of the subcutaneous leads (right), which was quantified using ImageJ (NIH, Bethesda MD) analysis software. 


\section{Lead Durability}

There were no lead fractures, sensing wire damage, or permanent signal loss for all tested leads $(36 / 36,100 \%)$. In all calves, no sign of lead infection was observed or identified at necropsy. Temporary loss of signal occasionally resulted from the disconnection of the external pin and socket connectors. This was intended, by design, to prevent the animals from accidentally pulling out the subcutaneous leads. 


\section{CHAPTER IV - DISCUSSION, LIMITATIONS AND CONCLUSION}

\section{Discussion}

Current counterpulsation therapies utilize surface ECGs for triggering of device systole and diastole. However, with the emergence of chronic counterpulsation devices, a more robust, long-term ECG strategy is necessary. It is essential that these devices are triggered accurately and precisely in order to prevent unstable hemodynamic conditions.

While studies have examined the R-wave detection performance between subcutaneous and surface ECGs in an implantable loop recorder [39], surface leads are not optimal for long-term triggering of a chronic counterpulsation device. Epicardial and endocardial leads offer quality signals for QRS detection, but unwantedly increase operative complexity and postoperative complications. In order for subcutaneous leads to act as adequate surrogates for cardiac leads, they must be easily implantable, demonstrate no long-term migration, and offer robust ECG signals for QRS detection. The fabricated subcutaneous leads were easily implanted using a minimally invasive technique and standard surgical equipment. Fluoroscopy confirmed that the leads did not migrate over time, demonstrating potential for use with a chronic device.

Uniquely positioned in the subcutaneous space, treadmill exercise of the monitored animals introduces significant motion and muscular (electric) artifacts into the subcutaneous signals. These artifacts did not significantly impair QRS detection and were 
minimized with signal processing. QRS detection analysis demonstrated that exercise did not significantly impair Symphony triggering rates; positive predictive values remained above $95 \%$ and sensitivity values remained above $84 \%$ (mean $93.3 \pm 5.6 \%$ ). The location of the subcutaneous leads provided excellent $\mathrm{R}$ wave and moderate $\mathrm{T}$ wave amplitudes ensuring that "oversensing" was not a problem.

\section{Limitations}

This feasibility study has several limitations. Due to the nature of the concomitant circulatory support device implantation, the epicardial signal was first sent through the circulatory support device driver to serve as the trigger for the device ejection. An output from this was fed into the data acquisition system and recorded by LabVIEW. Epicardial signal was heavily processed by the driver algorithm and the original signal could not be recovered. This meant that important ECG landmark and statistical comparisons (such as root mean square noise and signal subtraction) could not be made. Regardless, the QRS triggering analysis remains the most essential element of the comparison. Due to the volume of data, epicardial leads were used as the control and QRS complexes were not visually identified for each epoch. Any false positives or false negatives in the epicardial signal cannot be accounted for. While the fabricated leads were sufficient for use in large quadrupeds, they remain inappropriate for human implantation without further development and evaluation. The chronic study end point of 14 days remains insufficient to quantify long-term lead migration (6 months -2 years), durability and histological response. 
The QRS detection algorithm has several limitations. First, the algorithm utilizes an adaptive thresholding technique for identifying QRS complexes. It should be noted that while this is an accepted algorithm and still often used in the industry, it is a fairly dated strategy and does not take into account advances in ECG wavelet analysis and other emerging techniques. However, these new strategies can be implemented in future versions of the script.

Another limitation is that the ECG landmark analysis is not comprehensive; the script performs "peak-to-peak" interval analysis which does not give, for example, the exact PR interval or PR segment (Note: The PR interval is the distance from the start of the P wave to the beginning of the QRS complex. The PR Segment is the distance between the end of the $\mathrm{P}$ wave and the beginning of the QRS segment). Instead, it gives the distance from the peak of the QRS complex to the peak of the $\mathrm{P}$ wave - a useful number, but not a clinical standard. Improvements in $\mathrm{P}$ and $\mathrm{T}$ wave isolation through peak and valley detection methods are possible solutions to this problem.

Finally, the bootstrapping of the program for each ECG epoch means that it takes a significant amount of time to analyze a large number of signals. While the program does record the bootstrap of every epoch it has analyzed in case a segment needs to be reviewed, it still needs an initial user input. This can be remedied by a system that uses the average bootstrapping threshold and blanking period to initialize the next epoch. This would have to be carefully implemented to reduce "bad" bootstrapping and subsequent poor QRS detection. 


\section{Conclusion}

The study objective to develop subcutaneous ECG leads for use with Symphony device and wearable pneumatic drier was achieved as evidenced by successful implantation of leads and completion of chronic bovine experiments. The hypothesis that subcutaneous leads provide equivalent QRS detection, lead migration, and durability as clinicalstandard epicardial leads was also proven as evidenced by (1) equivalent QRS detection rates at rest ( $>95 \%$ positive predictive value and sensitivity) and exercise ( $>90 \%$ positive predictive value and sensitivity), (2) lead migration of less than $1.4 \mathrm{~cm}$ in all leads, and (3) no lead fracture, permanent signal loss, or infection. These findings demonstrate the feasibility of subcutaneous leads for synchronized timing of mechanical circulatory support while offering the advantages of less invasive surgery and associated risk factors. 


\section{CHAPTER V - Works Cited}

1. Koenig, S.C., et al., Development and early testing of a simple subcutaneous counterpulsation device. ASAIO J, 2006. 52(4): p. 362-7.

2. Giridharan, G.A., et al., Predicted hemodynamic benefits of counterpulsation therapy using a superficial surgical approach. ASAIO J, 2006. 52(1): p. 39-46.

3. Lloyd-Jones, D., et al., Heart disease and stroke statistics-2010 update. Circulation, 2010. 121(7): p. e46-e215.

4. Rosamond, W., et al., Heart disease and stroke statistics--2008 update: a report from the American Heart Association Statistics Committee and Stroke Statistics Subcommittee. Circulation, 2008. 117(4): p. e25.

5. Muntwyler, J., et al., One-year mortality among unselected outpatients with heart failure. Eur Heart J, 2002. 23(23): p. 1861-6.

6. Pitt, B., et al., The effect of spironolactone on morbidity and mortality in patients with severe heart failure. Randomized Aldactone Evaluation Study Investigators. N Engl J Med, 1999. 341(10): p. 709-17.

7. e-Statistics Report on Transplant, Waiting List and Donor Statistics, in 2008 Summary Statistics, January 1 to December 31st, 2008. 2008, Canadian Institute for Health Information.

8. Koenig, S.C., et al., Acute hemodynamic efficacy of a 32-ml subcutaneous counterpulsation device in a calf model of diminished cardiac function. ASAIO journal (American Society for Artificial Internal Organs: 1992), 2008. 54(6): p. 578.

9. Moskowitz, A.J., E.A. Rose, and A.C. Gelijns, The cost of long-term LVAD implantation. The Annals of thoracic surgery, 2001. 71(3): p. S195-S198.

10. Westaby, S. and P. Poole-Wilson, Mechanical circulatory support in the UK. BMJ: British Medical Journal, 2007. 334(7586): p. 167.

11. Reinlib, L. and W. Abraham, Recovery from heart failure with circulatory assist: a working group of the National, Heart, Lung, and Blood Institute. J Card Fail, 2003. 9(6): p. 459-63.

12. Nanas, J.N. and S.D. Moulopoulos, Counterpulsation: historical background, technical improvements, hemodynamic and metabolic effects. Cardiology, 1994. 84(3): p. 156-67.

13. Scheidt, S., et al., Intra-aortic balloon counterpulsation in cardiogenic shock. Report of a co-operative clinical trial. N Engl J Med, 1973. 288(19): p. 979-84.

14. Lefemine, A.A., et al., Assisted circulation. III. The effect of synchronized arterial counterpulsation on myocardial oxygen consumption and coronary flow. Am Heart J, 1962. 64: p. 789-95.

15. Papaioannou, T.G. and C. Stefanadis, Basic principles of the intraaortic balloon pump and mechanisms affecting its performance. ASAIO J, 2005. 51(3): p. 296300.

16. Bolooki, H., ed. Clinical Application of the Intra-Aortic Baloon Pump. 3rd ed. 1998, Futura: New York. 
17. Santa-Cruz, R.A., M.G. Cohen, and E.M. Ohman, Aortic counterpulsation: a review of the hemodynamic effects and indications for use. Catheter Cardiovasc Interv, 2006. 67(1): p. 68-77.

18. Tsagalou, E.P., M.I. Anastasiou-Nana, and J.N. Nanas, Intra-aortic balloon counterpulsation for the treatment of myocardial infarction complicated by acute severe heart failure. Congest Heart Fail, 2009. 15(1): p. 35-40.

19. Dunkman, W.B., et al., Clinical and hemodynamic results of intraaortic balloon pumping and surgery for cardiogenic shock. Circulation, 1972. 46(3): p. 465-77.

20. Weiss, A.T., et al., Regional and global left ventricular function during intraaortic balloon counterpulsation in patients with acute myocardial infarction shock. Am Heart J, 1984. 108(2): p. 249-54.

21. Kern, M.J., et al., Enhanced coronary blood flow velocity during intraaortic balloon counterpulsation in critically ill patients. J Am Coll Cardiol, 1993. 21(2): p. 359-68.

22. Ishihara, M., et al., Effects of intraaortic balloon pumping on coronary hemodynamics after coronary angioplasty in patients with acute myocardial infarction. Am Heart J, 1992. 124(5): p. 1133-8.

23. Bhayana, J.N., et al., Effects of intraaortic balloon pumping on organ perfusion in cardiogenic shock. J Surg Res, 1979. 26(2): p. 108-13.

24. Sugita, Y., et al., The effect of intraaortic balloon pumping (IABP) on pulmonary circulation. Trans Am Soc Artif Intern Organs, 1985. 31: p. 389-94.

25. Hilberman, M., et al., Effect of the intra-aortic balloon pump upon postoperative renal function in man. Crit Care Med, 1981. 9(2): p. 85-9.

26. Jeevanandam, V., et al., Circulatory assistance with a permanent implantable IABP: initial human experience. Circulation, 2002. 106(12 Suppl 1): p. I183-8.

27. Sun, B. Sunshine: Chronic Aortic Counterpulsation for Class III Heart Failure. in Transcatheter Cardiovascular Therapeutics. 2009. San Francisco, CA.

28. Koenig, S.C., et al., Development and early testing of a simple subcutaneous counterpulsation device. ASAIO journal (American Society for Artificial Internal Organs: 1992), 2006. 52(4): p. 362.

29. Goldich, G., Getting in sync with intra-aortic balloon pump therapy. Nursing2011 Critical Care, 2011. 6(3): p. 14.

30. Lobodzinski, S.S. and M.M. Laks, Comfortable textile-based electrocardiogram systems for very long-term monitoring. Cardiology journal, 2008. 15(5): p. 477.

31. Kautzner, J., et al., Technical aspects of implantation of LV lead for cardiac resynchronization therapy in chronic heart failure. Pacing and clinical electrophysiology, 2004. 27(6p1): p. 783-790.

32. Schwartzman, D., et al., Postoperative lead-related complications in patients with nonthoracotomy defibrillation lead systems. Journal of the American College of Cardiology, 1995. 26(3): p. 776-786.

33. Lawton, J.S., et al., Sensing lead-related complications in patients with transvenous implantable cardioverter-defibrillators. The American Journal of Cardiology, 1996. 78(6): p. 647-651.

34. Bardy, G.H., et al., An entirely subcutaneous implantable cardioverterdefibrillator. N Engl J Med, 2010. 363(1): p. 36-44. 
35. Grace, A., et al., Evaluation of four distinct subcutaneous implantable

defibrillator (S-ICD®) lead systems in humans. Heart Rhythm, 2006. 3: p. S128-

S129.

36. Iglesias, J.F., et al., The implantable loop recorder: a critical review.

Kardiovaskuläre Medizin, 2009. 12(3): p. 85-93.

37. Ho, C. and S. Kurtzman, Three perspectives of cardiac electrical activity.

Biomedical sciences instrumentation, 2001. 37: p. 325.

38. Fotuhi, P., et al., $R$ Wave Detection by Subcutaneous ECG. Possible Use for Analyzing $R R$ Variability. Annals of noninvasive electrocardiology, 2001. 6(1): p. 18-23.

39. Bellardine, B., et al., Is Surface ECG a Useful Surrogate for Subcutaneous ECG? Pacing and Clinical Electrophysiology, 2010. 33(2): p. 135-145.

40. van Dam, P., et al., Improving sensing and detection performance in subcutaneous monitors. Journal of Electrocardiology, 2009. 42(6): p. 580-583.

41. Hassler, C., ECG lead placement in quadrupeds. Telemetry Times (Technical Note, 8/3/94). Data Sciences Int, 1994.

42. Koenig, S.C., et al., Integrated data acquisition system for medical device testing and physiology research in compliance with good laboratory practices.

Biomedical Instrumentation \& Technology, 2004. 38(3): p. 229-240.

43. Drew, G. and S. Koenig, Biomedical patient monitoring, data acquisition, and playback with LabVIEW. Olansen JB, Rosow E. Virtual Bio-Instrumentation: Biomedical, Clinical, and Healthcare Applications in LabVIEW, Prentice Hall, Upper Saddle River, NJ, 2002: p. 180-186.

44. Gomes, J.A., et al., Optimal bandpass filters for time-domain analysis of the signal-averaged electrocardiogram. The American journal of cardiology, 1987. 60(16): p. 1290-1298.

45. Thakor, N.V., J.G. Webster, and W.J. Tompkins, Estimation of QRS complex power spectra for design of a QRS filter. Biomedical Engineering, IEEE Transactions on, 1984(11): p. 702-706.

46. Frankel, R.A., et al., A filter to suppress ECG baseline wander and preserve STsegment accuracy in a real-time environment. Journal of electrocardiology, 1991. 24(4): p. 315-323.

47. Pino, E., et al. Real-Time ECG Algorithms for Ambulatory Patient Monitoring. 2005: American Medical Informatics Association. 


\section{EDUCATION}

M. Eng. in Bioengineering

B. S. in Bioengineering
Expected July 2011

Received May 2010

J.B. Speed School of Engineering, UofL, Louisville, KY

GPA $3.98 / 4.0$

Dates attended: 8/2006 - present Hours Completed: 133 Graduated Summa Cum Laude

High School Diploma

North Oldham High School, Goshen, KY

May 2006

Graduated Summa Cum Laude

\section{ACADEMIC AWARDS AND HONORS}

1. Rolando "Chip" Cheng, Jr. Memorial Scholarship - 2010

Recipient

Recognizes a bioengineering student in the graduating class whose character reflects humanitarianism, innovative mind, and entrepreneurial spirit.

2. Jerry and Pat Sturgeon Academic Excellence Award - 2010 Recipient Awarded annually to the student achieving the highest scholastic performance after completing their Bachelor of Science degree in Bioengineering.

3. U of L Trustee's Scholarship

Full Tuition, room and board for five years.

4. Dean's List - Fall 2006, Spring 2009, Fall 2009

5. Dean's Scholar - Spring 2007, Summer 2007, Fall 2007, Spring 2008, Summer 2008,

6. Tau Beta Pi Engineering Honor Society

7. $2^{\text {nd }}$ Place Undergraduate Poster, Development of Microscale Polymer Fibers as Scaffolds for the Engineering of Tissue, University of Louisville Engineering Exposition, 2008 


\section{EMPLOYMENT HISTORY}

Spence Cardiac Research Inc, Louisville, KY Co-op Engineer under Paul Spence, MD

May 2009 - Oct 2010

- Work with an international team of engineers and physicians to develop and test medical products

- Design, prototype and test early-stage medical devices in the fields of atrial fibrillation and sports medicine (preliminary patents filed)

- Design and test components, and assist in preGLP animal and cadaver studies for a counterpulsation cardiac assist device

- Assist in writing and editing NIH and statefunded grant applications for the development of medical products

Ethicon, Somerville, NJ Co-op in R\&D Ethicon Products

Aug 2008 - Dec 2008

- Designed, fabricated and tested prototype devices for next-generation wound closure - Drafted design plan for discovery-phase project

- Participated in a cadaver lab and received OR observation training

University of Louisville, Louisville, $\mathrm{KY}$ Member of the Bio-MEMS Research Team June 2007 - May 2009

- Collaborated with a team of engineers and professors on university PhD-level and Masterlevel research projects

- Performed sterile cell culture, fluorescent staining, and ELISA assay analysis

- Operated and programmed micromill machine for direct-write fabrication of polymer nanofibers

Sonopress USA, Louisville, KY

Intern under Eric Jones, M. Eng. June 2006 - Aug 2006

- Monitored line productivity

- Created production efficiency charts

- Worked with superiors to increase line efficiency 
STATE AND FEDERAL EXTRAMURAL FUNDING

NIH SBIR PHASE II GRANT: 2R44HL083586-02A1

Development of a Counterpulsation Therapy Device

August 2008 - July 2010

Award Amount: \$1,437,630

Role: Biomedical Engineer

Assist in device animal studies and the collection and analysis of necropsy data.

NIH SBIR PHASE II GRANT: 2R44HL088760-02

Portable Pneumatic Driver for Counterpulsation Therapy

May 2009 - May 2011

Award Amount: \$1,580,460

Role: Biomedical Engineer

Assist in driver animal studies and the collection and analysis of necropsy data.

NIH SBIR PHASE I GRANT: 1R43HL102981-01

Counterpulsation Device with Integrated ECG Sensing

May 2010 - October 2010

Award Amount: \$261,965

Role: Biomedical Engineer

Provide ECG design and fabrication support, participate in animal studies, and collect and analyze signaling data.

KENTUCKY SCIENCE AND TECHNOLOGY CORPORATION SBIR PHASE II MATCHING FUND:

KSTC-184-512-08-054

Development of a Counterpulsation Therapy Device

October 2008 - April 2010

Award Amount: $\$ 500,000$

Role: Co-op Student

Responsible for design, fabrication, and benchtop testing of prototype medical devices as well as participating in mock flow loop and animal model experiments by assisting with data collection and analysis.

KENTUCKY SCIENCE AND TECHNOLOGY CORPORATION SBIR PHASE II MATCHING FUND:

KSTC-184-512-10-077

Patient Friendly Driver for Counterpulsation Therapy

January 2010 - July 2011

Award Amount: \$430,000 
Role: Engineer

Complete the development of the portable driver in collaboration with LB Technology and complete testing and analysis of the display and patient messaging over a range of clinically relevant scenarios.

\section{PUBLISHED IN PEER-REVIEWED JOURNALS}

1. Ising M, Warren SP, Sobieski MA, Slaughter MS, Koenig SC, Giridharan GA. Flow Modulation Algorithms for Continuous Flow Left Ventricular Assist Devices to Increase Vascular Pulsatility: A Computer Simulation Study. Cardiovascular Engineering and Technology, Vol. 2, No. 2, June 2011.

2. Berry SM, Warren SP, Hilgart DA, Schworer AT, Gobin AS, Cohn RW, Keynton RS. Endothelial Cell Scaffolds Generated by $3 D$ Direct Writing of Biodegradable Polymer Microfibers. Biomaterials, Vol. 32, No. 7, March 2011.

\section{PUBLISHED IN PEER-REVIEWED CONFERENCE PROCEEDINGS}

1. Warren SP, Dowling R, Tompkins LH, Spence PA, Koenig SC, Giridharan GA, Sobieski MA, Sherwood L, Slaughter MS. Continued Development of a Long-Term Counterpulsation Device (Symphony) for Partial Circulatory Support. $21^{\text {st }}$ Century Treatment of Heart Failure: Synchronizing Surgical and Medical Therapies for Better Outcomes, Cleveland, $\mathrm{OH}$, 2010 .

2. Ising MS, Warren SP, Slaughter MS, Koenig SC, Sobieski MA, Giridharan GA. Delivering Physiologic Pulse Pressures with Continuous Flow Ventricular Assist Devices. $56^{\text {th }}$ Annual ASAIO Conference, Baltimore, MD, 2010.

3. Berry SM, Hilgart DA, Warren SP, Schworer AT, Gobin AS, Cohn RW, Keynton RS. Fabrication of Microvascular-Scale Polymer-Fiber Scaffolds for the Growth of Endothelial Cells. $5^{\text {th }}$ International Conference on Microtechnologies in Medicine and Biology, Quebec City, Quebec, 2009.

4. Berry SM, Cambron SD, Warren SP, Pabba S, Cohn RW, Keynton RS. Direct Write Fabrication of Polymer Fibers for 
Microscale Applications. $17^{\text {th }}$ Biennial UGIM, Louisville, KY, 2008.

\section{PATENT AND INTELLECTUAL PROPERTY}

1. System and Method for Providing Cardiac Support Ising MS, Warren SP, Sobieski MA, Slaughter MS, Koenig SC, Giridharan GA University of Louisville Research Foundation Provisional Patent Application Submitted, 2010

2. Ablation Catheter Device and Methods Spence PA, Warren SP, et al. SCR Inc. Pending Application Submission 2011

\section{LECTURES}

1. Subcutaneous ECG Cooperative Education Project Overview of work done at SCR Inc. on subcutaneous ECG animal trials.

Presented to the Speed School Industrial Board of Advisors University of Louisville, November 12, 2010

\section{Pediatric Cardiology}

Overview of pediatric hemodynamics, cardiovascular disorders, medical therapies and surgical procedures.

Presented to graduate-level class, Cardiovascular Dynamics I University of Louisville, July 12, 2010

\section{Embryonic Cardiogenesis}

Overview of embryonic cardiovascular development, fetal vs postnatal circulation, common cardiac congenital defects and therapies, and fetal cardiac surgery.

Presented to graduate-level class, Cardiovascular Dynamics I University of Louisville, June 21, 2010

4. Ventricular Assist Devices

Brief introduction to the technologies and applications of ventricular assist devices.

Presented to participants in the INSPIRE Program, a summer program targeting minority high school students with interests in engineering 
University of Louisville, June 9, 2010.

\section{CONFERENCES ATTENDED}

1. $\quad 21^{\text {st }}$ Century Treatment of Heart Failure -2010

2. American Society for Artificial Internal Organs - 2010

3. Kentucky Innovation and Entrepreneurship

Conference - 2010

4. Boston Atrial Fibrillation Symposium - 2010

5. International Conference on Microtechnologies in Medicine and Biology - 2009

6. University Government Industry Micro/Nano Symposium -2008

\section{SOCIETIES}

1. American Society for Artificial Internal Organs

2. Biomedical Engineering Society

3. American Association for the Advancement of Science

4. Tau Beta Phi Engineering Honor Society

5. Louisville Astronomical Society

6. Kappa Sigma Fraternity

\section{EXTRACURRICULAR ACTIVITIES}

1. University of Louisville Biomedical Engineering Society

- Vice President (2009-2010)

2. Kappa Sigma Fraternity

- Greek Week Chair

3. SSI Advanced Scuba Diver

- Stress and Rescue Certified

- Over 35 logged dives

4. Louisville Astronomy Society

\section{COMMUNITY SERVICE}

1. "Opposition Force" Volunteer, Fort Knox

Zussman Military

Training Center

2. St. James Art Festival Volunteer

3. U of L Spring Football Game Volunteer

4. Arcadia Community Center Childcare Volunteer

5. Louisville Astronomy Society Public Viewing 
CHAPTER VII - APPENDIX

I. EXTENDED STATISTICS TABLE

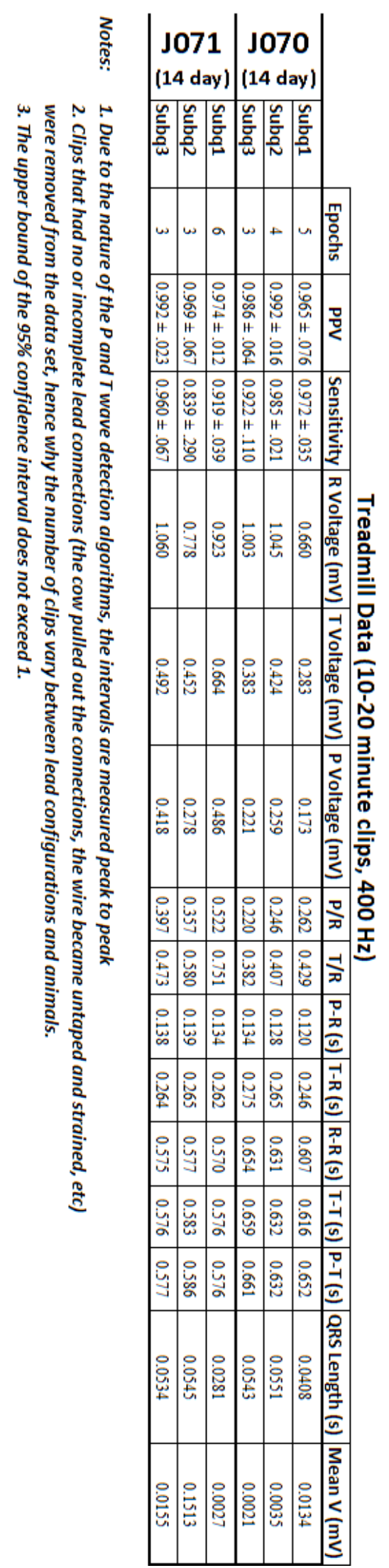

\begin{tabular}{|c|c|c|c|c|c|} 
J071 & J070 & J069 & J068 & J067 & J066 \\
(14 day) & (14 day) & (7 dav) & (7 dav) & (7 dav) & (7 dav) \\
\hline
\end{tabular}

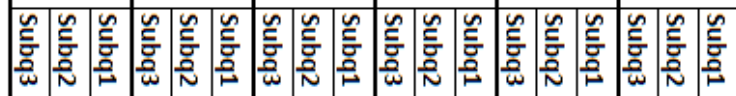

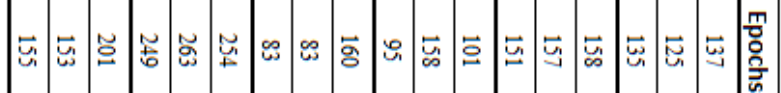

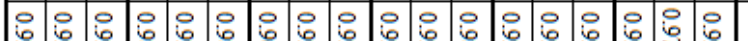

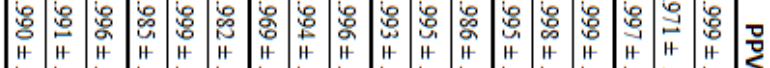

훙

o o o o o o o o o o b o o b o n

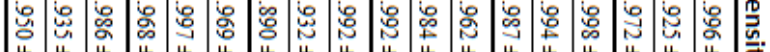

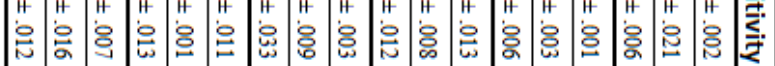

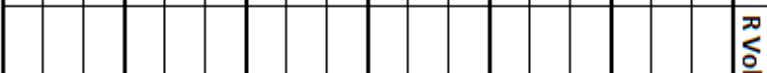

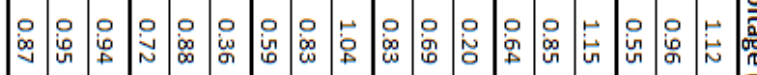

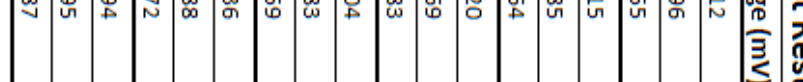

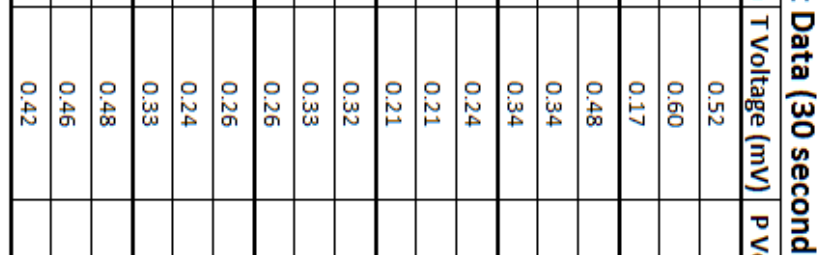

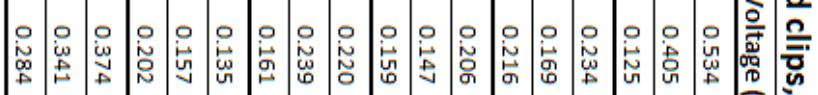

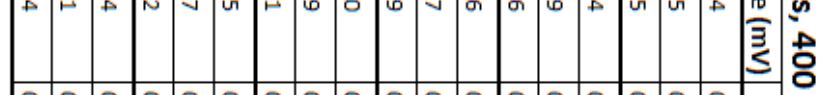

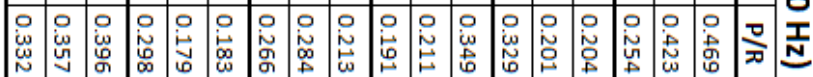

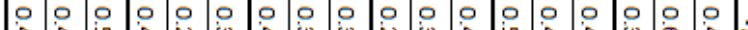

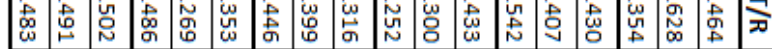

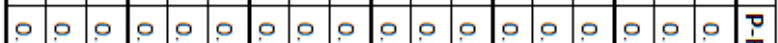

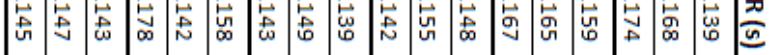
\begin{tabular}{llllllllllllllllllll}
\hline & 0 & 0 & 0 & 0 & 0 & 0 & 0 & 0 & 0 & 0 & 0 & 0 & 0 & 0 & 0 & 0 & 0 & -1 \\
\hline
\end{tabular}

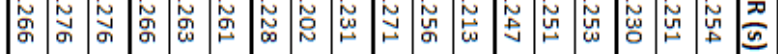

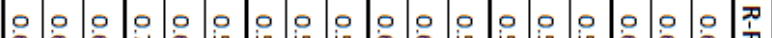

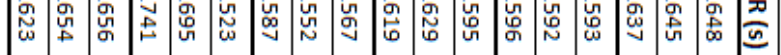

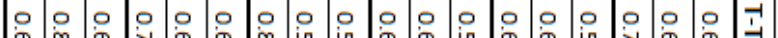

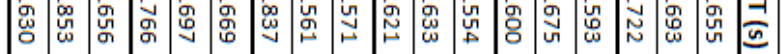

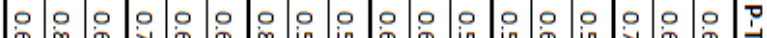

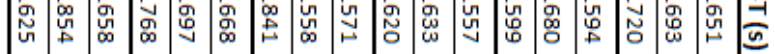

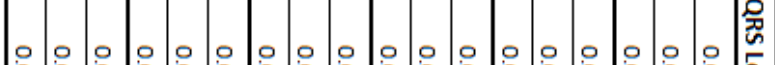

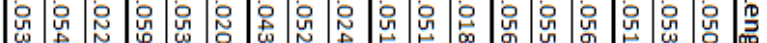

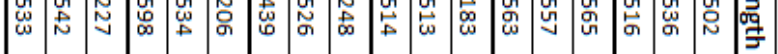

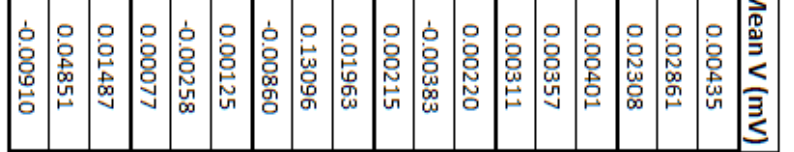




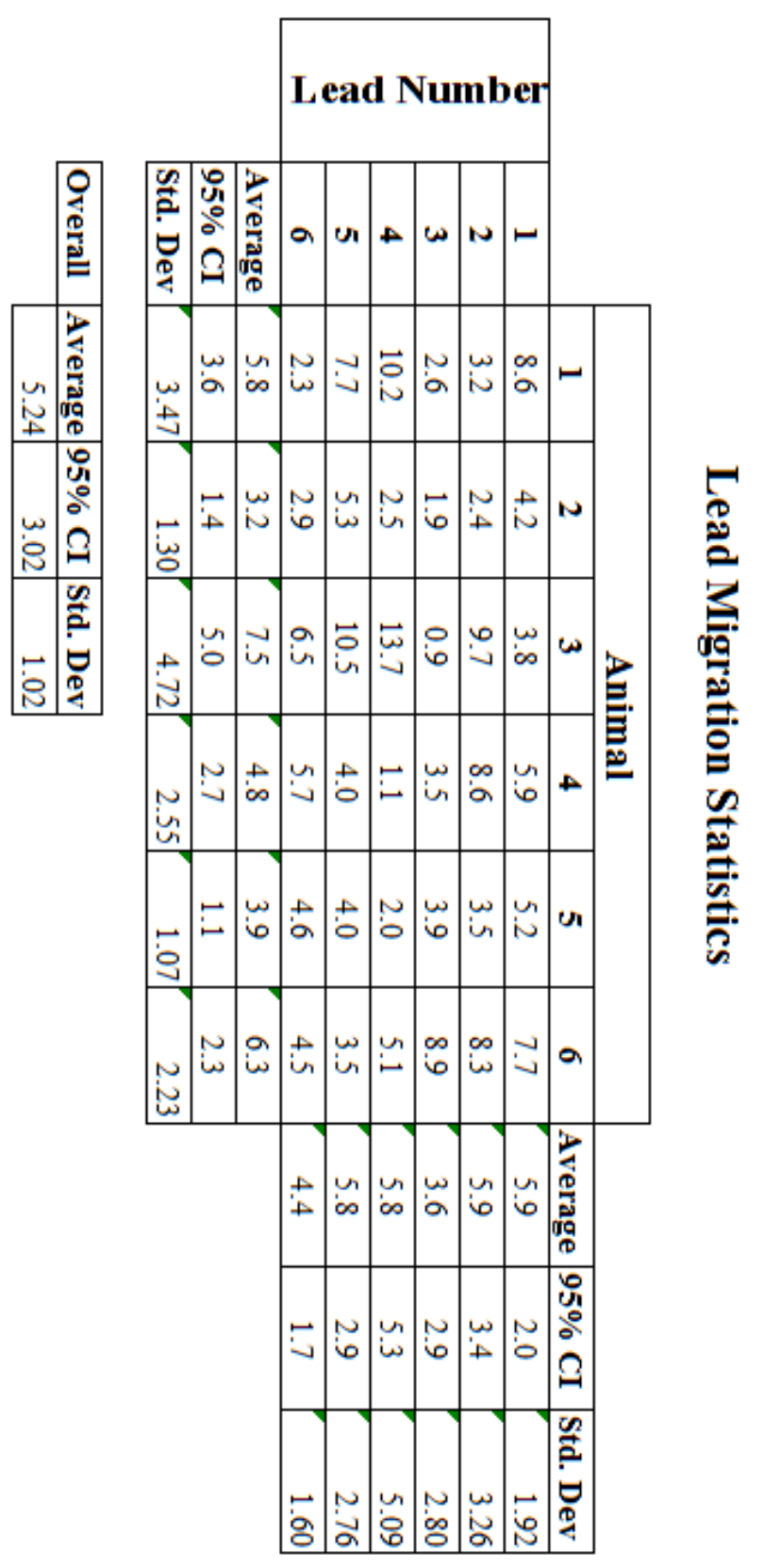




\section{CODE APPENDIX (Printed in columns)}

\section{A. Master Algorithm Code}

\% Test version of filtering for ECG signal \% By Sean Warren

\%Last modified: 6/6/2011

$\%$ Make sure you have an empty stats matrix (totalfiles x 65) of zeros

\%USE THESE COMMANDS BEFORE

STARTING A FRESH SET (without the \%) $\%$ stats=zeros(TOTALFILES,65);

\%save('stats.mat','stats');

clc

clear all

diary('J066_log.txt')

ampfactor $=10$;

counter $=273$;

fileend $=317$;

cow='J071';

$\mathrm{hz}=400$;

load('signalcheck.mat');

load('stats.mat');

for $\mathrm{i}=$ counter+1:1:fileend +1

save('stats.mat','stats')

hold off

filename $=\operatorname{sprintf(} \% \mathrm{~s} \% \mathrm{~s} \% \mathrm{i} \% \mathrm{~s}^{\prime}, \mathrm{cow},{ }_{-}$, ,i-

1,'.mat'); \% Treadmill_for treadmill

$\operatorname{disp}\left(\operatorname{sprintf}\left(' \% s^{\prime,}, '\right)\right)$

disp(sprintf('\%s','-

disp(sprint('\%s\%s', 'File ', filename))

disp(sprintf('\%s\%s','File ',filename))

if signalcheck $(\mathrm{i}, 1)==0$ ||

$\operatorname{sum}(\operatorname{signalcheck}(\mathrm{i}, 2: 1: 4))==0$

disp(sprintf(' $\%$ s\%s','Not enough data

for signal on file ',filename))

else

\%Load signal

clear subq1

clear subq2

clear subq3

clear screw

clear $\mathrm{t}$

load(filename);

clear $\mathrm{t}$

$\mathrm{t}=(1 / \mathrm{hz}: 1 / \mathrm{hz}:$ length $($ subq1 $) / \mathrm{hz})$;

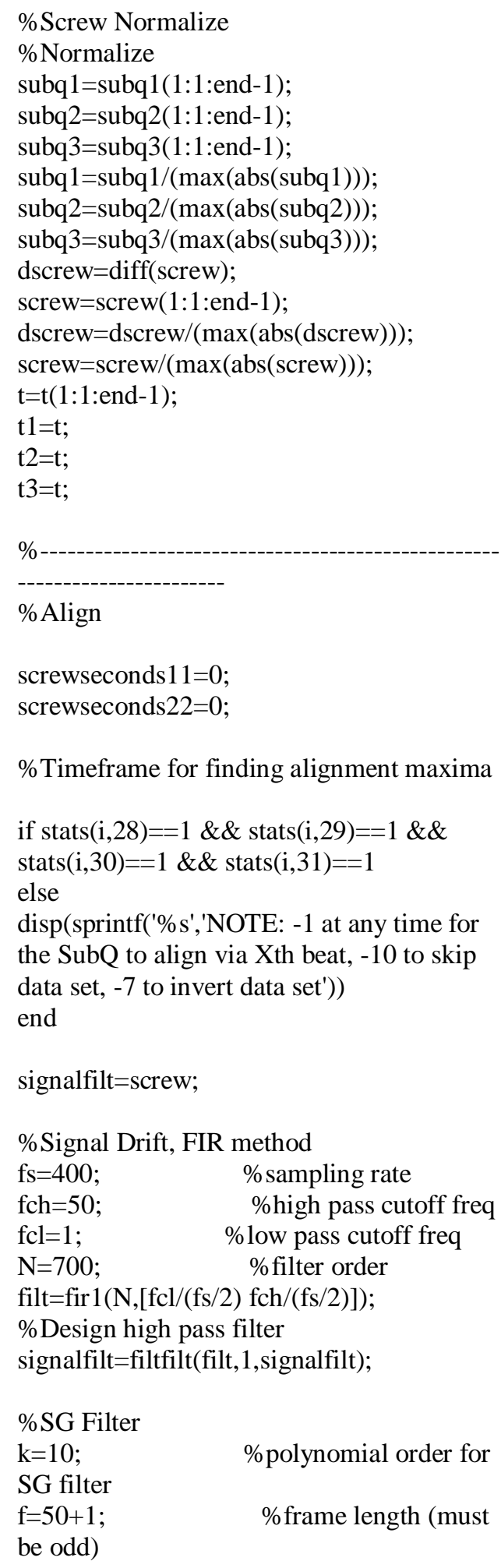

\%polynomial order for

$\%$ frame length (must 


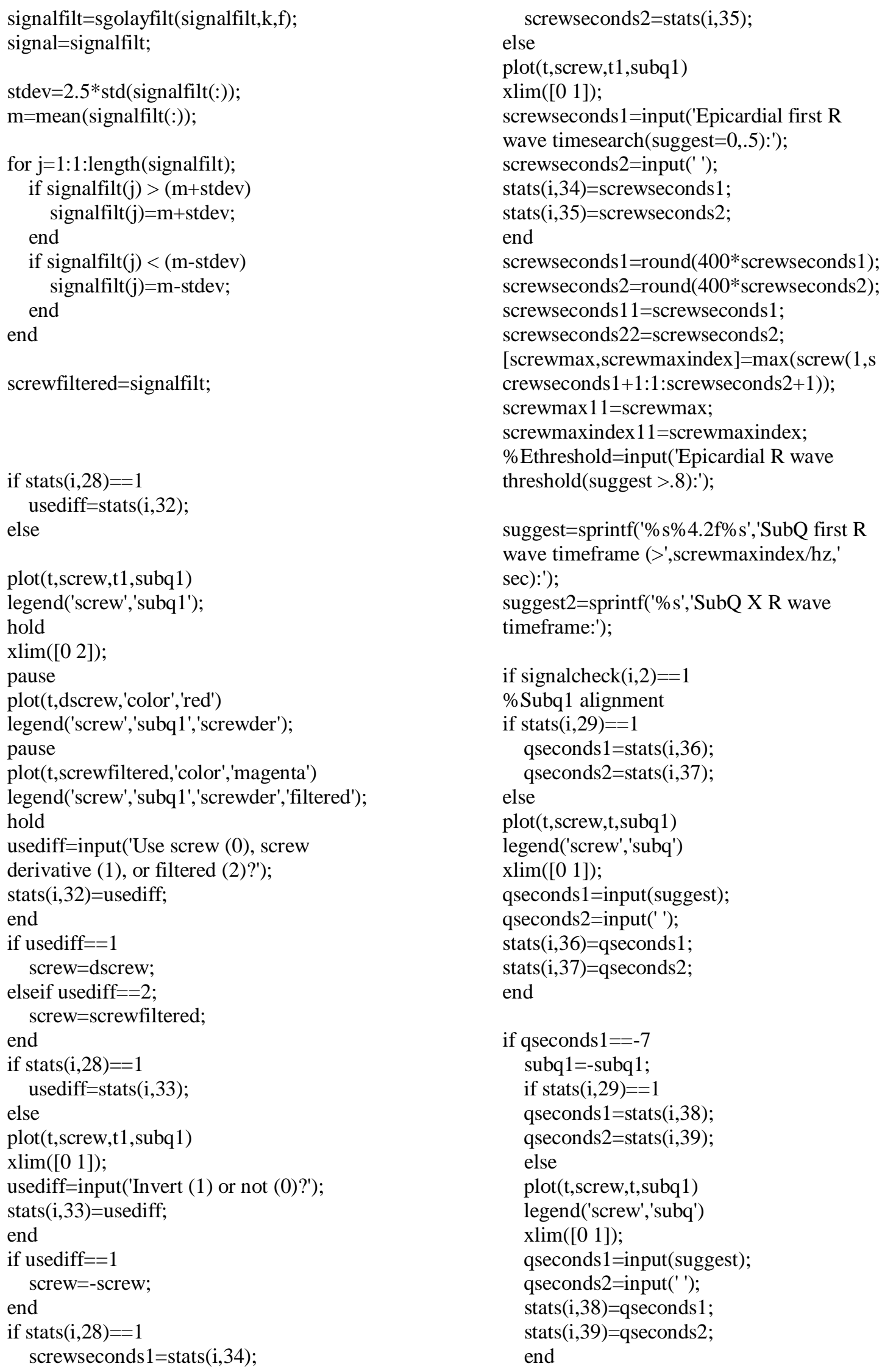

screwseconds $2=\operatorname{stats}(\mathrm{i}, 35)$;

else

$\operatorname{plot}(\mathrm{t}, \mathrm{screw}, \mathrm{t} 1, \mathrm{subq} 1)$

$x \lim \left(\left[\begin{array}{ll}0 & 1\end{array}\right]\right)$;

screwseconds1=input('Epicardial first R

wave timesearch(suggest $=0, .5): '$ );

screwseconds2=input(' ');

stats $(\mathrm{i}, 34)=$ screwseconds 1 ;

stats $(\mathrm{i}, 35)=$ screwseconds 2 ;

end

screwseconds $1=$ round $(400 *$ screwseconds 1$)$; screwseconds $2=$ round $(400 *$ screwseconds 2$)$; screwseconds $11=$ screwseconds 1 ; screwseconds $22=$ screwseconds 2 ;

[screwmax,screwmaxindex $]=\max (\operatorname{screw}(1, \mathrm{~s}$ crewseconds $1+1: 1$ :screwseconds $2+1)$ ); screwmax $11=$ screwmax; screwmaxindex 11=screwmaxindex; $\%$ Ethreshold=input('Epicardial R wave threshold(suggest >.8):');

suggest=sprintf('\%s\%4.2f\%s','SubQ first R wave timeframe (>',screwmaxindex/hz,' sec):');

suggest2=sprintf('\%s','SubQ X R wave timeframe:');

if $\operatorname{signalcheck}(\mathrm{i}, 2)==1$

$\%$ Subq1 alignment

if $\operatorname{stats}(\mathrm{i}, 29)==1$

qseconds $1=$ stats $(\mathrm{i}, 36)$;

qseconds $2=\operatorname{stats}(\mathrm{i}, 37)$;

else

plot(t,screw,t,subq1)

legend('screw','subq')

$x \lim \left(\left[\begin{array}{ll}0 & 1\end{array}\right]\right)$;

qseconds1=input(suggest);

qseconds $2=$ input(' ');

stats $(\mathrm{i}, 36)=$ qseconds 1 ;

stats $(\mathrm{i}, 37)=\mathrm{qseconds} 2$;

end

if qseconds $1==-7$

subq1=-subq1;

if $\operatorname{stats}(\mathrm{i}, 29)==1$

qseconds $1=$ stats $(\mathrm{i}, 38)$;

qseconds $2=\operatorname{stats}(\mathrm{i}, 39)$;

else

plot(t,screw,t,subq1)

legend('screw','subq')

$x \lim \left(\left[\begin{array}{ll}0 & 1\end{array}\right]\right)$;

qseconds1=input(suggest);

qseconds2=input(' ');

stats $(\mathrm{i}, 38)=$ qseconds 1 ;

stats $(\mathrm{i}, 39)=\mathrm{qseconds} 2$;

end 
end

if qseconds $1==-1$

if $\operatorname{stats}(\mathrm{i}, 29)==1$

screwseconds1=stats(i,38);

screwseconds $2=\operatorname{stats}(\mathrm{i}, 39)$;

else

plot(t,screw,t,subq1)

legend('screw','subq')

$x \lim \left(\left[\begin{array}{ll}0 & 2.5\end{array}\right]\right)$;

screwseconds1=input('Epicardial X R wave

timesearch:');

screwseconds2=input(' ');

stats $(\mathrm{i}, 38)=$ screwseconds 1 ;

stats $(\mathrm{i}, 39)=$ screwseconds 2 ;

end

screwseconds1=round(400*screwseconds1); screwseconds $2=$ round $(400 *$ screwseconds 2$)$; [screwmax, screwmaxindex] $=\max (\operatorname{screw}(1, \mathrm{~s}$ crewseconds 1+1:1:screwseconds2+1)); $\%$ Ethreshold=input('Epicardial R wave threshold(suggest $>.8$ ):');

if $\operatorname{stats}(\mathrm{i}, 29)==1$

qseconds $1=$ stats $(\mathrm{i}, 66)$;

qseconds $2=\operatorname{stats}(i, 67)$;

else

qseconds1=input(suggest2);

qseconds $2=$ input(' ');

stats $(\mathrm{i}, 66)=\mathrm{qseconds}$;

stats $(\mathrm{i}, 67)=\mathrm{qseconds} 2$; end

elseif qseconds $1==-10$

signalcheck $(\mathrm{i}, 2)=0$;

end

qseconds1=round(400*qseconds1); qseconds $2=$ round $(400 *$ qseconds 2$)$;

\%Qthreshold=input('SubQ R wave

threshold(suggest >.8):');

end

if $\operatorname{signalcheck}(\mathrm{i}, 2)==1$

$\% \%$ Search for $\mathrm{R}$ wave index

[qmax,qmaxindex] $=\max$ (subq1 $(1$, qseconds 1

$+1: 1$ :qseconds $2+1)$ );

QEdiff=(qmaxindex+qseconds1)-

(screwmaxindex+screwseconds1);

if QEdiff< 0 ;

if QEdiff $>-20$

else redo'));

disp(sprintf('\%s','Bad matchup please

end

else

subq1=subq1(1,QEdiff+1:1:end);

$\mathrm{t} 1=\mathrm{t}(1,1: 1$ :end-QEdiff); end

else

subq1=zeros(1,length(screw));

end

screwseconds $1=$ screwseconds 11 ;

screwseconds $2=$ screwseconds 22 ;

screwmax $=$ screwmax 11 ;

screwmaxindex $=$ screwmaxindex 11 ;

if signalcheck $(\mathrm{i}, 3)==1$

$\%$ Subq2 alignment

if $\operatorname{stats}(i, 30)==1$

qseconds $1=$ stats $(\mathrm{i}, 40)$;

qseconds $2=$ stats $(i, 41)$;

else

plot $(\mathrm{t}$, screw, $\mathrm{t}, \mathrm{subq} 2)$

legend('screw','subq')

$x \lim \left(\left[\begin{array}{ll}0 & 1\end{array}\right]\right)$;

qseconds1=input(suggest);

qseconds2=input(' ');

stats $(\mathrm{i}, 40)=$ qseconds 1 ;

stats $(i, 41)=$ qseconds 2 ;

end

if qseconds $1==-7$

subq2=-subq2;

if $\operatorname{stats}(\mathrm{i}, 30)==1$

qseconds $1=$ stats $(\mathrm{i}, 42)$;

qseconds $2=\operatorname{stats}(\mathrm{i}, 43)$;

else

$\operatorname{plot}(t, s c r e w, t, s u b q 2)$

legend('screw','subq')

$x \lim \left(\left[\begin{array}{ll}0 & 1\end{array}\right]\right)$;

qseconds1=input(suggest);

qseconds2=input(' ');

stats $(\mathrm{i}, 42)=$ qseconds 1 ;

stats $(\mathrm{i}, 43)=\mathrm{qseconds} 2$;

end

end

if qseconds $1==-1$

if $\operatorname{stats}(\mathrm{i}, 30)==1$

screwseconds $1=\operatorname{stats}(i, 42)$;

screwseconds $2=\operatorname{stats}(i, 43)$;

else

plot(t,screw,t,subq2)

legend('screw','subq')

$x \lim \left(\left[\begin{array}{ll}0 & 2.5\end{array}\right]\right)$;

screwseconds1=input('Epicardial X R wave timesearch:');

screwseconds2=input(' ');

screwseconds1=round $(400 *$ screwseconds 1$)$;

screwseconds $2=$ round $(400 *$ screwseconds 2$)$; stats $(\mathrm{i}, 42)=$ screwseconds 1 ; 


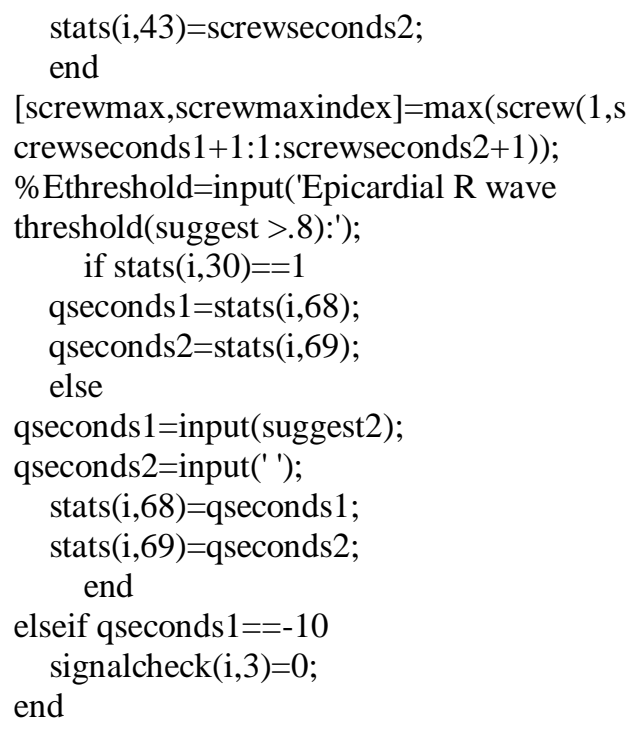

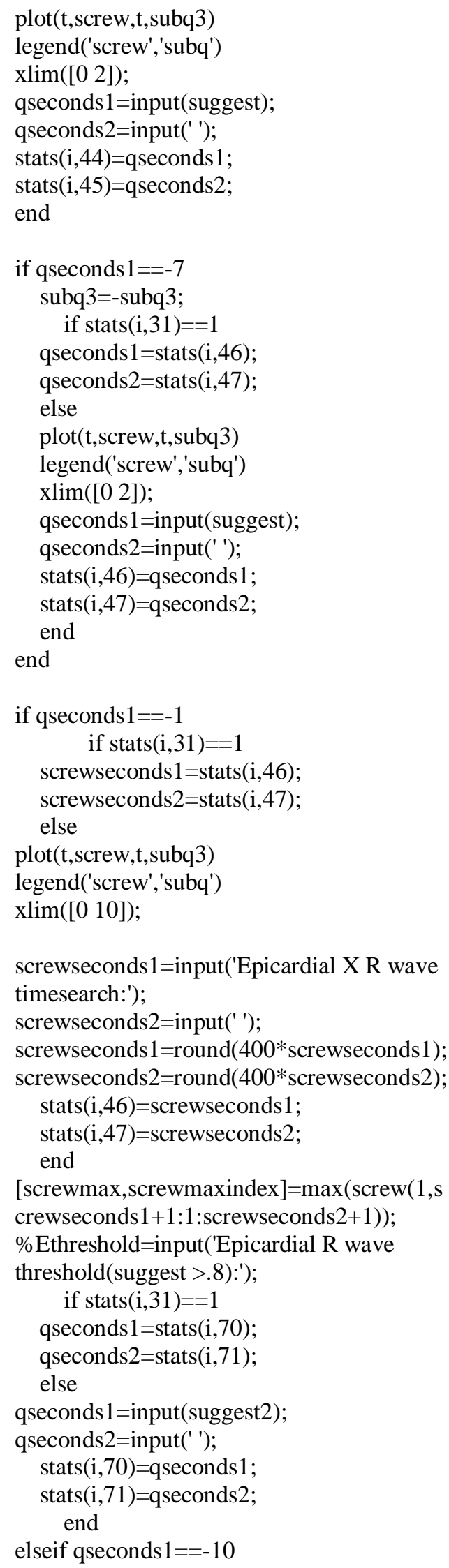




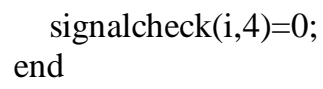

$\%$ Cut to same length cutmatrix $=[$ length $($ subq1 $)$,length $($ subq2), len gth(subq3),length(screw)]; screw $=\operatorname{screw}(1,1: 1: \min ($ cutmatrix $))$; subq1=subq1(1,1:1:length(screw $))$; subq $2=\operatorname{subq} 2(1,1: 1:$ length $($ screw $))$; subq3=subq3(1,1:1:length(screw $))$; $\mathrm{t}=\mathrm{t}(1,1: 1: \min ($ cutmatrix $))$;

$\mathrm{t} 1=\mathrm{t} 1(1,1: 1:$ length $(\mathrm{t}))$;

$\mathrm{t} 2=\mathrm{t} 2(1,1: 1:$ length $(\mathrm{t}))$

$\mathrm{t} 3=\mathrm{t} 3(1,1: 1:$ length $(\mathrm{t}))$;

\%Add blank period at end ( $2 \mathrm{sec}$ long) subq1(1,(length(subq1)+1): 1:(length(subq1) $+800))=\operatorname{zeros}(1,800)$; subq2(1,(length(subq2)+1): 1:(length(subq2) $+800))=\operatorname{zeros}(1,800)$; subq3(1,(length(subq3)+1): 1:(length(subq3) $+800))=$ zeros $(1,800)$;

screw(1,(length(screw)+1):1:(length(screw) $+800))=$ zeros $(1,800)$;

$\mathrm{t}(($ end +1$):($ end +800$))=(\mathrm{t}($ end $)+\mathrm{t}(2)):(\mathrm{t}(2)):(\mathrm{t}($ end $+\mathrm{t}(2) * 800)$;

$\mathrm{t} 1(($ end +1$):($ end +800$))=(\mathrm{t} 1($ end $)+\mathrm{t} 1(2)):(\mathrm{t} 1(2$ )$):(\mathrm{t} 1(\mathrm{end})+\mathrm{t} 1(2) * 800)$;

$\mathrm{t} 2(($ end +1$):($ end +800$))=(\mathrm{t} 2($ end $)+\mathrm{t} 2(2)):(\mathrm{t} 2(2$ )$):(\mathrm{t} 2(\mathrm{end})+\mathrm{t} 2(2) * 800)$;

$\mathrm{t} 3(($ end +1$):($ end +800$))=(\mathrm{t} 3($ end $)+\mathrm{t} 3(2)):(\mathrm{t} 3(2$ )$):(\mathrm{t} 3(\mathrm{end})+\mathrm{t} 3(2) * 800)$;

clear subq1o;

clear subq2o;

clear subq3o;

subq10=subq 1 ;

subq2o=subq2;

subq3o=subq3;

\%subq1

signalfilt=subq1;

\%Signal Drift, FIR method

fs $=400$

$\mathrm{fch}=50$

$\mathrm{fcl}=1$;

$\mathrm{N}=700$;

\%sampling rate \%high pass cutoff freq

filt=fir $1(\mathrm{~N},[\mathrm{fcl} /(\mathrm{fs} / 2) \mathrm{fch} /(\mathrm{fs} / 2)])$;

$\%$ Design high pass filter signalfilt=filtfilt(filt, 1 , signalfilt);

$\%$ SG Filter

$\mathrm{k}=10$;

SG filter

$\mathrm{f}=50+1$; $\quad \%$ frame length (must

be odd)

signalfilt=sgolayfilt(signalfilt, $\mathrm{k}, \mathrm{f})$;

signal=signalfilt;

stdev $=2.5^{*} \operatorname{std}($ signalfilt $(:))$;

$\mathrm{m}=$ mean $($ signalfilt(:));

for $\mathrm{j}=1: 1$ :length(signalfilt);

if signalfilt $(j)>(m+$ stdev $)$ signalfilt $(j)=m+$ stdev;

end

if signalfilt $(\mathrm{j})<(\mathrm{m}$-stdev) end signalfilt $(j)=m-s t d e v$;

end

subq1=signalfilt;

$\%$ subq2 
signalfilt=subq2;

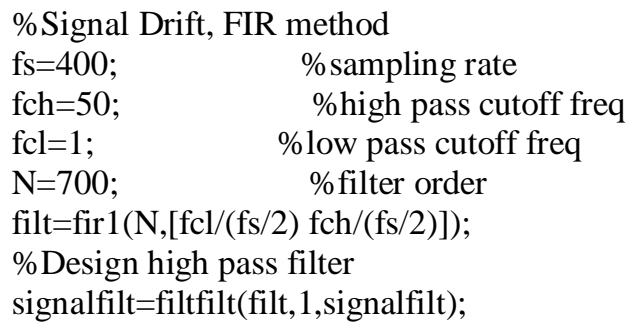

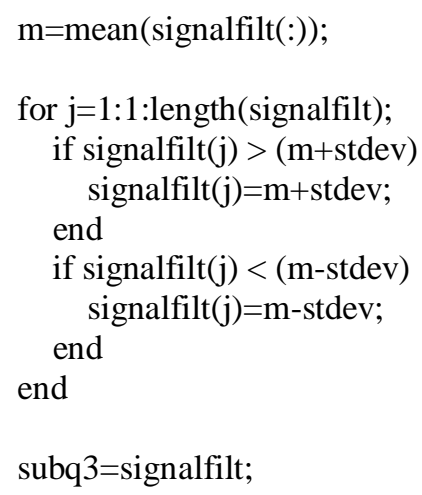

if $\operatorname{stats}(\mathrm{i}, 28)==1 \& \&$ stats $(\mathrm{i}, 29)==1 \& \&$ $\operatorname{stats}(\mathrm{i}, 30)==1 \quad \& \& \operatorname{stats}(\mathrm{i}, 31)==1$

else

plot(t,screw,t1,subq1,t2,subq2,t3,subq3)

legend('screw','subq1','subq2','subq3')

$x \lim \left(\left[\begin{array}{ll}0 & 10\end{array}\right)\right.$

pause

end

\% Threshold Detection Program

resolution $=.17 * 400 ; \quad \%$ Detection

Resolution

\%screw R wave detection

clear signalfilt;

clear trigger;

clear ts;

$\mathrm{ts}=\mathrm{t}$;

signalfilt=screw;

if $\operatorname{stats}(i, 28)==1$

ithresh $=$ stats $(\mathrm{i}, 48)$;

iblank $=$ stats $(\mathrm{i}, 49)$;

spblank=stats $(\mathrm{i}, 50)$;

else

plot $(\mathrm{t}, \mathrm{screw})$;

$x \lim \left(\left[\begin{array}{ll}0 & 2.5\end{array}\right]\right)$ 
ithresh=input('First $3 \mathrm{R}$ wave detection threshold:');

iblank=input('Initial blanking period in sec:');

spblank=input('Ignore period in sec:'); stats $(\mathrm{i}, 48)=\mathrm{ithresh}$; stats $(\mathrm{i}, 49)=\mathrm{iblank}$;

stats $(\mathrm{i}, 50)=$ spblank;

end

iblank=iblank $* 400$;

RRT1 $=0$;

RRT2 $=0$;

RRT3=0;

RRV1=0;

RRV2=0;

RRV3=0;

blankpercent $=.80$;

initial $\mathrm{R}=1$

initialrsec $=.050$;

ramppercent $=.80$;

trigger $=$ zeros $(1$, length $($ screw $))$;

lastR $=0$;

RRTvariabilityfactor $=.3$;

RRVvariabilityfactor $=.3$;

blankpercent $=.80$;

initial $\mathrm{R}=1$;

ppercent $=.35$;

tpercent $=.50$;

rpercentforward $=.10$;

rpercentbackward $=.05$;

initialrsec $=.050$;

ramppercent $=.80$;

lastR $=0$;

RRTvariabilityfactor $=.3$;

RRVvariabilityfactor $=.3$;

clear pstats

clear rstats

clear tstats

$\mathrm{pz}=1$;

$\mathrm{rZ}=1$

$\mathrm{tz}=1$;

for $\mathrm{k}=\left(1+\right.$ spblank $\left.^{*} 400\right): 1$ :(length( signalfilt $)$ 800);

if initialR $==3$

if signalfilt $(\mathrm{k})>=\mathrm{ithresh} \& \&(\mathrm{k}-$

RRT1)>iblank

trigger $(\mathrm{k})=1$;

RRT3=RRT2;

RRT2=RRT1;

RRT1=k;

lastR=k;

RRV3=RRV2;

RRV2=RRV1;
RRV1=max $($ signalfilt $(\mathrm{k}:(\mathrm{k}+$ initialrsec $* 400))$

);

initialR=initialR +1 ;

RRT3=RRT2-RRT3;

RRT2=RRT1-RRT2;

RRT1=RRT2;

end

elseif initialR $==2$

if signalfilt $(\mathrm{k})>=$ ithresh $\& \&(\mathrm{k}-$

RRT1)>iblank

trigger $(\mathrm{k})=1$;

RRT3=RRT2;

RRT2=RRT1;

RRT1=k;

RRV3=RRV2;

RRV2=RRV1;

RRV1=max $($ signalfilt $(\mathrm{k}:(\mathrm{k}+$ initialrsec $* 400))$

);

initialR=initialR +1 ;

end

elseif initialR $==1$

if signalfilt $(\mathrm{k})>=\mathrm{ithresh}$

trigger $(\mathrm{k})=1$;

RRT3=RRT2;

RRT2=RRT1;

RRT1=k;

RRV3=RRV2;

RRV2=RRV1;

RRV1=max (signalfilt $(\mathrm{k}:(\mathrm{k}+$ initialrsec*400) $)$

);

initialR=initialR +1 ;

end

else

if signalfilt $(\mathrm{k})>=\mathrm{ithresh} \& \&(\mathrm{k}-$

lastR $)>(((\mathrm{RRT} 1+\mathrm{RRT} 2+\mathrm{RRT} 3) / 3) *$ blankper cent)

trigger $(\mathrm{k})=1$;

RRT3=RRT2;

RRT2=RRT1;

if $(\mathrm{k}-$

lastR $)>((\mathrm{RRT} 1+\mathrm{RRT} 2+\mathrm{RRT} 3) / 3) *(1+\mathrm{RRTV}$

ariabilityfactor) $\|(\mathrm{k}-$

lastR $)<((\mathrm{RRT} 1+\mathrm{RRT} 2+\mathrm{RRT} 3) / 3) *(1-$

RRTvariabilityfactor)

else

RRT1=k-lastR;

$\% \quad \%$ p,t,r wave capture and voltage

indexing

$\% \quad$ pwave(round(k-(k-

lastR)*(ppercent+rpercentbackward)):round( $\mathrm{k}-(\mathrm{k}-$

lastR)*rpercentbackward $))=\mathrm{ECG}($ round $(\mathrm{k}-$

(k- 
lastR)*(ppercent+rpercentbackward)):round( k-(k-lastR)*rpercentbackward));

$\%$

pstats $(1, \mathrm{pz})=\mathrm{abs}(\max ($ pwave $($ round $(\mathrm{k}-(\mathrm{k}-$ lastR)*(ppercent+rpercentbackward)):round( $\mathrm{k}-(\mathrm{k}-$ lastR)*rpercentbackward $)))$ -

$\min$ (pwave(round(k-(k-

lastR)*(ppercent+rpercentbackward)):round( $\mathrm{k}-(\mathrm{k}-\mathrm{lastR}) *$ rpercentbackward $)))$ ); $\% \quad$ twave(round(k-(k-lastR)*(1rpercentforward)):round(k-(k-lastR)*(1rpercentforward-tpercent $))=E C G(\operatorname{round}(k-$ (k-lastR)*(1-rpercentforward)):round(k-(klastR)*(1-rpercentforward-tpercent))); $\%$

tstats $(1, \mathrm{tz})=\mathrm{abs}(\max (\mathrm{twave}(\mathrm{round}(\mathrm{k}-(\mathrm{k}-$ lastR)*(1-rpercentforward)):round(k-(klastR $*(1$-rpercentforward-tpercent $))))$ min(twave(round(k-(k-lastR)*(1rpercentforward)):round(k-(k-lastR)*(1rpercentforward-tpercent)))));

$\%$ rwave(round(k-(klastR)*(rpercentbackward $))$ :round $(\mathrm{k}+(\mathrm{k}-$ lastR $) *($ rpercentforward $))=\mathrm{ECG}($ round $(\mathrm{k}-$ (k-lastR)*(rpercentbackward $))$ :round $(\mathrm{k}+(\mathrm{k}-$ lastR)*(rpercentforward))); $\%$ rstats $(1, \mathrm{rz})=\mathrm{abs}(\max ($ rwave $(\operatorname{round}(\mathrm{k}-(\mathrm{k}-$ lastR)*(rpercentbackward $))$ :round $(\mathrm{k}+(\mathrm{k}-$ lastR)*(rpercentforward $)))$ )-

$\min$ (rwave(round(k-(k-

lastR $) *($ rpercentbackward $))$ :round $(\mathrm{k}+(\mathrm{k}-$

lastR)*(rpercentforward)))));

$\% \quad \%$ p,t wave / $\mathrm{r}$ wave ratio

indexing

$\%$

pstats $(2, \mathrm{pz})=\operatorname{pstats}(1, \mathrm{pz}) / \mathrm{rstats}(1, \mathrm{rz})$;

$\%$

$\operatorname{tstats}(2, \mathrm{tz})=\operatorname{tstats}(1, \mathrm{tz}) / \mathrm{rstats}(1, \mathrm{rz})$;

$\%$

[maxv,prt1] $=\max ($ rwave $($ round $(k-(k-$ lastR)*(rpercentbackward $))$ :round $(\mathrm{k}+(\mathrm{k}-$ lastR)*(rpercentforward))));

$\%$

$[\operatorname{maxv}, \operatorname{prt} 2]=\max (\operatorname{abs}(\operatorname{diff}($ pwave $($ round $(\mathrm{k}-$

(k-

lastR)*(ppercent+rpercentbackward)):round( k-(k-lastR)*rpercentbackward)))));

$\% \quad \operatorname{pstats}(3, \mathrm{pz})=(\operatorname{prt} 1+\operatorname{round}(\mathrm{k}-(\mathrm{k}-$ lastR)*(rpercentbackward))-prt2-round(k-(klastR $)^{*}($ ppercent+rpercentbackward $\left.\left.)\right)\right) / 400$; $\%$

[maxv,trt2] $=\max (\operatorname{abs}(\operatorname{diff}(\mathrm{twave})($ round $(\mathrm{k}-(\mathrm{k}-$ lastR)*(1-rpercentforward)):round(k-(klastR)*(1-rpercentforward-tpercent))))));

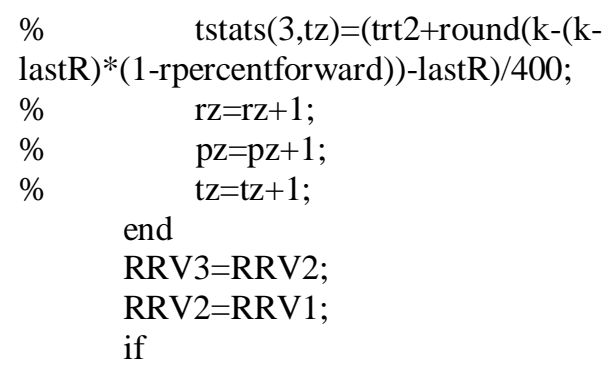

clear twave

clear pwave

clear rwave

twave=zeros $(1$,length $(\mathrm{t} 1))$; 
RRV1=max (signalfilt $(\mathrm{k}:(\mathrm{k}+$ initialrsec*400))

);

initialR=initialR+1;

end

elseif initialR $==1$

if signalfilt $(\mathrm{k})>=$ ithresh

trigger $(\mathrm{k})=1$;

RRT3=RRT2;

RRT2=RRT1;

RRT1=k;

RRV3=RRV2;

RRV2=RRV1;

RRV1=max $($ signalfilt $(\mathrm{k}:(\mathrm{k}+$ initialrsec*400) $)$

);

initialR=initialR +1 ;

$$
\text { end }
$$

else

if

signalfilt $(\mathrm{k})>=((\mathrm{RRV} 1+\mathrm{RRV} 2+\mathrm{RRV} 3) / 3 * \mathrm{ra}$

mppercent) \&\& (k-

lastR $)>(((\mathrm{RRT} 1+\mathrm{RRT} 2+\mathrm{RRT} 3) / 3) *$ blankper cent)

trigger $(\mathrm{k})=1$;

if $(\mathrm{k}-$

lastR $)>((\mathrm{RRT} 1+\mathrm{RRT} 2+\mathrm{RRT} 3) / 3) *(1+\mathrm{RRTv}$

ariabilityfactor) $\|(\mathrm{k}-$

lastR $)<((\mathrm{RRT} 1+\mathrm{RRT} 2+\mathrm{RRT} 3) / 3) *(1-$

RRTvariabilityfactor)

pstats $(1: 4, p z)=0$;

$\operatorname{tstats}(1: 4, \mathrm{tz})=0$;

$\operatorname{rstats}(1: 4, \mathrm{rz})=0$;

$\mathrm{rz}=\mathrm{rz}+1$;

$\mathrm{pz}=\mathrm{pz}+1$;

$\mathrm{tz}=\mathrm{tz}+1$

else

RRT2=RRT1;

RRT3=RRT2;

RRT1=k-lastR;

indexing

$\%$ p,t,r wave capture and voltage

pwave(round(k-(k-

lastR)*(ppercent+rpercentbackward)):round( $\mathrm{k}-(\mathrm{k}-$

lastR)*rpercentbackward))=ECG(round(k-

(k-

lastR)*(ppercent+rpercentbackward)):round( k-(k-lastR)*rpercentbackward));

pstats $(1, \mathrm{pz})=\mathrm{abs}(\max ($ pwave $($ round $(\mathrm{k}-(\mathrm{k}-$

lastR)*(ppercent+rpercentbackward)):round(

$\mathrm{k}$-(k-lastR)*rpercentbackward)))-

$\min$ (pwave(round (k-(k-

lastR)*(ppercent+rpercentbackward)):round( k-(k-lastR)*rpercentbackward))));
twave(round(k-(k-lastR)*(1rpercentforward)):round(k-(k-lastR)*(1rpercentforward-tpercent $)))=E C G($ round $(k-$ (k-lastR)*(1-rpercentforward)):round(k-(klastR)*(1-rpercentforward-tpercent)));

tstats $(1, \mathrm{tz})=\mathrm{abs}(\max (\mathrm{twave}(\mathrm{round}) \mathrm{k}-(\mathrm{k}-$ lastR)*(1-rpercentforward)):round(k-(klastR)*(1-rpercentforward-tpercent))))$\min ($ twave(round(k-(k-lastR)*(1rpercentforward)):round(k-(k-lastR)*(1rpercentforward-tpercent))))); rwave(round $(\mathrm{k}-(\mathrm{k}-$

lastR)*(rpercentbackward $))$ :round $(\mathrm{k}+(\mathrm{k}-$ lastR $) *($ rpercentforward $))=E C G($ round $(\mathrm{k}-$ $(\mathrm{k}-\mathrm{lastR}) *($ rpercentbackward $))$ :round(k+(klastR)*(rpercentforward)));

rstats $(1, \mathrm{rz})=\mathrm{abs}(\max (\mathrm{rwave})$ round $(\mathrm{k}-(\mathrm{k}-$ lastR $) *($ rpercentbackward $))$ :round $(\mathrm{k}+(\mathrm{k}-$ lastR)*(rpercentforward))))-

$\min$ (rwave(round $(\mathrm{k}-(\mathrm{k}-$

lastR)*(rpercentbackward)):round(k+(klastR)*(rpercentforward $))))$ ); $\% \mathrm{p}, \mathrm{t}$ wave / $\mathrm{r}$ wave ratio indexing

pstats $(2, p z)=p s t a t s(1, p z) /$ rstats $(1$, rz $)$; $\operatorname{tstats}(2, \mathrm{tz})=\operatorname{tstats}(1, \mathrm{tz}) / \mathrm{rstats}(1, \mathrm{rz})$; [maxv,prt1] $=\max ($ rwave $($ round $(\mathrm{k}-$

$(\mathrm{k}-\mathrm{lastR}) *($ rpercentbackward $))$ :round(k+(klastR)*(rpercentforward)))); if ipwave $==1$

[maxv,prt2] $=\min (($ pwave $($ round $(\mathrm{k}-(\mathrm{k}-$ lastR)*(ppercent+rpercentbackward)):round( k-(k-lastR)*rpercentbackward)))); else

[maxv,prt2] $=\max (($ pwave $($ round $(\mathrm{k}-(\mathrm{k}-$ lastR)*(ppercent+rpercentbackward)):round( k-(k-lastR)*rpercentbackward)))); end pstats $(3, \mathrm{pz})=(\mathrm{prt} 1+\operatorname{round}(\mathrm{k}-(\mathrm{k}-$

lastR)*(rpercentbackward))-prt2-round(k-(klastR)*(ppercent+rpercentbackward)))/400; if $\mathrm{pz}>1$; if $\operatorname{pstats}(1: 4, p z-1)==0$

pstats $(4, \mathrm{pz})=(\mathrm{prt} 2+\mathrm{round}(\mathrm{k}-(\mathrm{k}-$ lastR)*(ppercent+rpercentbackward)))/400; psave $=$ pstats $(4, p z)$; else

(k$\operatorname{pstats}(4, \mathrm{pz})=(\operatorname{prt} 2+\operatorname{round}(\mathrm{k}-$

lastR)*(ppercent+rpercentbackward)))/400psave; 


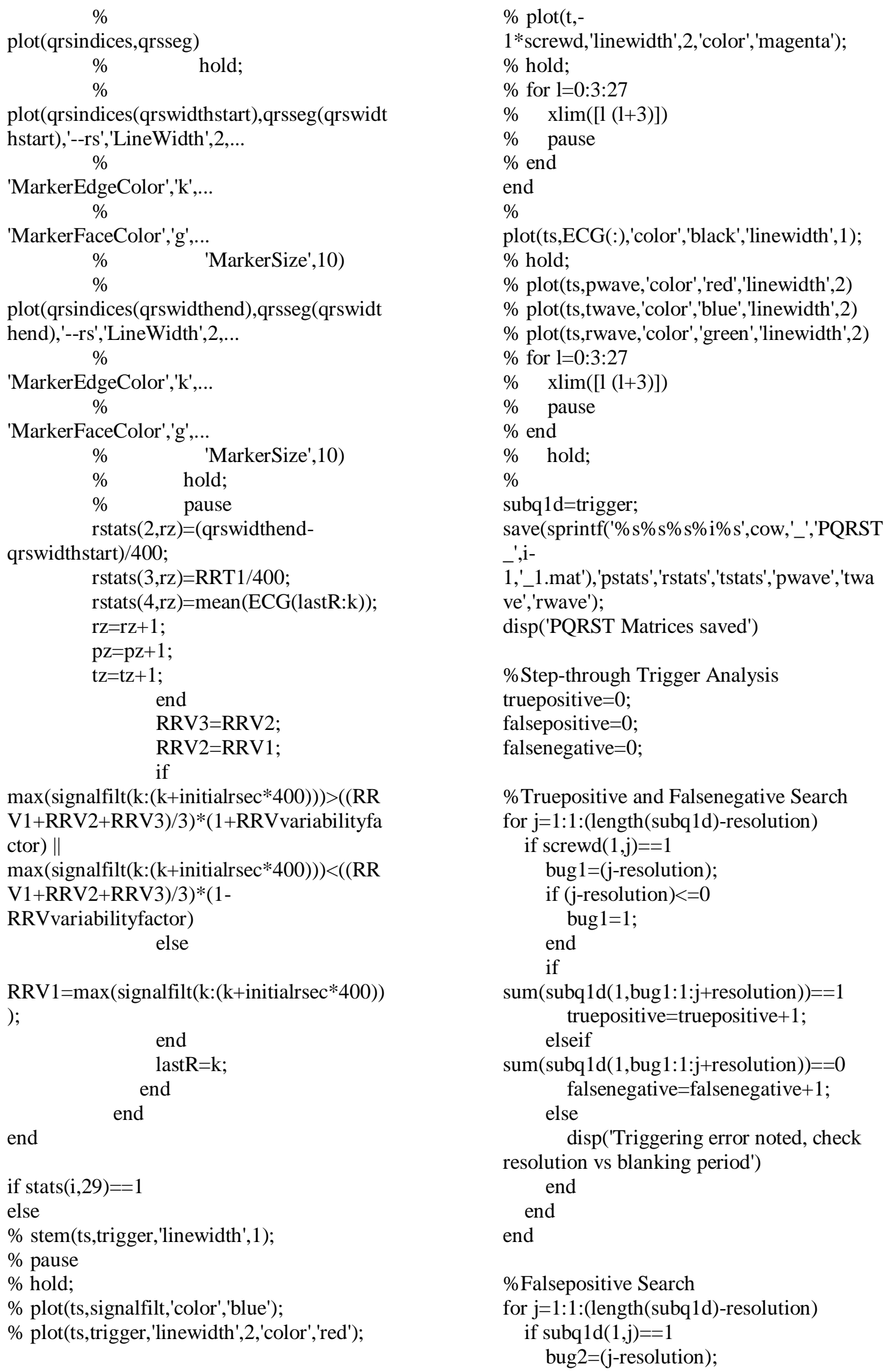

$\%$

plot(qrsindices,qrsseg)

$\%$ hold;

$\%$

plot(qrsindices(qrswidthstart), qrsseg(qrswidt

hstart),'--rs','LineWidth',2,... $\%$

'MarkerEdgeColor','k',...

$\%$

'MarkerFaceColor', 'g',... $\%$ 'MarkerSize',10)

$\%$

plot(qrsindices(qrswidthend), qrsseg(qrswidt hend),'--rs','LineWidth',2,... $\%$

'MarkerEdgeColor','k',... $\%$

'MarkerFaceColor', 'g',...

$\begin{array}{ll}\% & \text { 'MarkerSize',10) } \\ \% & \text { hold; } \\ \% & \text { pause } \\ \text { rstats(2,rz) }=(\text { qrswidthend- }\end{array}$

qrswidthstart)/400; rstats $(3, \mathrm{rz})=\mathrm{RRT} 1 / 400$; $\operatorname{rstats}(4, \mathrm{rz})=$ mean $(\mathrm{ECG}($ lastR:k)$)$;

$\mathrm{rz}=\mathrm{rz}+1$;

$\mathrm{pz}=\mathrm{pz}+1$;

$\mathrm{tz}=\mathrm{tz}+1$;

end

RRV3=RRV2;

RRV2=RRV1;

if

$\max ($ signalfilt $(\mathrm{k}:(\mathrm{k}+$ initialrsec*400) $))>((\mathrm{RR}$

V1+RRV2+RRV3)/3)*(1+RRVvariabilityfa ctor) $\|$

$\max ($ signalfilt $(\mathrm{k}:(\mathrm{k}+$ initialrsec $* 400)))<((\mathrm{RR}$

V1+RRV2+RRV3)/3)*(1-

RRVvariabilityfactor)

else

RRV1=max $($ signalfilt $(\mathrm{k}:(\mathrm{k}+$ initialrsec $* 400))$ );

$$
\begin{aligned}
& \text { end } \\
& \text { lastR=k; } \\
& \text { end } \\
& \text { end }
\end{aligned}
$$

end

if $\operatorname{stats}(\mathrm{i}, 29)==1$

else

$\%$ stem(ts,trigger,'linewidth', 1);

$\%$ pause

$\%$ hold;

$\%$ plot(ts,signalfilt,'color','blue');

$\%$ plot(ts,trigger,'linewidth',2,'color','red'); 


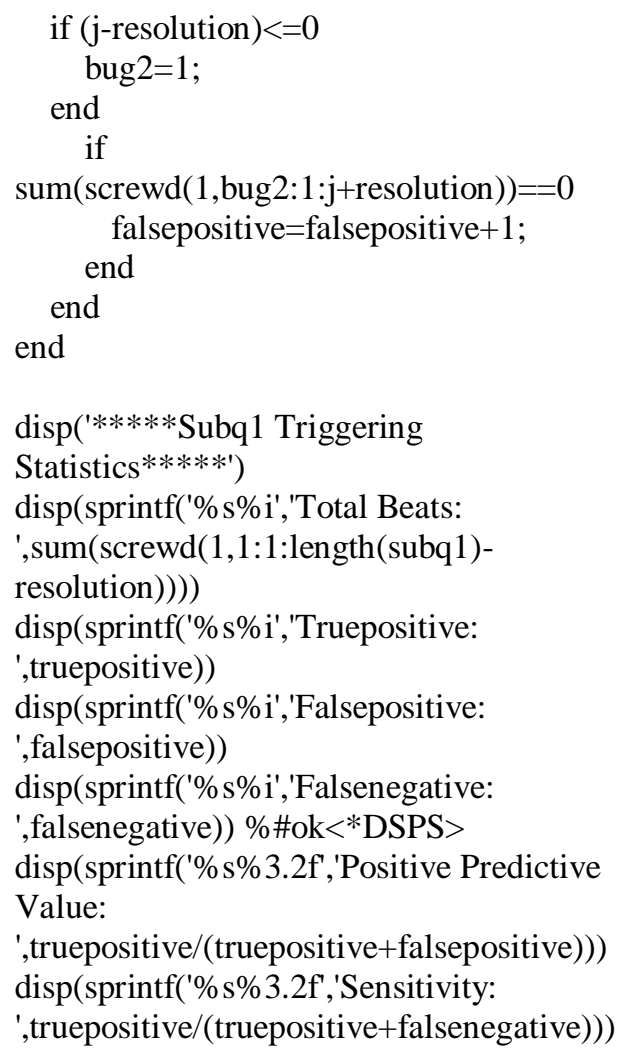

stats $(\mathrm{i}, 10: 15)=[\operatorname{sum}(\operatorname{screwd}(1,1: 1:$ length $($ sub q1d)-resolution))

clear twave clear pwave clear rwave

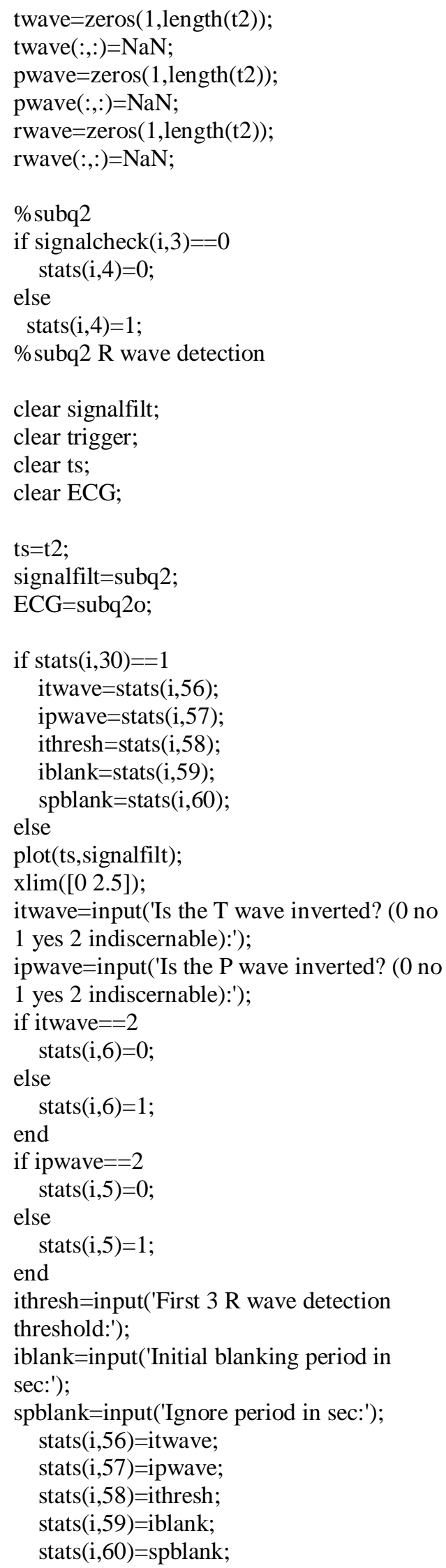




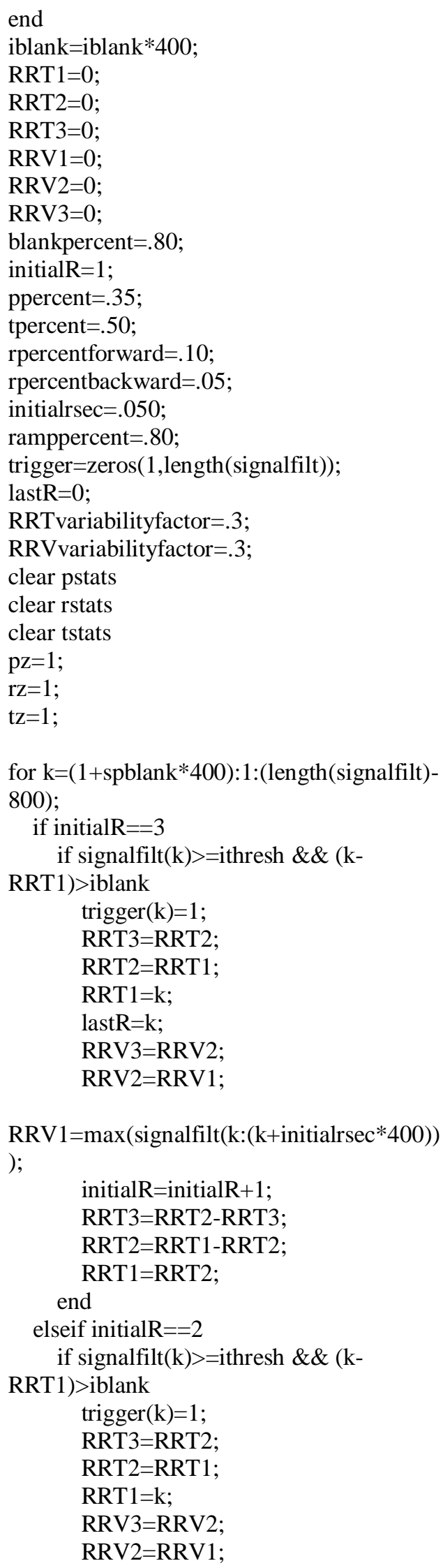


twave(round(k-(k-lastR)*(1rpercentforward)):round(k-(k-lastR)*(1rpercentforward-tpercent $))=E C G(\operatorname{round}(k-$ (k-lastR)*(1-rpercentforward)):round(k-(klastR)*(1-rpercentforward-tpercent)));

tstats $(1, \mathrm{tz})=\mathrm{abs}(\max ($ twave $($ round $(\mathrm{k}-(\mathrm{k}-$ lastR)*(1-rpercentforward)):round(k-(klastR $*(1$-rpercentforward-tpercent $))))$ $\min ($ twave(round(k-(k-lastR)*(1rpercentforward)):round(k-(k-lastR)*(1rpercentforward-tpercent))))); rwave(round $(\mathrm{k}-(\mathrm{k}-$ lastR)*(rpercentbackward $))$ :round $(\mathrm{k}+(\mathrm{k}-$ lastR $) *($ rpercentforward $))=E C G($ round $(\mathrm{k}-$ $(\mathrm{k}-$ lastR $) *($ rpercentbackward $))$ :round $(\mathrm{k}+(\mathrm{k}-$ lastR)*(rpercentforward)));

rstats $(1, \mathrm{rz})=\mathrm{abs}(\max ($ rwave $(\operatorname{round}(\mathrm{k}-(\mathrm{k}-$ lastR)*(rpercentbackward $))$ :round $(\mathrm{k}+(\mathrm{k}-$ lastR)*(rpercentforward $)))$ )$\min$ (rwave(round(k-(klastR)*(rpercentbackward $))$ :round $(\mathrm{k}+(\mathrm{k}-$ lastR)*(rpercentforward))))); $\% \mathrm{p}, \mathrm{t}$ wave / $\mathrm{r}$ wave ratio indexing

$\operatorname{pstats}(2, \mathrm{pz})=\operatorname{pstats}(1, \mathrm{pz}) / \mathrm{rstats}(1, \mathrm{rz})$; tstats $(2, \mathrm{tz})=\operatorname{tstats}(1, \mathrm{tz}) / \mathrm{rstats}(1, \mathrm{rz})$; $[\operatorname{maxv}, \operatorname{prt} 1]=\max (\operatorname{rwave}(\operatorname{round}(\mathrm{k}-$ (k-lastR)*(rpercentbackward $))$ :round $(\mathrm{k}+(\mathrm{k}-$ lastR)*(rpercentforward)))); if ipwave $==1$

[maxv,prt2] $=\min (($ pwave $($ round $(\mathrm{k}-(\mathrm{k}-$ lastR)*(ppercent+rpercentbackward)):round( $\mathrm{k}$-(k-lastR)*rpercentbackward)))); else

[maxv,prt2] $=\max (($ pwave $($ round $(\mathrm{k}-(\mathrm{k}-$ lastR)*(ppercent+rpercentbackward)):round( $\mathrm{k}-(\mathrm{k}-\mathrm{lastR}) *$ rpercentbackward)))); end pstats $(3, \mathrm{pz})=(\operatorname{prt} 1+\operatorname{round}(\mathrm{k}-(\mathrm{k}-$ lastR)*(rpercentbackward))-prt2-round(k-(klastR $)^{*}($ ppercent+rpercentbackward $\left.\left.)\right)\right) / 400$; if $\mathrm{pz}>1$; if $\operatorname{pstats}(1: 4, \mathrm{pz}-1)==0$

$\operatorname{pstats}(4, \mathrm{pz})=(\mathrm{prt} 2+\operatorname{round}(\mathrm{k}-(\mathrm{k}-$ lastR)*(ppercent+rpercentbackward)))/400; psave $=$ pstats $(4, p z)$; else

$(\mathrm{k}-$ $\operatorname{pstats}(4, \mathrm{pz})=(\operatorname{prt} 2+\operatorname{round}(\mathrm{k}-$

lastR)*(ppercent+rpercentbackward)))/400psave;

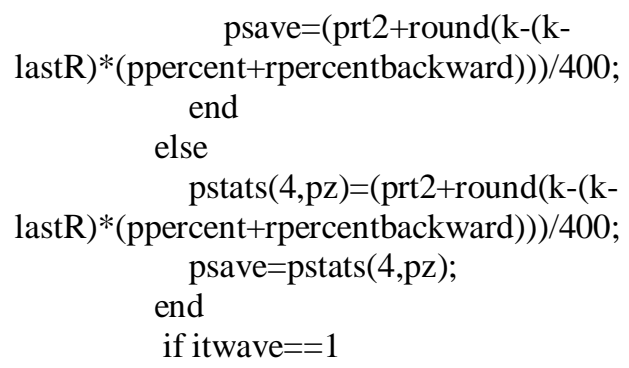

[maxv,trt2] $=\min ($ (twave $($ round $(\mathrm{k}-(\mathrm{k}-$ lastR)*(1-rpercentforward)):round(k-(klastR)*(1-rpercentforward-tpercent))))); else

$[\mathrm{max}, \mathrm{trt} 2]=\max ((\mathrm{twave})($ round $(\mathrm{k}-(\mathrm{k}-$ lastR)*(1-rpercentforward)):round(k-(klastR)*(1-rpercentforward-tpercent))))); end tstats $(3, \mathrm{tz})=(\operatorname{trt} 2+\operatorname{round}(\mathrm{k}-(\mathrm{k}-$

lastR)*(1-rpercentforward))-lastR)/400; if $\mathrm{tz}>1$; if $\operatorname{tstats}(1: 4, \mathrm{tz}-1)==0$

tstats $(4, \mathrm{tz})=(\operatorname{trt} 2+\operatorname{round}(\mathrm{k}-(\mathrm{k}-\mathrm{lastR}) *(1-$ rpercentforward)))/400;

tsave $=$ tstats $(4, \mathrm{tz})$

else tstats $(4, \mathrm{tz})=(\operatorname{trt} 2+\operatorname{round}(\mathrm{k}-(\mathrm{k}-$

lastR)*(1-rpercentforward)))/400-tsave; tsave $=(\operatorname{trt} 2+\operatorname{round}(\mathrm{k}-(\mathrm{k}-$

lastR)*(1-rpercentforward)))/400; end

else $\operatorname{tstats}(4, \mathrm{tz})=(\operatorname{trt} 2+\operatorname{round}(\mathrm{k}-(\mathrm{k}-$

lastR)*(1-rpercentforward)))/400; tsave $=\operatorname{tstats}(4, \mathrm{tz})$; end \%qrslength routine rmstart=round $(\mathrm{k}-(\mathrm{k}-$

lastR)*(rpercentbackward)); rmend $=$ round $(\mathrm{k}+(\mathrm{k}-$

lastR)*(rpercentforward)); qrsseg=ECG(1,rmstart:rmend); qrsseg $=$ qrsseg $+(0-\min ($ qrsseg $))$; qrsseg=qrsseg/max(qrsseg); [qrsmax

ignoreindex $]=\max ($ qrsseg$)$; averaging $=6$; qrswidthstart $=0$; qrsdiffpercent=.8; for $\mathrm{jkl}=$ averaging:-1:1 clear averagingm iii $=0$; if qrswidthstart $==0$ for $\mathrm{ii}=($ ignoreindex $):-1: 1$; 


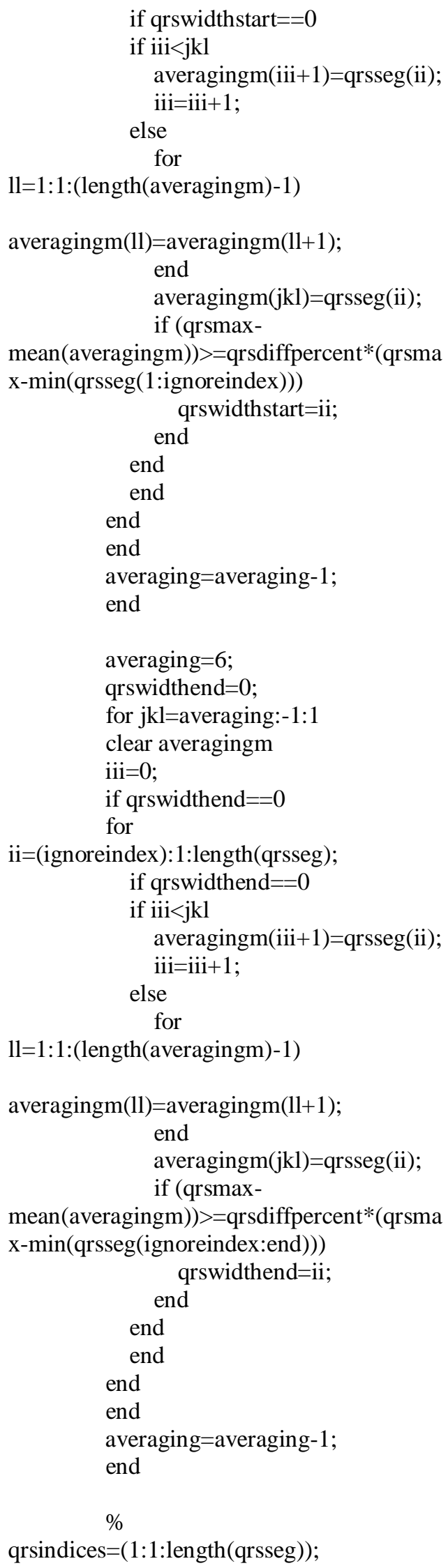

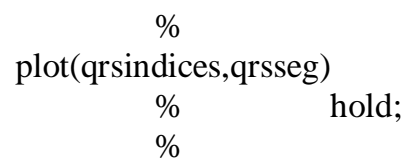




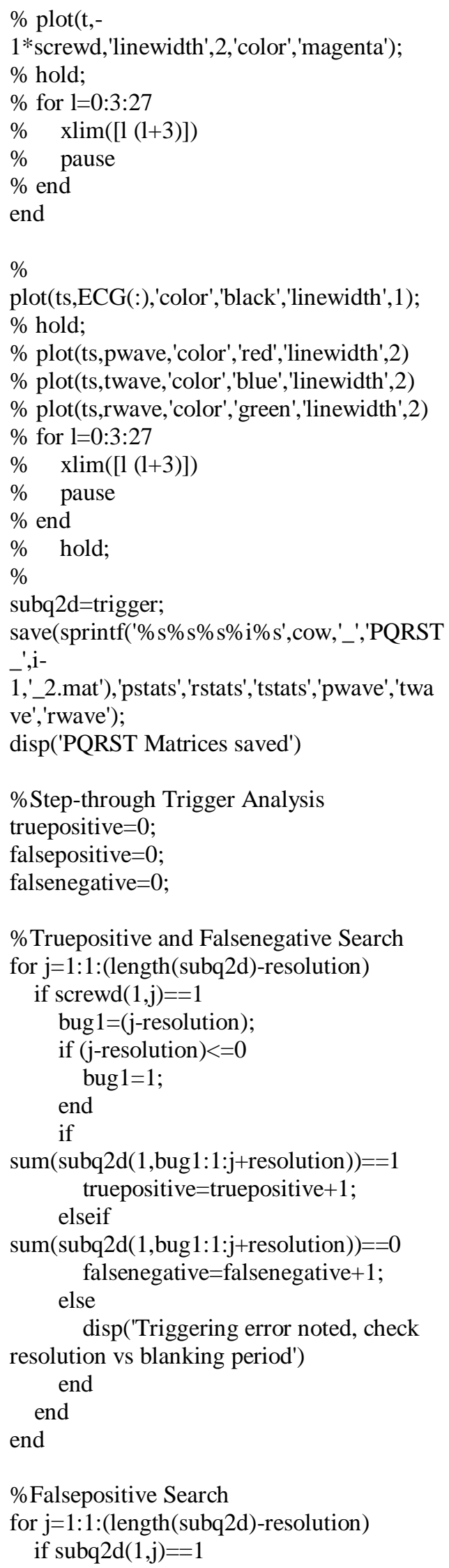




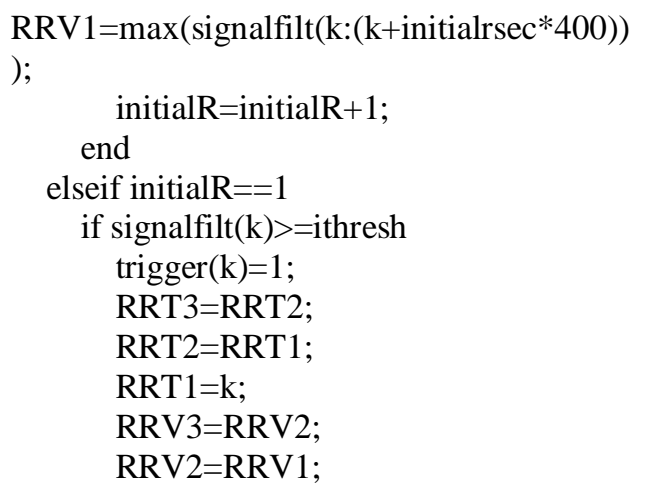

RRV1=max $($ signalfilt $(\mathrm{k}:(\mathrm{k}+$ initialrsec $* 400))$

) ;

$$
\begin{aligned}
& \text { initial } R=\text { initial } R+1 \text {; } \\
& \text { end }
\end{aligned}
$$

else

if

signalfilt $(\mathrm{k})>=((\mathrm{RRV} 1+\mathrm{RRV} 2+\mathrm{RRV} 3) / 3 * \mathrm{ra}$ mppercent) \&\& (k-

lastR $)>((($ RRT1+RRT2+RRT3)/3)*blankper cent)

$\operatorname{trigger}(\mathrm{k})=1$;

if $(\mathrm{k}-$

lastR $)>(($ RRT1+RRT2+RRT3)/3 $) *(1+$ RRTV ariabilityfactor) $\|$ (k-

lastR $)<((\mathrm{RRT} 1+\mathrm{RRT} 2+\mathrm{RRT} 3) / 3) *(1-$

RRTvariabilityfactor)

$\operatorname{pstats}(1: 4, \mathrm{pz})=0$;

tstats $(1: 4, \mathrm{tz})=0$;

$\operatorname{rstats}(1: 4, \mathrm{rz})=0$;

$\mathrm{rz}=\mathrm{rZ}+1$;

$\mathrm{pz}=\mathrm{pz}+1$;

$\mathrm{tz}=\mathrm{tz}+1$;

else

RRT3=RRT2;

RRT2=RRT1;

RRT1=k-lastR;

indexing

$\%$ p,t,r wave capture and voltage

pwave(round(k-(k-

lastR)*(ppercent+rpercentbackward)):round(

$\mathrm{k}-(\mathrm{k}-$

lastR $)^{*}$ rpercentbackward $\left.)\right)=E C G($ round $(k-$

(k-

lastR)*(ppercent+rpercentbackward)):round( $\mathrm{k}-(\mathrm{k}-\mathrm{lastR}) *$ rpercentbackward));

pstats $(1, \mathrm{pz})=\mathrm{abs}(\max ($ pwave $(\operatorname{round}(\mathrm{k}-(\mathrm{k}-$ lastR)*(ppercent+rpercentbackward)):round( $\mathrm{k}$-(k-lastR)*rpercentbackward $))$ )$\min$ (pwave(round(k-(k-
lastR)*(ppercent+rpercentbackward)):round( k-(k-lastR)*rpercentbackward)))); twave(round(k-(k-lastR)*(1rpercentforward)):round(k-(k-lastR)*(1rpercentforward-tpercent $))=E C G(\operatorname{round}(k-$ (k-lastR)*(1-rpercentforward)):round(k-(klastR)*(1-rpercentforward-tpercent)));

$\operatorname{tstats}(1, \mathrm{tz})=\operatorname{abs}(\max ($ twave $(\operatorname{round}(\mathrm{k}-(\mathrm{k}-$ lastR)*(1-rpercentforward)):round(k-(klastR $)^{*}(1$-rpercentforward-tpercent $\left.\left.)\right)\right)$ min(twave(round(k-(k-lastR)*(1rpercentforward)):round(k-(k-lastR)*(1rpercentforward-tpercent))))); rwave(round $(\mathrm{k}-(\mathrm{k}-$

lastR)*(rpercentbackward $))$ :round(k+(klastR $) *(\operatorname{rpercentforward})))=\mathrm{ECG}(\operatorname{round}(\mathrm{k}-$ (k-lastR)*(rpercentbackward)):round(k+(klastR)*(rpercentforward)));

rstats $(1, \mathrm{rz})=\mathrm{abs}(\max ($ rwave $($ round $(\mathrm{k}-(\mathrm{k}-$ lastR)*(rpercentbackward $))$ :round $(\mathrm{k}+(\mathrm{k}-$ lastR)*(rpercentforward))))$\min ($ rwave(round(k-(klastR $)^{*}($ rpercentbackward $\left.)\right)$ :round(k+(klastR)*(rpercentforward))))); $\%$ p,t wave / $\mathrm{r}$ wave ratio indexing

pstats $(2, \mathrm{pz})=\operatorname{pstats}(1, \mathrm{pz}) / \mathrm{rstats}(1, \mathrm{rz})$; $\operatorname{tstats}(2, \mathrm{tz})=\operatorname{tstats}(1, \mathrm{tz}) / \mathrm{rstats}(1, \mathrm{rz})$; $[\operatorname{maxv}, \operatorname{prt} 1]=\max (\operatorname{rwave}(\operatorname{round}(\mathrm{k}-$

(k-lastR)*(rpercentbackward)):round $(\mathrm{k}+(\mathrm{k}-$ lastR)*(rpercentforward)))); if ipwave $==1$

$[\mathrm{maxv}, \operatorname{prt} 2]=\min ((\mathrm{pwave})($ round $(\mathrm{k}-(\mathrm{k}-$ lastR)*(ppercent+rpercentbackward)):round( k-(k-lastR)*rpercentbackward)))); else

[maxv,prt2] $=\max (($ pwave $($ round $(\mathrm{k}-(\mathrm{k}-$ lastR)*(ppercent+rpercentbackward)):round( k-(k-lastR)*rpercentbackward)))); end $\operatorname{pstats}(3, \mathrm{pz})=(\mathrm{prt} 1+\mathrm{round}(\mathrm{k}-(\mathrm{k}-$ lastR)*(rpercentbackward))-prt2-round(k-(klastR)*(ppercent+rpercentbackward)))/400; if $\mathrm{pz}>1$; if $\operatorname{pstats}(1: 4, \mathrm{pz}-1)==0$

pstats $(4, \mathrm{pz})=(\operatorname{prt} 2+\operatorname{round}(\mathrm{k}-(\mathrm{k}-$

lastR)*(ppercent+rpercentbackward)) $) / 400$; psave $=$ pstats $(4, p z)$; else $\operatorname{pstats}(4, \mathrm{pz})=(\operatorname{prt} 2+\operatorname{round}(\mathrm{k}-$

$(\mathrm{k}-$ 
lastR $) *($ ppercent+rpercentbackward)))/400psave;

psave $=(\operatorname{prt} 2+$ round $(\mathrm{k}-(\mathrm{k}-$

lastR)*(ppercent+rpercentbackward)))/400; end

else

pstats $(4, \mathrm{pz})=(\operatorname{prt} 2+\operatorname{round}(\mathrm{k}-(\mathrm{k}-$

lastR)*(ppercent+rpercentbackward $))) / 400$; psave $=$ pstats $(4, p z)$;

end

if itwave $==1$

$[$ maxv,trt2] $=\min (($ twave $($ round $(\mathrm{k}-(\mathrm{k}-$

lastR)*(1-rpercentforward)):round(k-(k-

lastR)*(1-rpercentforward-tpercent))))); else

[maxv,trt2] $=\max (($ twave $($ round $(\mathrm{k}-(\mathrm{k}-$ lastR)*(1-rpercentforward)):round(k-(k-

lastR)*(1-rpercentforward-tpercent))))); end tstats $(3, \mathrm{tz})=(\operatorname{trt} 2+\operatorname{round}(\mathrm{k}-(\mathrm{k}-$

lastR)*(1-rpercentforward))-lastR)/400; if $\mathrm{tz}>1$;

if $\operatorname{tstats}(1: 4, \mathrm{tz}-1)==0$

tstats $(4, \mathrm{tz})=(\operatorname{trt} 2+\operatorname{round}(\mathrm{k}-(\mathrm{k}-\mathrm{lastR}) *(1-$ rpercentforward)) $) / 400$;

tsave $=$ tstats $(4, \mathrm{tz})$;

else tstats $(4, \mathrm{tz})=(\operatorname{trt} 2+\operatorname{round}(\mathrm{k}-(\mathrm{k}-$

lastR)*(1-rpercentforward)))/400-tsave; tsave $=(\operatorname{trt} 2+$ round $(k-(k-$

lastR)*(1-rpercentforward)))/400;

end

else

tstats $(4, \mathrm{tz})=(\operatorname{trt} 2+$ round $(\mathrm{k}-(\mathrm{k}-$

lastR)*(1-rpercentforward)))/400;

tsave $=$ tstats $(4, \mathrm{tz})$;

end

$\%$ qrslength routine rmstart=round $(\mathrm{k}-(\mathrm{k}-$

lastR)*(rpercentbackward)); rmend $=\operatorname{round}(\mathrm{k}+(\mathrm{k}-$

lastR)*(rpercentforward)); qrsseg $=\mathrm{ECG}(1$, rmstart:rmend $)$; qrsseg $=$ qrsseg $+(0-\min ($ qrsseg $))$; qrsseg=qrsseg/max(qrsseg); [qrsmax

ignoreindex $]=\max ($ qrsseg $)$; averaging $=6$; qrswidthstart $=0$; qrsdiffpercent=.8; for $\mathrm{jkl}=$ averaging:-1:1 clear averagingm iii $=0$; if qrswidthstart $==0$

for $\mathrm{ii}=($ ignoreindex $):-1: 1$;

if qrswidthstart $==0$

if $\mathrm{iii}<\mathrm{jkl}$

averagingm $(\mathrm{iii}+1)=$ qrsseg $(\mathrm{ii})$; iii=iii+1;

else

for

$11=1: 1:($ length(averagingm) -1$)$

averagingm $(11)=$ averagingm $(11+1)$;

end

averagingm $(\mathrm{jkl})=\mathrm{qrsseg}(\mathrm{ii})$;

if (qrsmax-

mean $($ averagingm $))>=$ qrsdiffpercent $*$ (qrsma

$\mathrm{x}-\min (\mathrm{qrsseg}(1$ :ignoreindex $))$ ) qrswidthstart=ii;

$$
\text { end }
$$

end

end

end

end

averaging=averaging -1 ;

end

averaging $=6$;

qrswidthend $=0$;

for $\mathrm{jkl}=$ averaging:-1:1

clear averagingm

iii $=0$;

if qrswidthend $==0$

for

$\mathrm{ii}=($ ignoreindex $): 1:$ length (qrsseg);

if qrswidthend $==0$

if $\mathrm{iii}<\mathrm{jkl}$

averagingm $(\mathrm{iii}+1)=\mathrm{qrsseg}(\mathrm{ii})$; iii= $=$ iii +1 ;

else

for

$11=1: 1:($ length(averagingm)-1)

averagingm $(11)=$ averagingm $(11+1)$;

end averagingm $(\mathrm{jkl})=\mathrm{qrsseg}(\mathrm{ii})$; if (qrsmax-

mean (averagingm $))>=$ qrsdiffpercent $*$ (qrsma

$\mathrm{x}-\min ($ qrsseg(ignoreindex:end))

qrswidthend=ii; end

end

end

end

end

averaging=averaging -1 ;

end 


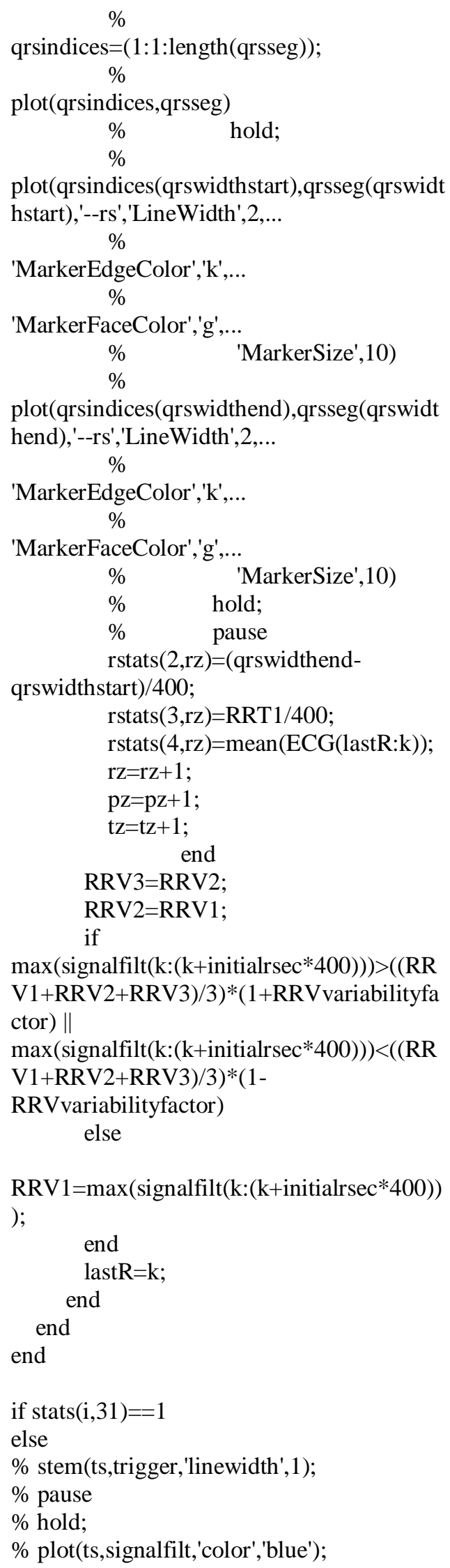

$\%$

qrsindices $=(1: 1:$ length $($ qrsseg $))$; $\%$

plot(qrsindices,qrsseg) $\%$ hold;

$\%$

plot(qrsindices(qrswidthstart), qrsseg(qrswidt hstart),'--rs','LineWidth',2,...

$\%$

'MarkerEdgeColor','k',...

$\%$

'MarkerFaceColor','g',... $\% \quad$ 'MarkerSize',10) $\%$

plot(qrsindices(qrswidthend), qrsseg(qrswidt hend), '--rs','LineWidth',2,... $\%$

'MarkerEdgeColor','k',... $\%$

'MarkerFaceColor','g',...

$\begin{array}{ll}\% & \text { 'MarkerSize',10) } \\ \% & \text { hold; } \\ \% & \text { pause } \\ \text { rstats(2,rz)=(qrswidthend- }\end{array}$

qrswidthstart)/400;

$\operatorname{rstats}(3, \mathrm{rz})=\mathrm{RRT} 1 / 400$;

rstats $(4, \mathrm{rz})=\operatorname{mean}(\mathrm{ECG}(\mathrm{lastR}: \mathrm{k}))$;

$\mathrm{rz}=\mathrm{rz}+1$;

$\mathrm{pz}=\mathrm{pz}+1$;

$\mathrm{tz}=\mathrm{tz}+1$

end

RRV3=RRV2;

RRV2=RRV1;

if

$\max ($ signalfilt $(\mathrm{k}:(\mathrm{k}+$ initialrsec $* 400)))>((\mathrm{RR}$

V1+RRV2+RRV3)/3)*(1+RRVvariabilityfa ctor) $\|$

$\max ($ signalfilt $(\mathrm{k}:(\mathrm{k}+$ initialrsec $* 400)))<((\mathrm{RR}$

V1+RRV2+RRV3)/3)*(1-

RRVvariabilityfactor)

else

RRV1 $=\max ($ signalfilt $(\mathrm{k}:(\mathrm{k}+$ initialrsec $* 400))$

) ;

end

lastR=k;

end

end

end

if $\operatorname{stats}(\mathrm{i}, 31)==1$

else

$\%$ stem(ts,trigger,'linewidth',1);

$\%$ pause

$\%$ hold;

$\%$ plot(ts,signalfilt,'color','blue');

$\%$ plot(ts,trigger,'linewidth',2,'color','red');

$\% \operatorname{plot}(\mathrm{t},-$

1 *screwd,'linewidth',2,'color','magenta');

$\%$ hold;

$\%$ for $1=0: 3: 27$

$\% \quad x \lim ([1(1+3)])$

$\%$ pause

$\%$ end

end

$\%$

plot(ts,ECG(:),'color','black','linewidth',1);

$\%$ hold;

$\%$ plot(ts,pwave,'color','red','linewidth',2)

$\%$ plot(ts,twave,'color','blue','linewidth',2)

$\%$ plot(ts,rwave,'color','green','linewidth',2)

$\%$ for $\mathrm{l}=0: 3: 27$

$\% \quad x \lim ([1(1+3)])$

$\%$ pause

$\%$ end

$\%$ hold;

subq3d=trigger;

save(sprintf('\%s\%s\%s\%i\%s',cow,',','PQRST ',i-

1,'_3.mat'),'pstats','rstats','tstats','pwave','twa ve','rwave');

disp('PQRST Matrices saved')

$\%$ Step-through Trigger Analysis

truepositive $=0$

falsepositive $=0$;

falsenegative $=0$;

\% Truepositive and Falsenegative Search

for $\mathrm{j}=1: 1$ :(length(subq3d)-resolution)

if $\operatorname{screwd}(1, \mathrm{j})==1$

bug1=(j-resolution $)$;

if $(\mathrm{j}$-resolution $)<=0$

bug $1=1$;

end

if

$\operatorname{sum}(\operatorname{subq} 3 \mathrm{~d}(1, \operatorname{bug} 1: 1: j+$ resolution $))==1$ truepositive $=$ truepositive +1 ; elseif

$\operatorname{sum}(\operatorname{subq} 3 \mathrm{~d}(1, \operatorname{bug} 1: 1: \mathrm{j}+$ resolution $))==0$ falsenegative $=$ falsenegative +1 ;

else

disp('Triggering error noted, check

resolution vs blanking period') end

end

end

$\%$ Falsepositive Search

for $\mathrm{j}=1: 1$ :(length(subq3d)-resolution) 


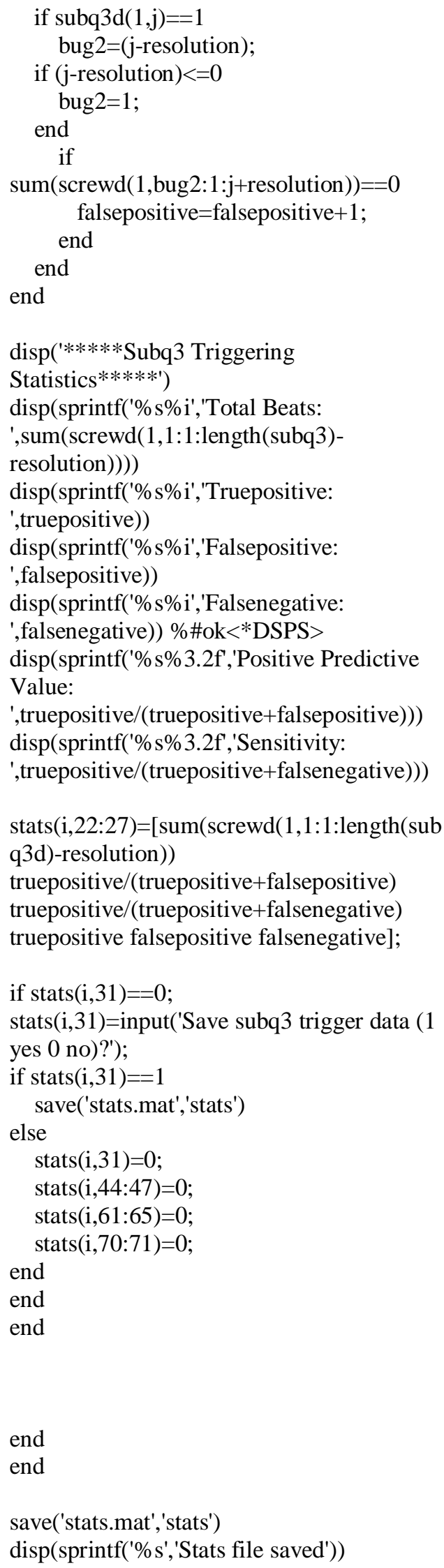

\section{B. Data Processing Code}

clc

clear all

ampfactor $=10$; cow='J071';

load('stats.mat'); master $1=$ zeros(length(stats),15); master2=zeros(length(stats),15); master3=zeros(length(stats),15);

i $1=1$;

i2 $=1$;

i3 $=1$;

ip1=1;

ip2=1;

ip3 $=1$;

it $1=1$;

it $2=1$;

it $3=1$;

ir $1=1$;

ir $2=1$;

ir $3=1$;

for $\mathrm{i}=1: 1$ :(length(stats))

$\%$ Subq1

\%main statistics

if $\operatorname{stats}(i, 1)==1$ triggerstats $1(\mathrm{i} 1,1: 3)=\operatorname{stats}(\mathrm{i}, 10: 12)$; $\mathrm{i} 1=\mathrm{i} 1+1$; master $1(\mathrm{i}, 1: 3)=\operatorname{stats}(\mathrm{i}, 10: 12)$;

\%PQRS statistics

\%PSTATS

if $\operatorname{stats}(i, 2)==1$

call=sprintf('\%s\%s\%s\%i\%s',cow,'_,'PQRST_,'i1,'_1.mat');

clear pstats

clear pwave

clear rstats

clear rwave

clear tstats

clear twave 


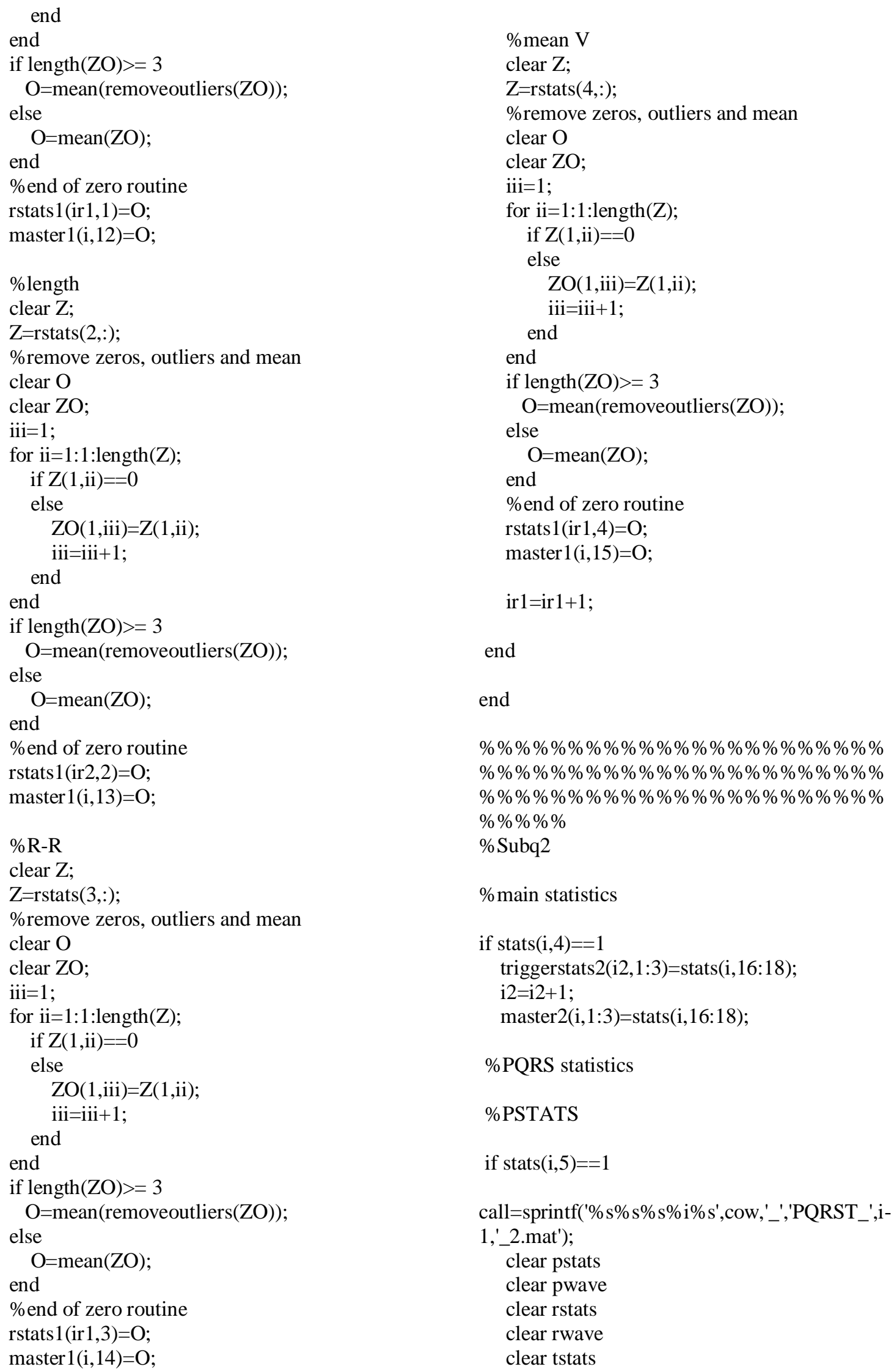




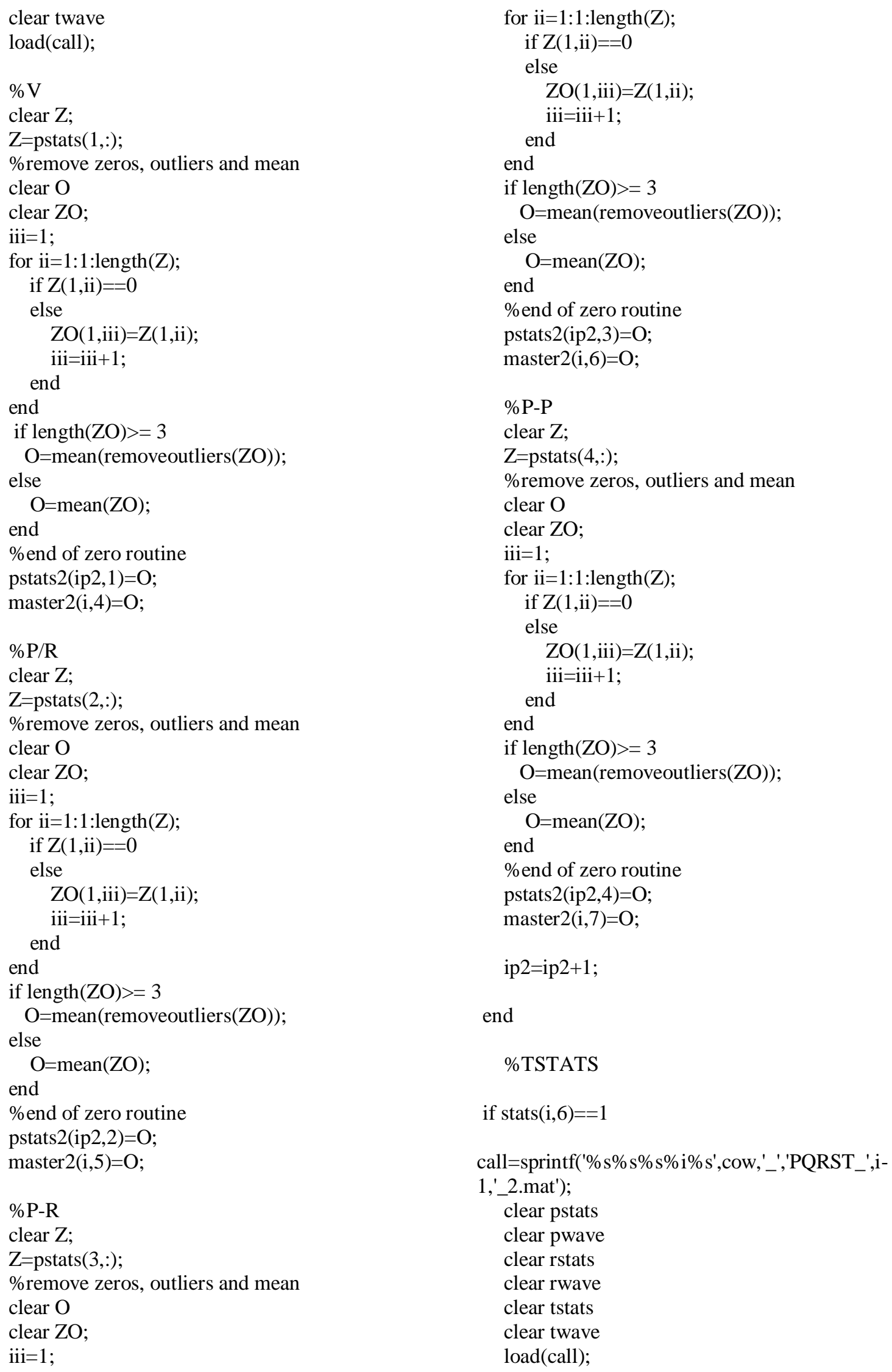

end

\section{\%TSTATS}

if $\operatorname{stats}(i, 6)==1$

call=sprintf('\%s\%s\%s\%i\%s',cow,',','PQRST_,'i1,'_2.mat');

clear pstats

clear pwave

clear rstats

clear rwave

clear tstats

clear twave

load(call); 


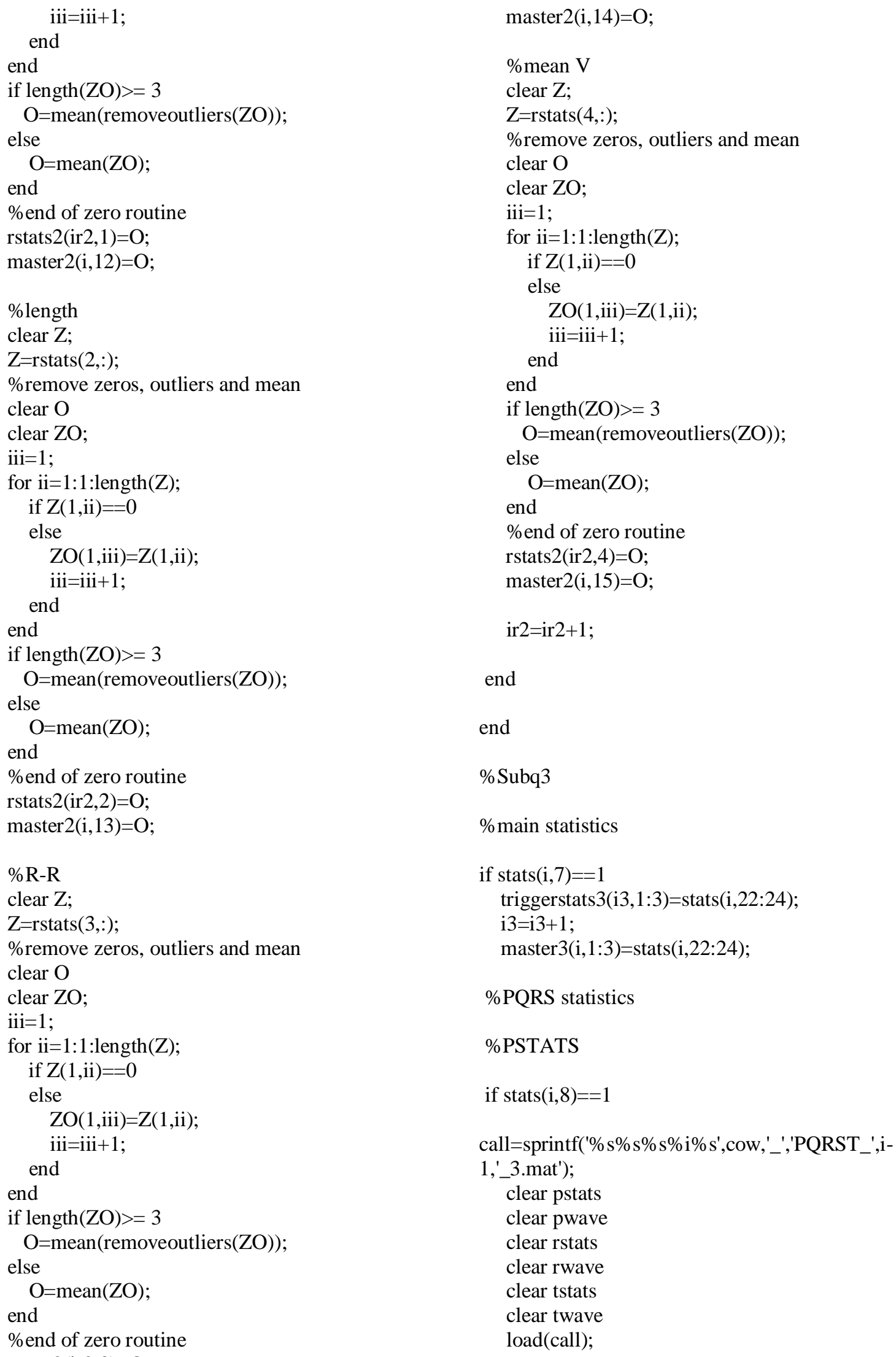




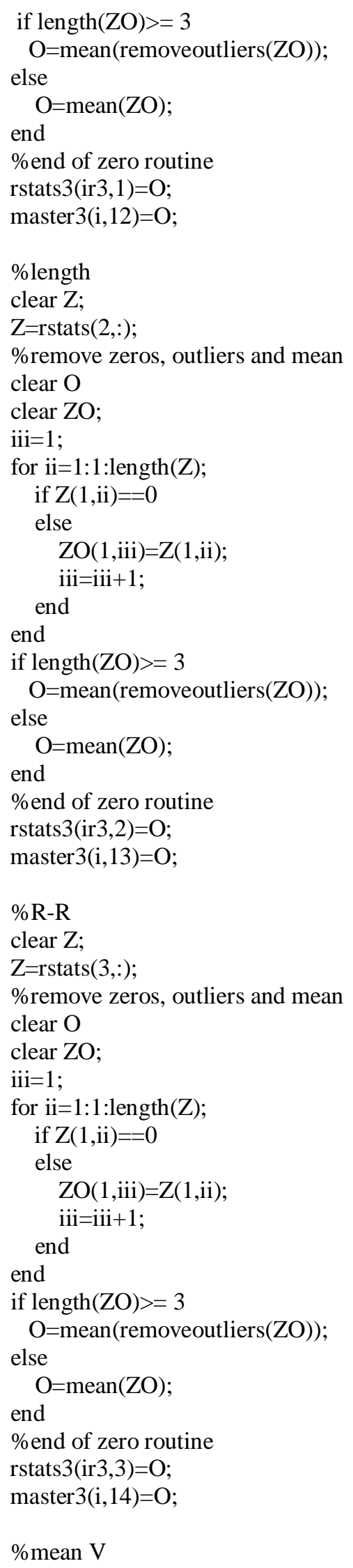




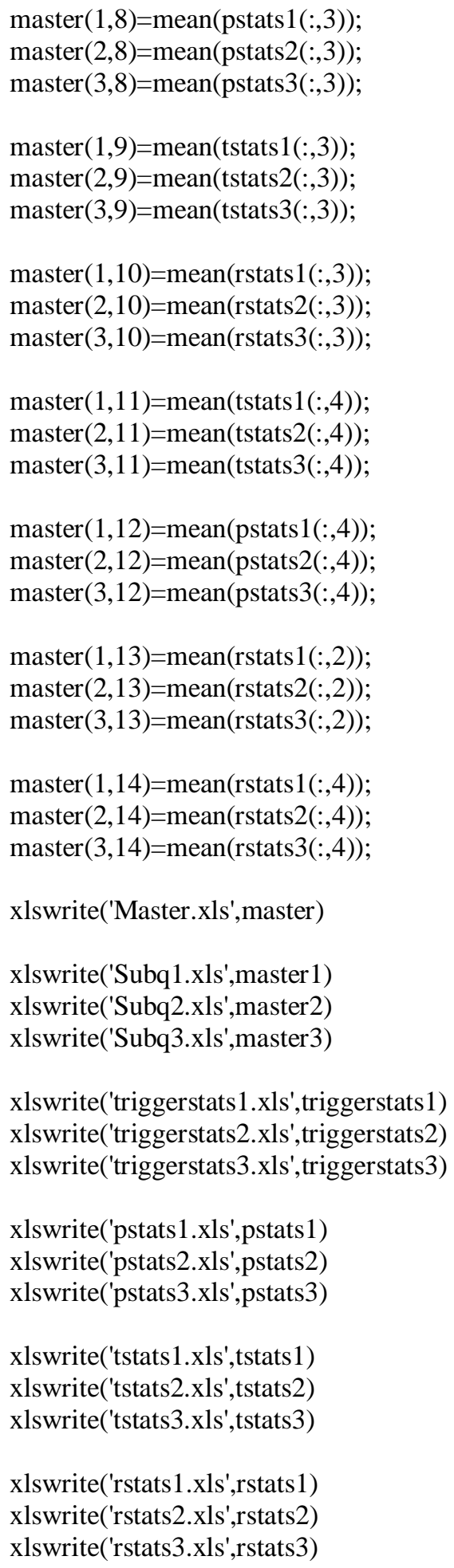

disp('Data compiled, excel files written') 


\section{Miscellaneous Code}

\section{ECG Extract}

\%ECG Data Extraction Program

\%Sean Warren

$\%$ Ver $7 / 14 / 2010$

clear all

clc

\%Load Dir

$\operatorname{dir}=\operatorname{dir}(' *$ dat');

$\%$ Data Load Parameters

$\%$ Change these values to change how the program takes the data

Cow=sprintf('\%s','J066'); $\quad$ \%Change this to Cow \#

Tprompt=0; $\quad$ \%Change this to 1 if you want prog to prompt for time of data set. time $=30 ; \quad \%$ Time if $\mathrm{Tprompt}=0$ Hzprompt $=0 ; \quad$ \%Change this to 1 if you want prog to prompt for $\mathrm{Hz}$ of data set. $\mathrm{hz}=400 ; \quad \% \mathrm{hz}$ if Hzprompt=1 counter $=0$;

for $\mathrm{i}=1: 1:$ length(dir')

dataascii=importdata(dir(i).name); data=dataascii.data;

\% Prompts for Channels and Time

$\%$ screwC=input('Screw-in Channel:');

$\%$ subq1C=input('SubQ 1 Channel:');

$\%$ subq2C=input('SubQ 2 Channel:');

$\%$ subq3C=input('SubQ 3 Channel:');

screwC $=4$;

subq $1 \mathrm{C}=2$;

subq2C $=5$;

subq $3 \mathrm{C}=3$;

if Tprompt==1;

time=input('Time of dataset:')

end

if Hzprompt==1; hz=input('Hz of dataset:')

end

\%Saving Data $\mathrm{t}=[0: 1 / \mathrm{hz:time}-1 / \mathrm{hz}]$;

subq1=data(:,subq1C)';

subq2=data(:,subq2C)';

subq3=data(:,subq3C)';

screw=data $(: \text { screwC })^{\prime}$;

$\% \operatorname{plot}(\mathrm{t}, \mathrm{screw}, \mathrm{t}$, subq1,t,subq2,t,subq3)

$\% x \lim ([05])$;

$\%$ legend('screw','subq1','subq2','subq3');

$\%$ pause

filename=sprintf('\%s\%c\%i\%s',Cow,',',count er,'.mat');

save(filename,'subq1','subq2','subq3','screw',' t')

counter=counter +1

end

$\%$ filenamevar5=sprintf('\%i',VBPM);

$\%$

filename $1=\operatorname{char}(\operatorname{strcat}(\{$ 'pressures' $\},\{$ 'VADp $\left.\mathrm{w}^{\prime}\right\},\{$ filenamevar3 $\},\{$ 'ts' $\},\{$ filenamevar2 $\},\{$ 's $\left.\mathrm{pt}^{\prime}\right\},\{$ filenamevar4 $\},\{$ 'VBPM' $\},\{$ filenamevar $5\})$ );

\section{"Play Around" Script}

clear all

clc

load('ECG.mat');

$\mathrm{t}=0: 1 / 400$ :(length(ECG)-1)/400;

hold off

for $\mathrm{i}=1: 1:$ length(ECG);

$\mathrm{i}=1$;

clear ecg

$\%$ FFT

$\%$ fs $=400$

$\% \mathrm{~L}=$ length(ECG);

$\%$ NFFT $=2^{\wedge}$ nextpow2(L); \% Next power

of 2 from length of $y$

$\% \mathrm{Y}=\mathrm{fft}(\mathrm{ECG}(1,:), \mathrm{NFFT}) / \mathrm{L}$;

$\% \mathrm{f}=\mathrm{fs} / 2 * \operatorname{linspace}(0,1, \mathrm{NFFT} / 2+1)$;

$\%$ figure

$\% \operatorname{plot}(\mathrm{f}, 2 * \operatorname{abs}(\mathrm{Y}(1: \mathrm{NFFT} / 2+1)))$

$\%$ title('Single-Sided Amplitude Spectrum

of $\left.\mathrm{y}(\mathrm{t})^{\prime}\right)$

$\%$ xlabel('Frequency $\left.(\mathrm{Hz})^{\prime}\right)$

$\%$ ylabel('|Y(f)|')

$\% x \lim \left(\left[\begin{array}{ll}0 & 100]\end{array}\right)\right.$

$\%$ pause

$\%$

$\%[\mathrm{fft}, \mathrm{f}]=$ positiveFFT(ECG(i,:),400); 
RRV1=max (signalfilt $(\mathrm{k}:(\mathrm{k}+$ initialrsec*400))

);

initial $R=$ initial $R+1$;

RRT3=RRT2-RRT3;

RRT2=RRT1-RRT2;

RRT1=RRT2;

end

elseif initialR $==2$

if signalfilt $(\mathrm{k})>=\mathrm{ithresh} \& \&(\mathrm{k}-$

RRT1)>iblank

trigger $(\mathrm{k})=1$

RRT3=RRT2;

RRT2=RRT1;

RRT1=k;

RRV3=RRV2;

RRV2=RRV1;

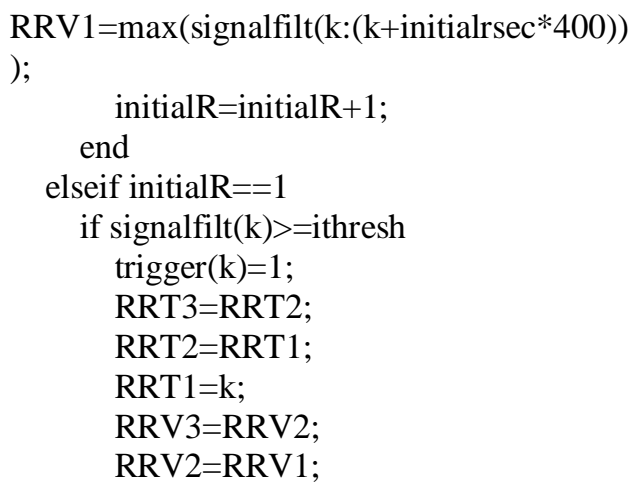

RRV1=max (signalfilt $(\mathrm{k}:(\mathrm{k}+$ initialrsec*400) $)$

);

initialR=initialR+1;

end

else

if

signalfilt $(\mathrm{k})>=((\mathrm{RRV} 1+\mathrm{RRV} 2+\mathrm{RRV} 3) / 3 * \mathrm{ra}$ mppercent) \&\& (k-

lastR $)>(((\mathrm{RRT} 1+\mathrm{RRT} 2+\mathrm{RRT} 3) / 3) *$ blankper cent)

trigger $(\mathrm{k})=1$

RRT3=RRT2;

RRT2=RRT1;

if $(\mathrm{k}-$

lastR $)>((\mathrm{RRT} 1+\mathrm{RRT} 2+\mathrm{RRT} 3) / 3) *(1+\mathrm{RRTv}$

ariabilityfactor) $\|(\mathrm{k}-$

lastR $)<((\mathrm{RRT} 1+\mathrm{RRT} 2+\mathrm{RRT} 3) / 3) *(1-$

RRTvariabilityfactor)

else

RRT1=k-lastR;

$\%$ p,t,r wave capture and voltage

indexing

pwave(round $(\mathrm{k}-(\mathrm{k}-$

lastR)*(ppercent+rpercentbackward)):round( $\mathrm{k}-(\mathrm{k}-$
lastR)*rpercentbackward $))=E C G(\mathrm{i}$, round $(\mathrm{k}-$

(k-

lastR)*(ppercent+rpercentbackward)):round( k-(k-lastR)*rpercentbackward));

pstats $(1, \mathrm{pz})=\mathrm{abs}(\max ($ pwave $($ round $(\mathrm{k}-(\mathrm{k}-$ lastR)*(ppercent+rpercentbackward)):round( $\mathrm{k}$-(k-lastR)*rpercentbackward)))-

$\min$ (pwave)(round(k-(k-

lastR)*(ppercent+rpercentbackward)):round( k-(k-lastR)*rpercentbackward)))); twave(round(k-(k-lastR)*(1rpercentforward)):round(k-(k-lastR)*(1rpercentforward-tpercent $))=E C G(i$, round $(k-$ (k-lastR)*(1-rpercentforward)):round(k-(klastR)*(1-rpercentforward-tpercent)));

tstats $(1, \mathrm{tz})=\mathrm{abs}(\max (\mathrm{twave})$ round $(\mathrm{k}-(\mathrm{k}-$ lastR)*(1-rpercentforward)):round(k-(klastR)*(1-rpercentforward-tpercent))))min (twave(round(k-(k-lastR)*(1rpercentforward)):round(k-(k-lastR)*(1rpercentforward-tpercent))))); rwave(round $(\mathrm{k}-(\mathrm{k}-$

lastR)*(rpercentbackward)):round(k+(klastR)*(rpercentforward $)))=E C G(i$, round $(k-$ (k-lastR)*(rpercentbackward)):round(k+(klastR)*(rpercentforward)));

$\operatorname{rstats}(1, \mathrm{rz})=\mathrm{abs}(\max (\mathrm{rwave})($ round $(\mathrm{k}-(\mathrm{k}-$ lastR)*(rpercentbackward)):round(k+(klastR)*(rpercentforward))))$\min$ (rwave(round(k-(k-

lastR $) *($ rpercentbackward $))$ :round $(\mathrm{k}+(\mathrm{k}-$

lastR)*(rpercentforward $))))$ ); $\% \mathrm{p}, \mathrm{t}$ wave / $\mathrm{r}$ wave ratio indexing

pstats $(2, \mathrm{pz})=\mathrm{pstats}(1, \mathrm{pz}) / \mathrm{rstats}(1, \mathrm{rz})$; $\operatorname{tstats}(2, \mathrm{tz})=\operatorname{tstats}(1, \mathrm{tz}) / \mathrm{rstats}(1, \mathrm{rz})$; [maxv, prt1] $=\max ($ rwave $($ round $(\mathrm{k}-$

(k-lastR)*(rpercentbackward $))$ :round $(\mathrm{k}+(\mathrm{k}-$ lastR)*(rpercentforward)))); \%qrslength routine rmstart=round $(\mathrm{k}-(\mathrm{k}-$

lastR)*(rpercentbackward)); rmend=round $(\mathrm{k}+(\mathrm{k}-$

lastR)*(rpercentforward)); qrsseg=ECG(i,rmstart:rmend); qrsseg $=$ qrsseg $+(0-\min ($ qrsseg $))$; qrsseg=qrsseg $/$ max (qrsseg); [qrsmax

ignoreindex]=max(qrsseg); averaging $=6$; clear averagingm qrswidthstart=0; iii $=0$; 
qrsdiffpercent=.8;

for $\mathrm{jkl}=$ averaging:- $1: 1$

clear averagingm

iii $=0$;

if qrswidthstart $==0$

for ii=(ignoreindex):-1:1;

if qrswidthstart $==0$

if iii<jkl

averagingm(iii +1$)=q r s s e g(i i)$; iii=iii +1 ;

else

for

$11=1: 1:($ length(averagingm) -1$)$

averagingm $(11)=$ averagingm $(11+1)$;

end

averagingm $(\mathrm{jkl})=\mathrm{qrsseg}(\mathrm{ii})$; if (qrsmax-

mean(averagingm) $)>=$ qrsdiffpercent $*$ (qrsma

$\mathrm{x}$-min(qrsseg(1:ignoreindex $))$ )

qrswidthstart=ii;

$$
\text { end }
$$

end

end

end

end

averaging=averaging-1;

end

averaging $=6$;

qrswidthend $=0$;

for $\mathrm{jkl}=$ averaging:-1:1

clear averagingm

iii $=0$;

if qrswidthend $==0$

for

ii=(ignoreindex):1:length(qrsseg);

if qrswidthend $==0$

if $\mathrm{iii}<\mathrm{jkl}$

averagingm(iii +1$)=q \operatorname{rsseg}(\mathrm{ii})$; iii=iii+1;

else

for

$11=1: 1$ (length(averagingm)-1)

averagingm(ll)=averagingm(ll+1);

end

averagingm(jkl)=qrsseg(ii);

if (qrsmax-

mean (averagingm $))>=q r$ sdiffpercent $*($ qrsma

$\mathrm{x}$-min(qrsseg(ignoreindex:end))$$
\text { end }
$$

end

end

end end

averaging=averaging- 1 ;

end

$\% \quad$ qrsindices $=(1: 1:$ length(qrsseg) $)$;

$\% \quad$ plot(qrsindices,qrsseg)

$\%$ hold;

$\%$

plot(qrsindices(qrswidthstart),qrsseg(qrswidt hstart),'--rs','LineWidth',2,...

$\% \quad$ 'MarkerEdgeColor','k',,...

$\% \quad$ 'MarkerFaceColor','g',,...

$\%$ 'MarkerSize',10)

$\%$

plot(qrsindices(qrswidthend), qrsseg(qrswidt hend),'--rs','LineWidth',2,...

$\% \quad$ 'MarkerEdgeColor','k',,...

$\% \quad$ 'MarkerFaceColor','g',,...

$\%$ 'MarkerSize',10)

$\%$ hold;

$\% \quad$ pause

if ipwave $==1$

[maxv,prt2] $=\min (($ pwave (round $(\mathrm{k}-(\mathrm{k}-$

lastR)*(ppercent+rpercentbackward)):round(

k-(k-lastR)*rpercentbackward)))); else

[maxv,prt2] $=\max (($ pwave (round $(\mathrm{k}-(\mathrm{k}-$

lastR)*(ppercent+rpercentbackward)):round(

k-(k-lastR)*rpercentbackward)))); end

$\%$

[maxv,prt2] $=\min (\operatorname{abs}(\operatorname{diff}($ pwave $($ round $(\mathrm{k}-$

(k-

lastR)*(ppercent+rpercentbackward)):round(

$\mathrm{k}$-(k-lastR)*rpercentbackward))))); pstats $(3, \mathrm{pz})=($ prt $1+\operatorname{round}(\mathrm{k}-(\mathrm{k}-$

lastR)*(rpercentbackward))-prt2-round(k-(klastR)*(ppercent+rpercentbackward)))/400; if itwave $==1$

[maxv,trt2] $=\min (($ twave $($ round $(\mathrm{k}-(\mathrm{k}-$

lastR)*(1-rpercentforward)):round(k-(k-

lastR)*(1-rpercentforward-tpercent))))); else

$[\operatorname{maxv}, \operatorname{trt} 2]=\max ((\mathrm{twave})($ round $(\mathrm{k}-(\mathrm{k}-$

lastR)*(1-rpercentforward)):round(k-(k-

lastR)*(1-rpercentforward-tpercent))))); end

$\%$

[maxv,trt2] $=\max (\operatorname{abs}(\operatorname{diff}($ twave $($ round $(\mathrm{k}-(\mathrm{k}-$ 


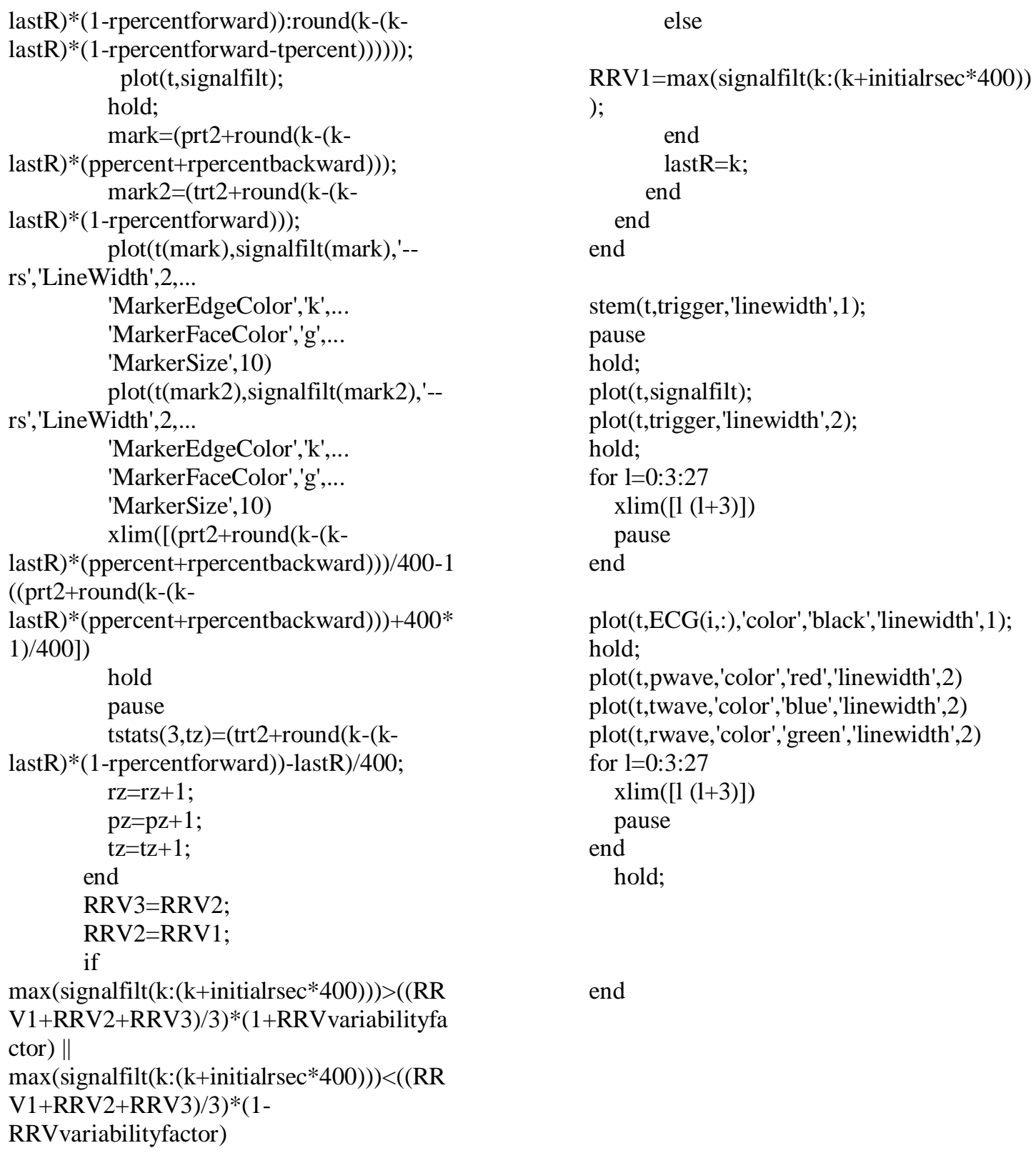

end 